International Journal of Algebra and Computation Vol. 16, No. 4 (2006) 689-737

(C) World Scientific Publishing Company

\title{
FULLY RESIDUALLY FREE GROUPS AND GRAPHS LABELED BY INFINITE WORDS
}

\author{
ALEXEI G. MYASNIKOV \\ McGill University, Department of Mathematics and Statistics \\ 805 Sherbrooke W., Montreal QC H3A 2K6, Canada \\ alexeim@att.net \\ VLADIMIR N. REMESLENNIKOV \\ Omsk Branch of the Mathematical Institute SB RAS \\ 13 Pevtsova Street, 644099, Omsk, Russia \\ remesl@itam.omsk.net.ru \\ DENIS E. SERBIN \\ McGill University, Department of Mathematics and Statistics \\ 805 Sherbrooke W., Montreal QC H3A 2K6, Canada \\ zloidyadya@yahoo.com \\ Received 15 October 2003 \\ Revised 13 May 2005 \\ Communicated by O. Kharlampovich
}

\begin{abstract}
Let $F=F(X)$ be a free group with basis $X$ and $\mathbb{Z}[t]$ be a ring of polynomials with integer coefficients in $t$. In this paper we develop a theory of $(\mathbb{Z}[t], X)$-graphs - a powerful tool in studying finitely generated fully residually free (limit) groups. This theory is based on the Kharlampovich-Myasnikov characterization of finitely generated fully residually free groups as subgroups of the Lyndon's group $F^{\mathbb{Z}[t]}$, the author's representation of elements of $F^{\mathbb{Z}[t]}$ by infinite $(\mathbb{Z}[t], X)$-words, and Stallings folding method for subgroups of free groups. As an application, we solve the membership problem for finitely generated subgroups of $F^{\mathbb{Z}[t]}$, as well as for finitely generated fully residually free groups.
\end{abstract}

Keywords: Fully residually free groups; folded graphs, membership problem; infinite words.

Mathematics Subject Classification 2000: 22E46, 53C35, 57S20

\section{Introduction}

In the seminal paper [12] Stallings introduced an extremely useful notion of a folding of graphs and initiated the study of subgroups (and automorphisms) of free groups via folded directed labeled graphs. This approach turned out to be very influential and allowed researchers to prove many new results and simplify old proofs. We refer 
to a survey [4] for a detailed discussion of the known results and methods in this area. Here we would like only to mention that, among other things, the Stallings technique gives a very fast algorithm for the Uniform Membership Problem in free groups. Namely, given a tuple of words $h_{1}, \ldots, h_{n}$ of a free group $F(X)$ with basis $X$ one can construct in at most quadratic time in $\left|h_{1}\right|+\cdots+\left|h_{n}\right|$ the Stallings folding (viewed as a deterministic finite automata) $\Gamma_{H}$ which accepts precisely the reduced words from $F(X)$ which belong to the subgroup $H=\left\langle h_{1}, \ldots, h_{n}\right\rangle$. Now, given a reduced word $w \in F(X)$ one can check, in linear time in $|w|$, whether $w \in H$ or not. One of the crucial features of the Stallings method is that the graph $\Gamma(H)$ carries all the essential information about the subgroup $H$ itself.

Observe that free groups are precisely the groups that admit free actions on simplicial trees. In the paper [13] Stallings offered a way to extend his ideas to non-free actions of groups on graphs and trees. Bestvina and Feighn [2] and, independently, Dunwoody [3] gave a systematic treatment of Stalling's method in the context of graphs of groups and groups acting on simplicial trees. A more geometric and unified approach to this topic which relies on Bass-Serre theory $[11,1]$ as well as the foldings technique of Stallings-Bestvina-Feighn-Dunwoody was recently developed in [5].

Research on the membership problem in amalgamated free products, HNNextensions and more generally, fundamental groups of graphs of groups has a long and rich history. In 1958 Mihailova [8] constructed a remarkable example of a finitely generated subgroup of the direct product $F_{2} \times F_{2}$ of two free groups of rank two with unsolvable membership problem. Most of the early results used normal forms techniques, so the proofs were very technical and sometimes cumbersome. Bass-Serre theory gives a nice tool to deal with subgroups of fundamental groups of graphs of groups, but the algorithmic side of the theory is still underdeveloped. It seems, the first general attempt to provide an algorithmic version of Bass-Serre subgroup theorem via foldings was made [5]. We refer to this paper for a brief survey on the membership problem in fundamental groups of graphs of groups.

In the present paper, we develop folded graph methods for finitely generated subgroups $H$ of finitely generated fully residually free groups $G$. Notice that such groups $G$ are subgroups of groups obtained from a free group by a finite sequence of extensions of centralizers, so the methods of Bass-Serre theory, in particular, the results from [5], can be in use. However, we elect to generalize directly the original Stallings method for free groups on fully residually free ones. Namely, using Kharlampovich-Myasnikov embedding theorem [6] we embed the group $G$ into the Lyndon's free $\mathbb{Z}[t]$-group $F^{\mathbb{Z}[t]}$. Notice that such embedding can be found effectively. This allows one to view the groups $G$ as subgroups of $F^{\mathbb{Z}[t]}$ given by a finite set of generators. Now, for a finitely generated subgroup $H$ of $F^{\mathbb{Z}[t]}$ we effectively construct a folded labeled graph $\Gamma$ which accepts precisely those elements of $F^{\mathbb{Z}[t]}$ (written in a normal form) which belong to $H$. To construct $\Gamma$ we use the representation of elements from $F^{\mathbb{Z}[t]}$ by infinite words in the alphabet $X$ (here $X$ is a basis of $F$ ) that was introduced in [10]. Thus, we may assume that the subgroup $H$ is generated by a finite set of infinite words. In this event, to build $\Gamma$ we mimic the 
classical Stallings folding algorithm replacing finite words by the infinite ones. This is not immediate, however, and it requires some new ideas. When $\Gamma$ is constructed we solve the membership problem for $H$ precisely in the same way as in free groups. In the subsequent paper we use these graphs $\Gamma$ associated with the subgroups $H$ of fully residually free groups to solve other algorithmic problems for $H$.

\section{2. $F^{\mathbb{Z}[t]}$ and Its Elements Viewed as Infinite Words}

In this section, at first, we review the embedding of $F^{\mathbb{Z}[t]}$ into the set of reduced infinite words. Then we describe normal forms for elements of $F^{\mathbb{Z}[t]}$ which arise from the embedding.

\subsection{Lyndon's free $\mathbb{Z}[t]$-group and infinite words}

Let $F=F(X)$ be a free non-abelian group with basis $X=\left\{x_{i} \mid i \in I\right\}$ and $\mathbb{Z}[t]$ be a ring of polynomials with integer coefficients in $t$. Let $X^{-1}=\left\{x_{i}^{-1} \mid i \in I\right\}$ and $X^{ \pm}=X \cup X^{-1}$. In [7], Lyndon introduced a $\mathbb{Z}[t]$-completion $F^{\mathbb{Z}[t]}$ of $F$, which it is called now Lyndon's free $\mathbb{Z}[t]$-group. It turns out that $F^{\mathbb{Z}[t]}$ can be described as a union of a sequence of extensions of centralizers (see [9])

$$
F=G_{0}<G_{1}<\cdots<G_{n}<\cdots,
$$

where $G_{i+1}$ is obtained from $G_{i}$ by extension of all cyclic centralizers in $G_{i}$ by a free abelian group of countable rank.

In was shown in [10] that elements of $F^{\mathbb{Z}[t]}$ can be viewed as infinite words defined in the following way. Let $A$ be a discretely ordered abelian group. By $1_{A}$ we denote the minimal positive element of $A$. Recall that if $a, b \in A$ then the closed segment $[a, b]$ is defined as $[a, b]=\{x \in A \mid a \leq x \leq b\}$. An $A$-word is a function of the type

$$
w:\left[1_{A}, \alpha_{w}\right] \rightarrow X^{ \pm},
$$

where $\alpha_{w} \in A, \alpha_{w} \geq 0$. The element $\alpha_{w}$ is called the length $|w|$ of $w$. By $\varepsilon$ we denote the empty word. We say that $w$ is reduced if $w(\alpha) \neq w(\alpha+1)^{-1}$ for any $1_{A} \leq \alpha<\alpha_{w}$. Then, as in a free group, one can introduce a partial multiplication $*$, an inversion, a word reduction etc. on the set of all $A$-words (infinite words) $W(A, X)$. We write $u \circ v$ instead of $u v$ if $|u v|=|u|+|v|$. All these definitions make it possible to develop infinite words techniques, which provide a very convenient combinatorial tool (for all the details we refer to [10]).

It was proved in [10] that $F^{\mathbb{Z}[t]}$ can be canonically embedded into the set of reduced infinite words $R(\mathbb{Z}[t], X)$, where $\mathbb{Z}[t]$, an additive group of polynomials with integer coefficients, is viewed as an ordered abelian group with respect to the standard lexicographic order $\leq$ (that is, the order which compares the degrees of polynomials first, and if the degrees are equal, compares the coefficients of corresponding terms starting with the terms of highest degree). More precisely, the embedding of $F^{\mathbb{Z}[t]}$ into $R(\mathbb{Z}[t], X)$ was constructed by induction, that is, all $G_{i}$ 
from the series (2.1) were embedded step by step in the following way. Suppose, the embedding of $G_{i}$ into $R(\mathbb{Z}[t], X)$ is already constructed. Then, one chooses a Lyndon's set $U_{i} \subset G_{i}$ (see [10]) and the extension of centralizers of all elements from $U_{i}$ produces $G_{i+1}$, which is now also naturally embedded into $R(\mathbb{Z}[t], X)$.

The existence of an embedding of $F^{\mathbb{Z}[t]}$ into the set of infinite words implies automatically the fact that all subgroups of $F^{\mathbb{Z}[t]}$ are also subsets of $R(\mathbb{Z}[t], X)$, that is, their elements can be viewed as infinite words. From now on we assume the embedding $\rho: F^{\mathbb{Z}[t]} \rightarrow R(\mathbb{Z}[t], X)$ to be fixed. Moreover, for simplicity we identify $F^{\mathbb{Z}[t]}$ with its image $\rho\left(F^{\mathbb{Z}[t]}\right)$.

Further we introduce an order on each $U_{i}$. Let $v \in U_{i}$. There exists an infinite subset $K_{i}(v) \subset U_{i}$ such that $u \in K_{i}(v)$ only if $|u|=|v|$. In fact, $K_{i}(u)=K_{i}(v)$ if and only if $u, v \in U_{i},|u|=|v|$. Thus, there exist elements $u_{1}^{i}, \ldots, u_{n}^{i}, \ldots$ in $U_{i}$ such that

$$
U_{i}=\bigcup_{j} K_{i}\left(u_{j}^{i}\right)
$$

By Zorn's Lemma we can assume $K_{i}\left(u_{j}^{i}\right), j \in \mathbb{N}$ to be well-ordered under some order $>_{j}$. If $u, v \in U_{i}$, then put

$$
u>v \text { iff }\left\{\begin{array}{l}
|u|>|v|, \\
\text { or, } \\
|u|=|v| \text { but } u>_{j} v, \text { where } u, v \in K_{i}\left(u_{j}^{i}\right) \text { for some } u_{j}^{i} .
\end{array}\right.
$$

Observe that $U_{i}$ is well-ordered under $>$ and enumerating its elements with respect to this order we get the set of indices $I_{i}$ of elements from $U_{i}$. The order on $U_{i}$ indices an order on $U=\bigcup_{i} U_{i}$ - if $u, v \in U_{i}$ then we compare them using the order defined on $U_{i}$ and if $u \in U_{i}, v \in U_{j}$ then $u>v$ if and only if $i>j$.

Now we introduce the notion of abelian height for elements from $\mathbb{Z}[t]$. Recall that $\mathbb{Z}[t]$ is a countable direct sum

$$
\mathbb{Z}[t]=\bigoplus_{i=0}^{\infty}\left\langle t^{i}\right\rangle
$$

of copies of the infinite cyclic group $\mathbb{Z}$ with the right lexicographic order. So, for every $\alpha \in \mathbb{Z}[t]$ there exists a natural number $n$ such that $\alpha$ belongs to

$$
\mathbb{Z}^{n}=\bigoplus_{i=0}^{n}\left\langle t^{i}\right\rangle
$$

Thus, we say that an abelian height $h_{a b}(\alpha)=n$ if $\alpha \in \mathbb{Z}^{n}-\mathbb{Z}^{n-1}$.

Compare the definition of abelian height with the definition of height given in [10]. Observe that if $w=u^{\alpha} \in R(\mathbb{Z}[t], X)$ then $h(w)=h(u) h_{a b}(\alpha)$.

\subsection{Reduced forms for elements of $\boldsymbol{F}^{\mathbb{Z}[t]}$}

We start with the following convention, which somehow simplifies notations used throughout the text. 
Conventions 2.1. From now on we call $\alpha \in \mathbb{Z}[t]$ non-standard if it does not belong to the "standard" part of $\mathbb{Z}[t]$ which is the smallest convex subgroup of $\mathbb{Z}[t]$. If $\alpha$ is non-standard then we write $\alpha \gg 0$.

An element $g \in G_{n+1}-G_{n}$ has the following representation as a reduced infinite word

$$
g=g_{1} \circ u_{n_{1}}^{\alpha_{1}} \circ g_{2} \circ \cdots \circ u_{n_{l}}^{\alpha_{l}} \circ g_{l+1},
$$

where $n_{1}, n_{2}, \ldots, n_{l} \in I_{n}, g_{k} \in G_{n}, k \in[1, l+1],\left[g_{k}, u_{n_{k}}\right] \neq \varepsilon,\left[g_{k+1}, u_{n_{k}}\right] \neq \varepsilon$, $k \in[1, l],\left|\alpha_{k}\right| \gg 0, k \in[1, l]$. Observe that this representation is not unique because for each $k \in[1, l]$ it is possible that $g_{k}$ has $u_{n_{k}}^{p_{k}}$ as a terminal segment and $g_{k+1}$ has $u_{n_{k}}^{m_{k}}$ as an initial segment, so we can adjoin these finite exponents of $u_{n_{k}}$ from the left and from the right to $u_{n_{k}}^{\alpha_{k}}$, to form a new infinite word representation of the same element $g$

$$
g=h_{1} \circ u_{n_{1}}^{\beta_{1}} \circ h_{2} \circ \cdots \circ u_{n_{l}}^{\beta_{l}} \circ h_{l+1},
$$

where $\beta_{k}=\alpha_{k}+p_{k}+m_{k}, g_{1}=h_{1} \circ u_{n_{1}}^{p_{1}}, g_{l+1}=u_{n_{l}}^{m_{l}} \circ h_{l+1}, g_{k}=u_{n_{k-1}}^{m_{k-1}} \circ h_{k} \circ u_{n_{k}}^{p_{k}}$, $k \in[2, l]$.

Remark 2.2. It is not hard to see that there are infinitely many representations of $g$ in the form (2.2) - it is possible not only to perform the transformation described above, that is, to adjoin finite exponents of $u_{n_{k}}, k \in[1, l]$ from the left and from the right to $u_{n_{k}}^{\alpha_{k}}$, but also to perform inverse transformations, that is, to take finite exponents from each $u_{n_{k}}^{\alpha_{k}}, k \in[1, l]$ in order to obtain infinitely many tuples of interleaving elements $f_{1}, \ldots, f_{l+1}$.

Lemma 2.3. Let $g \in G_{n+1}-G_{n}$ have two representations

$$
g=g_{1} \circ u_{s_{1}}^{\alpha_{1}} \circ g_{2} \circ \cdots \circ u_{s_{l}}^{\alpha_{l}} \circ g_{l+1},
$$

where $s_{j} \in I_{n}, g_{j} \in G_{n},\left[g_{j}, u_{s_{j}}\right] \neq \varepsilon,\left[g_{j+1}, u_{s_{j}}\right] \neq \varepsilon,\left|\alpha_{j}\right| \gg 0, j \in[1, l]$ and

$$
g=h_{1} \circ u_{r_{1}}^{\beta_{1}} \circ h_{2} \circ \cdots \circ u_{r_{l}}^{\beta_{l}} \circ h_{m+1},
$$

where $t_{k} \in I_{n}, h_{k} \in G_{n},\left[h_{k}, u_{r_{k}}\right] \neq \varepsilon,\left[h_{k+1}, u_{r_{k}}\right] \neq \varepsilon,\left|\beta_{k}\right| \gg 0, k \in[1, m]$. Then

(1) $l=m$,

(2) $u_{r_{j}}=u_{s_{j}}, j \in[1, l]$.

Proof. We have

$$
\left(g_{l+1}^{-1} \circ u_{s_{l}}^{-\alpha_{l}} \circ \cdots \circ u_{s_{1}}^{-\alpha_{1}} \circ g_{1}^{-1}\right) *\left(h_{1} \circ u_{r_{1}}^{\beta_{1}} \circ h_{2} \circ \cdots \circ u_{r_{l}}^{\beta_{l}} \circ h_{m+1}\right)=\varepsilon .
$$

By $\left[10\right.$, Lemma 6.9] the equality above can hold only if $u_{r_{1}}=u_{s_{1}}$ and $g_{1}^{-1} * h_{1}=u_{s_{1}}^{k_{1}}$. Thus, we have

$$
\left(g_{l+1}^{-1} \circ u_{s_{l}}^{-\alpha_{l}} \circ \cdots \circ g_{2}^{-1}\right) * u_{s_{1}}^{p_{1}} *\left(h_{2} \circ \cdots \circ u_{r_{l}}^{\beta_{l}} \circ h_{m+1}\right)=\varepsilon,
$$


where $p_{1}$ is finite. Again, by [10, Lemma 6.9], $u_{r_{2}}=u_{s_{2}}$ and $g_{2}^{-1} * u_{s_{1}}^{p_{1}} * h_{2}=u_{s_{2}}^{k_{2}}$ and we can cancel $\left(u_{s_{2}}^{-\alpha_{2}} \circ g_{2}^{-1}\right) * u_{s_{1}}^{p_{1}} *\left(h_{2} \circ u_{r_{2}}^{\beta_{2}}\right)$ into a finite exponent $u_{s_{2}}^{p_{2}}$. The required result now follows by induction.

It follows from Lemma 2.3 that in any representation of $g \in G_{n+1}-G_{n}$ as an infinite word, the number of non-standard exponents $u_{n_{k}}^{\alpha_{k}}$ in $g$ (the number of syllables in some sense) is the same, which is equal to $l$. This observation makes it possible to introduce a natural characterization of any representation of $g$ as an $l$-tuple $\left\{\left|\gamma_{1}\right|,\left|\gamma_{2}\right|, \ldots,\left|\gamma_{l}\right|\right\}$, where $\left|\gamma_{k}\right| \gg 0$ and

$$
g=f_{1} \circ u_{n_{1}}^{\gamma_{1}} \circ f_{2} \circ \cdots \circ u_{n_{l}}^{\gamma_{l}} \circ f_{l+1} .
$$

We call such a representation of $g, U_{n}$-reduced if the ordered $l$-tuple $\left\{\left|\gamma_{1}\right|\right.$, $\left.\left|\gamma_{2}\right|, \ldots,\left|\gamma_{l}\right|\right\}$ is maximal with respect to the right lexicographic order among all possible representations of $g$.

In [10] we introduced unique reduced forms for elements of $F^{\mathbb{Z}[t]}$. Now, we redefine the procedure for obtaining them and show that in fact unique reduced forms defined in the proof of [10, Theorem 6.15] coincide with $U_{n}$-reduced representations (in the future we will use the term $U_{n}$-form instead of $U_{n}$-reduced representation).

Suppose $g \in G_{n+1}-G_{n}$ and

$$
g=g_{1} \circ u_{n_{1}}^{\alpha_{1}} \circ g_{2} \circ \cdots \circ u_{n_{l}}^{\alpha_{l}} \circ g_{l+1} .
$$

From [10, Lemma 6.9] it follows that for $g_{1}$ and $g_{2}$ there exist $p_{1}, m_{1} \in \mathbb{N}$ such that $g_{1}=h_{1} \circ u_{n_{1}}^{p_{1}}, g_{2} \circ u_{n_{2}}^{\alpha_{2}}=u_{n_{1}}^{m_{1}} \circ g_{2}^{\prime} \circ u_{n_{2}}^{\gamma_{2}}$ and $h_{1}$ does not have $u_{n_{1}}^{ \pm 1}$ as a terminal segment, $g_{2}^{\prime} \circ u_{n_{2}}^{\gamma_{2}}$ does not have $u_{n_{1}}^{ \pm 1}$ as an initial segment. Now we present $g$ as

$$
g=h_{1} \circ u_{n_{1}}^{\beta_{1}} \circ g_{2}^{\prime} \circ u_{n_{2}}^{\gamma_{2}} \circ \cdots \circ u_{n_{l}}^{\alpha_{l}} \circ g_{l+1},
$$

where $\beta_{k}=\alpha_{k}+p_{k}+m_{k}, g_{1}=h_{1} \circ u_{n_{1}}^{p_{1}}, g_{2} \circ u_{n_{2}}^{\alpha_{2}}=u_{n_{1}}^{m_{1}} \circ g_{2}^{\prime} \circ u_{n_{2}}^{\gamma_{2}}$. Next we take the subword of $g$

$$
g^{\prime}=g_{2}^{\prime} \circ u_{n_{2}}^{\gamma_{2}} \circ g_{3} \circ \cdots \circ u_{n_{l}}^{\alpha_{l}} \circ g_{l+1}
$$

and perform exactly the same procedure of maximizing $\gamma_{2}$. The whole construction follows by induction on $l$. After a finite number of steps we get

$$
g=h_{1} \circ u_{n_{1}}^{\beta_{1}} h_{2} \circ \cdots \circ u_{n_{l}}^{\beta_{l}} \circ h_{l+1} .
$$

\section{Lemma 2.4.}

(1) (2.4) is unique,

(2) (2.4) is a $U_{n}$-reduced form for $g$.

Proof. (1) follows immediately from the construction.

(2) Suppose (2.4) is not a $U_{i}$-reduced form for $g$, that is, there exists an $l$-tuple $\left\{\left|\gamma_{1}\right|,\left|\gamma_{2}\right|, \ldots,\left|\gamma_{l}\right|\right\}$ such that $\left\{\left|\gamma_{1}\right|,\left|\gamma_{2}\right|, \ldots,\left|\gamma_{l}\right|\right\}>\left\{\left|\beta_{1}\right|,\left|\beta_{2}\right|, \ldots,\left|\beta_{l}\right|\right\}$ and

$$
g=f_{1} \circ u_{n_{1}}^{\gamma_{1}} \circ f_{2} \circ \cdots \circ u_{n_{l}}^{\gamma_{l}} \circ f_{l+1} .
$$


From the inequality above it follows that there exists $k_{0} \in[1, l]$ such that $\gamma_{k}=\beta_{k}$, $k \in\left[1, k_{0}-1\right]$ and $\gamma_{k_{0}}>\beta_{k_{0}}$.

At first we show that $h_{k}=f_{k}, k \in\left[1, k_{0}-1\right]$. Assume that $k_{0}>1$.

Observe that $\left|h_{1}\right| \leq\left|f_{1}\right|$ otherwise $h_{1}=f_{1} \circ u_{n_{1}}^{m},|m|>0, m \in \mathbb{Z}$ and $\beta_{1}$ is not maximal - contradiction (here we use [10, Lemma 6.9] from which it follows that $\left.f_{1}^{-1} * h_{1} \in\left\langle u_{n_{1}}\right\rangle\right)$. If $\left|h_{1}\right|<\left|f_{1}\right|$ then since $\beta_{1}=\gamma_{1}$ it follows that $h_{2} \circ u_{n_{2}}^{\beta_{2}}$ contains $u_{n_{1}}$ as an initial segment - contradiction with the choice of $\beta_{1}$. Thus $\left|h_{1}\right|=\left|f_{1}\right|$ and $h_{1}=f_{1}$. In the same way, using induction one can prove that $h_{k}=f_{k}, k \in\left[2, k_{0}-1\right]$.

The simple fact proved above shows that we can assume without loss of generality that $k_{0}=1$, that is, $\gamma_{1}>\beta_{1}>0$. Again, $\left|h_{1}\right| \leq\left|f_{1}\right|$ since otherwise we get a contradiction. As above if $\left|h_{1}\right|<\left|f_{1}\right|$ then since $\beta_{1}<\gamma_{1}$ it follows that $h_{2} \circ u_{n_{2}}^{\beta_{2}}$ contains at least $u_{n_{1}}$ as an initial segment - contradiction with the choice of $\beta_{1}$.

Thus we proved that $\left\{\left|\beta_{1}\right|,\left|\beta_{2}\right|, \ldots,\left|\beta_{l}\right|\right\}$ is the maximal $l$-tuple with respect to the right lexicographical order. So the representation (2.4) is $U_{n}$-reduced for $g$.

Let $g \in G_{n+1}-G_{n}$, so it has a representation as an infinite word

$$
g=g_{1} \circ u_{n_{1}}^{\alpha_{1}} \circ g_{2} \circ \cdots \circ u_{n_{l}}^{\alpha_{l}} \circ g_{l+1},
$$

where $n_{1}, n_{2}, \ldots, n_{l} \in I_{n}, g_{k} \in G_{n},\left[g_{k}, u_{n_{k}}\right] \neq \varepsilon,\left[g_{k+1}, u_{n_{k}}\right] \neq \varepsilon,\left|\alpha_{k}\right| \gg 0$. Now we fix some $u$ from the list $u_{n_{1}}, u_{n_{2}}, \ldots, u_{n_{l}}$ of elements from $U_{n}$ taken to non-standard powers in the representation of $g$ (it follows from Lemma 2.3 that this list does not depend on particular representation of $g$ as a reduced infinite word). Consider now a representation of $g$ in which we "mark" only non-standard exponents of $u$, that is,

$$
g=h_{1} \circ u^{\beta_{1}} \circ h_{2} \circ \cdots \circ u^{\beta_{p}} \circ h_{p+1},
$$

where $\beta_{j}=\alpha_{m_{j}}, m_{j} \in[1, l], j \in[1, p], h_{1}=g_{1} \circ u_{n_{1}}^{\alpha_{1}} \cdots \cdots g_{m_{1}}, h_{p+1}=g_{m_{p}+1} \circ \cdots \circ$ $g_{l+1}, h_{k}=g_{m_{k}+1} \circ \cdots \circ g_{m_{k+1}}, k \in[2, p]$. Observe that all $h_{k}$, in general, do not belong to $G_{n}$ any more. We call (2.5) the the u-representation or the $u$-form for $g$.

One can prove a statement analogous to Lemma 2.3 for $u$-forms of $g$ and this means that we can associate with any such form a $p$-tuple $\left\{\left|\beta_{1}\right|,\left|\beta_{2}\right|, \ldots,\left|\beta_{p}\right|\right\}$ of elements from $\mathbb{Z}[t]$, which are infinite exponents from $(2.5)$.

We call a $u$-form of $g, u$-reduced if the ordered $p$-tuple $\left\{\left|\beta_{1}\right|,\left|\beta_{2}\right|, \ldots,\left|\beta_{p}\right|\right\}$ is maximal with respect to the right lexicographic order among all possible $u$-forms for $g$.

Suppose

$$
h_{1} \circ u^{\beta_{1}} \circ h_{2} \circ \cdots \circ u^{\beta_{p}} \circ h_{p+1}
$$

is a $u$-form for $g$ and $g$ is cyclically reduced. Then, obviously

$$
\left(h_{1} \circ u^{\beta_{1}} \circ h_{2} \circ \cdots \circ u^{\beta_{p}} \circ h_{p+1}\right) \circ\left(h_{1} \circ u^{\beta_{1}} \circ h_{2} \circ \cdots \circ u^{\beta_{p}} \circ h_{p+1}\right)
$$

is a $u$-form of $g^{2}$. So, we call (2.6) cyclically u-reduced if (2.7) is $u$-reduced. 
Lemma 2.5. Let

$$
h_{1} \circ u^{\beta_{1}} \circ h_{2} \circ \cdots \circ u^{\beta_{p}} \circ h_{p+1}
$$

be a u-reduced form of $g \in G_{n+1}-G_{n}, u \in U_{i}$. Then there exists a cyclic permutation of $g$ such that its $u$-reduced form is cyclically $u$-reduced.

Proof. The proof is based on the following observation.

Claim 2.6. Let $|w|<|u|$. If $w \circ u^{\beta}$ has $u$ as an initial segment in $u^{\alpha} \circ w \circ u^{\beta}$, $\alpha, \beta \in \mathbb{Z}, \alpha, \beta>0$ then $u^{\alpha} \circ w$ cannot have $u$ as a terminal segment.

Suppose on the contrary that $u=w \circ u_{1}, u=u_{1} \circ u_{2}$ and $u=u_{3} \circ w, u=u_{4} \circ u_{3}$ at the same time. From these equalities we have $|w|=\left|u_{2}\right|,\left|u_{1}\right|=\left|u_{3}\right|,\left|u_{4}\right|=|w|$. Since $u=w \circ u_{1}, u=u_{4} \circ u_{3}$ and $\left|u_{4}\right|=|w|$ it follows that $w=u_{4}, u_{1}=u_{3}$. Thus $w=u_{2}$ and $u=u_{1} \circ u_{2}=u_{2} \circ u_{1}$, but this is possible only if $u_{1}=a^{\delta}$ and $u_{2}=a^{\gamma}$, and hence $u=a^{\delta+\gamma}$, so the centralizer of $u$ in $G_{n}$ is not cyclic - contradiction with the choice of $U_{n}$.

Observe also that if $\alpha>0, \beta<0$ then neither $w \circ u^{\beta}$ has $u$ as an initial segment nor $u^{\alpha} \circ w$ has $u^{-1}$ as a terminal segment. Indeed, if for example $w \circ u^{\beta}$ has $u$ as an initial segment then $u=w \circ u_{1}, u^{-1}=u_{1} \circ u_{2}$. Thus, $u=w \circ u_{1}=u_{2}^{-1} \circ u_{1}^{-1}$ and $u_{1}=u_{1}^{-1}=\varepsilon, u=w-$ contradiction.

Now we complete the proof of the lemma. Without loss of generality we can assume $h_{1}=\varepsilon$ and that $h_{p+1}$ does not have $u$ as a terminal segment (using cyclic permutation we can always obtain these properties). We have two cases

(1) $\left|h_{p+1}\right| \geq|u|$

Since $u^{\beta_{1}} \circ h_{2} \circ \cdots \circ u^{\beta_{p}} \circ h_{p+1}$ is $u$-reduced it follows that $u^{\beta_{1}} \circ h_{2} \circ \cdots \circ u^{\beta_{p}} \circ$ $h_{p+1} \circ u^{\beta_{1}} \circ h_{2} \circ \cdots \circ u^{\beta_{p}} \circ h_{p+1}$ is $u$-reduced because $h_{p+1}$ does not have $u$ as an initial or terminal segment.

(2) $\left|h_{p+1}\right|<|u|$

(a) If $u^{\beta_{p}} \circ h_{p+1}$ has $u$ as a terminal segment then by Claim $2.6, h_{p+1} \circ u^{\beta_{1}}$ does not have $u$ as an initial segment. Since $u^{\beta_{1}} \circ h_{2} \circ \cdots \circ u^{\beta_{p}} \circ h_{p+1}$ is $u$-reduced then the ordered $2 p$-tuple $\left\{\left|\beta_{1}\right|,\left|\beta_{2}\right|, \ldots,\left|\beta_{p}\right|,\left|\beta_{1}\right|,\left|\beta_{2}\right|, \ldots,\left|\beta_{p}\right|\right\}$ is maximal with respect to the right lexicographic order among all possible $u$-forms of $g^{2}$. So, $u^{\beta_{1}} \circ h_{2} \circ \cdots \circ$ $u^{\beta_{p}} \circ h_{p+1}$ is cyclically $u$-reduced.

(b) If $h_{p+1} \circ u^{\beta_{1}}$ has $u$ as an initial segment then by Claim 2.6, $u^{\beta_{p}} \circ h_{p+1}$ does not have $u$ as a terminal segment. Thus, take a cyclic permutation $g^{\prime}=$ $h_{2} \circ u^{\beta_{2}} \cdots \circ u^{\beta_{p}} \circ h_{p+1} \circ u^{\beta_{1}}$ of $g$ so that $h_{2} \circ u^{\beta_{2}} \cdots \circ u^{\beta_{p}+1} \circ b \circ u^{\beta_{1}-1}$ is a $u$-reduced form of $g^{\prime}$, where $u=h_{p+1} \circ a=a \circ b$. But then $\left(h_{2} \circ u^{\beta_{2}} \cdots \circ u^{\beta_{p}+1} \circ b \circ u^{\beta_{1}-1}\right) \circ$ $\left(h_{2} \circ u^{\beta_{2}} \cdots \circ u^{\beta_{p}+1} \circ b \circ u^{\beta_{1}-1}\right)$ is $u$-reduced.

\subsection{Standard decomposition of elements of $F^{\mathbb{Z}[t]}$}

In this section, from infinite word representation of elements from $F^{\mathbb{Z}[t]}$ we obtain their representation by finite words in some alphabet. 
Let $g \in F^{\mathbb{Z}[t]}$. Then $g \in G_{n+1}-G_{n}$ for some $n \in \mathbb{N}$ and $g$ has a $U_{n}$-reduced form

$$
g=g_{1} \circ u_{n_{1}}^{\alpha_{1}} \circ g_{2} \circ \cdots \circ u_{n_{l}}^{\alpha_{l}} \circ g_{l+1},
$$

where $u_{n_{1}}, u_{n_{2}}, \ldots, u_{n_{l}} \in U_{n}, g_{k} \in G_{n}, k \in[1, l+1],\left[g_{k}, u_{n_{k}}\right] \neq \varepsilon,\left[g_{k+1}, u_{n_{k}}\right] \neq \varepsilon$, $\left|\alpha_{k}\right| \gg 0, k \in[1, l]$. Now, by the induction on $n$ we can assume existence of a $U_{n-1}$-reduced form for each $g_{i}, i \in[1, l]$

$$
g_{i}=g(i)_{1} \circ u_{m_{1}}^{\beta_{m_{1}}} \circ g(i)_{2} \circ \cdots \circ u_{m_{s}}^{\beta_{m_{s}}} \circ g(i)_{s},
$$

where $u_{m_{1}}, \ldots, u_{m_{s}} \in U_{n-1},\left|\beta_{m_{k}}\right| \gg 0, k \in[1, s], g(i)_{k} \in G_{n-1}, k \in[1, s+1]$. One can get down to the free group $F$ with such a decomposition of $g$, where subwords between non-standard powers of elements from $U_{i}$ are represented step by step as $U_{i-1}$-forms, $i \in[1, n]$. Thus, from this decomposition one can form the following series for $g$ :

$$
\begin{aligned}
F & <H_{0,1}<H_{0,2}<\cdots<H_{0, k(0)}<H_{1,1}<\cdots<H_{1, k(1)} \\
& <\cdots<H_{n-1, k(n-1)}<H_{n, 1}<\cdots<H_{n, k(n)}
\end{aligned}
$$

where $H_{j, 1}, \ldots, H_{j, k(j)}$ are subgroups of $G_{j+1}$ which do not belong to $G_{j}$ and $H_{j, i}$ is obtained from $H_{j, i-1}$ by a centralizer extension of a single element $u_{j, i-1} \in$ $H_{j, i-1}<G_{j}$. Element $g$ belongs to $H_{n, k(n)}$ and does not belong to the previous terms. Series (2.8) is called the extension series for $g$.

Using the extension series above we can decompose $g$ in the following way: $g \in H_{n, k(n)}$ has a $u_{n, k(n)}$-reduced form

$$
g=h_{1} \circ u_{n, k(n)}^{\beta_{1}} \circ h_{2} \circ \cdots \circ u_{n, k(n)}^{\beta_{l}} \circ h_{l+1},
$$

where all $h_{j}, j \in[1, l+1]$ in their turn are $u_{n, k(n)-1}$-reduced forms representing elements from $H_{n, k(n)-1}$. This gives one a decomposition of $g$ related to its extension series. We call this decomposition the standard decomposition or standard representation of $g$.

Observe that for any $g \in F^{\mathbb{Z}[t]}$, its standard decomposition can be viewed as a finite word in the alphabet

$$
B=X^{ \pm} \cup\left\{u^{\alpha} \mid u \in U, \alpha \in \mathbb{Z}[t]-\mathbb{Z}\right\} .
$$

We denote this product by $\pi(g)$ so we have

$$
\pi(g)=\pi\left(h_{1}\right) u_{n, k(n)}^{\beta_{1}} \pi\left(h_{2}\right) \cdots u_{n, k(n)}^{\beta_{l}} \pi\left(h_{l+1}\right),
$$

where $\pi\left(h_{i}\right)$ is a finite product in the alphabet $B$ corresponding to $h_{i}$, and from now on, by the standard decomposition of an element $g$ we understand not the representation of $g$ as a reduced infinite word but the finite word $\pi(g)$.

Let $U(g)$ be a finite subset of $U$ such that $u \in U(g)$ only if $\pi(g)$ contains a letter $b_{i} \in B$ such that $b_{i}=u^{\alpha}, \alpha \in \mathbb{Z}[t]-\mathbb{Z}$. Observe that $U(g)$ is ordered with respect to the order induced from $U$, that is, we have

$$
U(g)=\left\{u_{1}, \ldots, u_{m}\right\}
$$


where $u_{i}<u_{j}$ if $i<j$ and $u_{m}=u_{n, k(n)}$. By $\max \{U(g)\}$ we denote the maximal element of $U(g)$.

If $u \in U(g)$ then by $\operatorname{deg}_{u}(g)$ we denote the maximal degree of infinite exponents of $u$ which appear in $\pi(g)$.

It is easy to see that in general $\pi\left(g_{1} \circ g_{2}\right) \neq \pi\left(g_{1}\right) \pi\left(g_{2}\right)$ and $\pi(g \circ g)=\pi(g) \pi(g)$ if and only if the $u$-reduced form of $g$ is cyclically $u$-reduced, where $u=\max \{U(g)\}$.

From the definition of Lyndon's set and the results of [10] it follows that if $R \subset G_{n}$ is a Lyndon's set then a set $R^{\prime}$ obtained from $R$ by cyclic decompositions of its elements is also a Lyndon's set. Thus, by Lemma 2.5 we can assume a $w$ reduced form of any $u \in U_{n}$ to be cyclically $w$-reduced, where $w=\max \{U(u)\}$. Hence, we can assume

$$
\pi(u \circ u)=\pi(u) \pi(u)
$$

for any $u \in U$.

\section{Graphs Labeled by Infinite $\mathbb{Z}[t]$-Words}

In this section we introduce the notion of $(\mathbb{Z}[t], X)$-graphs and describe their basic properties.

\subsection{Labeled graphs}

Using the notation introduced in the previous section we adjust basic notions from [4] to $F^{\mathbb{Z}[t]}$.

Definition 3.1. By a $(\mathbb{Z}[t], X)$-labeled directed graph $((\mathbb{Z}[t], X)$-graph $) \Gamma$ we mean the following.

(1) $\Gamma$ is a combinatorial graph where every edge has a direction and is labeled either by a letter from $X$ or by an infinite exponent $u^{\alpha} \in F^{\mathbb{Z}[t]}, u \in U, \alpha \in \mathbb{Z}[t], \alpha>0$, denoted $\mu(e)$;

(2) for each edge $e$ of $\Gamma$ we denote the origin of $e$ by $o(e)$ and the terminus of $e$ by $t(e)$.

For each edge $e$ of $(\mathbb{Z}[t], X)$-graph we introduce a formal inverse $e^{-1}$ of $e$ with label $\mu(e)^{-1}$ and the endpoints defined as $o\left(e^{-1}\right)=t(e), t\left(e^{-1}\right)=o(e)$, that is, the direction of $e^{-1}$ is reversed with respect to the direction of $e$. For the new edge $e^{-1}$ we set $\left(e^{-1}\right)^{-1}=e$. The new graph, endowed with this additional structure we denote by $\widehat{\Gamma}$. In fact, usually we will abuse notation by disregarding the difference between $\Gamma$ and $\widehat{\Gamma}$.

Now we have a partition $E(\widehat{\Gamma})=E(\Gamma) \cup \overline{E(\Gamma)}$ and we say that edges of $\Gamma$ are positively oriented in $\widehat{\Gamma}$, while their formal inverses $e^{-1}$ are negatively oriented in $\widehat{\Gamma}$.

Definition 3.2. A path $p$ in $\Gamma$ is a sequence of edges $p=e_{1} \cdots e_{k}$, where each $e_{i}$ is an edge of $\widehat{\Gamma}$ and the origin of each $e_{i}$ is the terminus of $e_{i-1}$. 
Observe that $\mu(p)=\mu\left(e_{1}\right) \cdots \mu\left(e_{k}\right)$ is a word in the alphabet $X^{ \pm} \cup\left\{u^{\alpha} \mid u \in U\right.$, $\alpha \in \mathbb{Z}[t]\}$ and we denote by $\overline{\mu(p)}$ the reduced infinite word $\mu\left(e_{1}\right) * \cdots * \mu\left(e_{k}\right)$.

We will be using two different notions of the length of a path $p=e_{1} \cdots e_{k}$ in $\Gamma$

(1) combinatorial length $|p|$ set equal to $k$, and

(2) word length $w l(p)=\sum_{i=1}^{k} l\left(\mu\left(e_{i}\right)\right)$.

In fact, from these two definitions above two possible meanings of an irreducible path arise: an irreducible path in combinatorial sense and an irreducible path in the sense that its label viewed as an infinite word in $F^{\mathbb{Z}[t]}$ is reduced. Here are formal definitions.

Definition 3.3. A path $p=e_{1} \cdots e_{k}$ in a $(\mathbb{Z}[t], X)$-graph $\Gamma$ is called reduced if $e_{i} \neq e_{i+1}^{-1}$ for all $i \in[1, k-1]$.

Definition 3.4. A path $p=e_{1} \cdots e_{k}$ in a $(\mathbb{Z}[t], X)$-graph $\Gamma$ is called label reduced if

(1) $p$ is reduced;

(2) $e_{k_{1}} \cdots e_{k_{2}}, k_{1} \leq k_{2}$ is a subpath of $p$ such that $\mu\left(e_{i}\right)=u^{\alpha_{i}}, u \in U, \alpha_{i} \in \mathbb{Z}[t]$, $i \in\left[k_{1}, k_{2}\right]$ and $\mu\left(e_{k_{1}-1}\right) \neq u^{\beta_{1}}, \mu\left(e_{k_{2}+1}\right) \neq u^{\beta_{2}}$ for any $\beta_{1}, \beta_{2} \in \mathbb{Z}[t]$, provided $k_{1}-1, k_{2}+1 \in[1, k]$, then $\alpha=\alpha_{k_{1}}+\cdots+\alpha_{k_{2}} \neq 0$ and $\mu\left(e_{k_{1}-1}\right) * u^{\alpha}=$ $\mu\left(e_{k_{1}-1}\right) \circ u^{\alpha}, u^{\alpha} * \mu\left(e_{k_{2}+1}\right)=u^{\alpha} \circ \mu\left(e_{k_{2}+1}\right)$.

\subsection{Free foldings}

Here we define free (partial) foldings and partially folded $(\mathbb{Z}[t], X)$-graphs. Observe that the definition of a partial folding below is exactly the same as the corresponding definition of a folding in free groups (see [4]).

Definition 3.5. Let $\Gamma$ be a finite $(\mathbb{Z}[t], X)$-graph and let $v$ be a vertex of $\Gamma$. The valence of $v$ denoted $\operatorname{val}(v)$ is the number of all edges in $\Gamma$ which have $v$ as an origin or a terminus.

Let $\Gamma$ be a $(\mathbb{Z}[t], X)$-graph. Suppose $v_{0}$ is a vertex of $\Gamma$ and $f_{1}, f_{2}$ are two distinct edges of $\widehat{\Gamma}$ such that $o\left(f_{1}\right)=o\left(f_{2}\right)=v_{0}, \mu\left(f_{1}\right)=\mu\left(f_{2}\right)=x \in X^{ \pm}$or $\mu\left(f_{1}\right)=\mu\left(f_{2}\right)=u^{\alpha}, u \in U, \alpha \in \mathbb{Z}[t]$. Let $h_{i}$ be the positive edge of $\Gamma$ corresponding to $f_{i}$ (that is, $h_{i}=f_{i}$ if $f_{i}$ is positive and $h_{i}=f_{i}^{-1}$ if $f_{i}$ is negative).

Let $\Delta$ be a $(\mathbb{Z}[t], X)$-graph with the following sets of vertices and edges.

$$
V(\Delta)=\left(V(\Gamma)-\left\{t\left(f_{1}\right), t\left(f_{2}\right)\right\}\right) \cup\{v\}, \quad E(\Delta)=\left(E(\Gamma)-\left\{h_{1}, h_{2}\right\}\right) \cup\{h\} .
$$

The endpoints and arrows for the edges of $\Delta$ are defined in the following way. Let $e \in E(\Delta), e \neq h$ then

(1) we put $o_{\Delta}(e)=o_{\Gamma}(e)$ if $o_{\Gamma}(e) \neq t\left(f_{i}\right)$ and $o_{\Delta}(e)=v$ if $o_{\Gamma}(e)=t\left(f_{i}\right)$ for some $i$,

(2) we put $t_{\Delta}(e)=t_{\Gamma}(e)$ if $t_{\Gamma}(e) \neq t\left(f_{i}\right)$ and $t_{\Delta}(e)=v$ if $t_{\Gamma}(e)=t\left(f_{i}\right)$ for some $i$. 
For the edge $h$ we put $o_{\Delta}(h)=v_{0}, t_{\Delta}(h)=v$ if $h_{1}=f_{1}, h_{2}=f_{2}$ and $o_{\Delta}(h)=v, t_{\Delta}(h)=v_{0}$ otherwise.

We define labels on the edges of $\Delta$ as follows: $\mu_{\Delta}(e)=\mu_{\Gamma}(e)$ if $e \neq h$ and $\mu_{\Delta}(h)=\mu_{\Gamma}\left(h_{1}\right)=\mu_{\Gamma}\left(h_{2}\right)$.

In other words we obtain $\Delta$ by identification of two edges $f_{1}$ and $f_{2}$ in $\Gamma$. In this situation we say that $\Delta$ is obtained from $\Gamma$ by a free (partial) folding (or by freely (partially) folding the edges $f_{1}$ and $f_{2}$ ).

There can be introduced a notion of a morphism between two $(\mathbb{Z}[t], X)$-graphs. That is, if $\Gamma_{1}, \Gamma_{2}$ are $(\mathbb{Z}[t], X)$-graphs then a map $\theta: \Gamma_{1} \rightarrow \Gamma_{2}$ is called a morphism of $(\mathbb{Z}[t], X)$-graphs, if $\theta$ sends vertices to vertices, directed edges to directed edges, preserves labels of directed edges, and has the property that $o(\theta(e))=$ $\theta(o(e)), t(\theta(e))=\theta(t(e))$ for any edge $e$ of $\Gamma_{1}$.

If $\phi$ is a partial folding defined above then it is easy to see that $\phi$ is a morphism between $\Gamma$ and $\Delta$.

Lemma 3.6. Let $\Gamma_{1}$ be a $(\mathbb{Z}[t], X)$-graph obtained by a free folding from a graph $\Gamma$. Let $v$ be a vertex of $\Gamma$ and $v_{1}$ be the corresponding vertex of $\Gamma_{1}$. Then the following hold.

(1) If $\Gamma$ is connected then $\Gamma_{1}$ is connected.

(2) Let $p$ be the path from $v$ to $v$ in $\Gamma$ with label $w$. Then the edgewise image of $p$ in $\Gamma_{1}$ is a path from $v_{1}$ to $v_{1}$ with label $w$.

(3) If $\Gamma$ is a finite $(\mathbb{Z}[t], X)$-graph, then the number of edges in $\Gamma_{1}$ is one less than the number of edges in $\Gamma$, that is, any free folding decreases the number of edges in $\Gamma$.

Proof. Follows directly from the definition of a free folding.

Definition 3.7. ( $\mathbb{Z}[t], X)$-graph $\Gamma$ is called partially folded if there exist no two edges $e_{1}$ and $e_{2}$ in $\Gamma$ with $\mu\left(e_{1}\right)=\mu\left(e_{2}\right)$ such that $o\left(e_{1}\right)=o\left(e_{2}\right)$ or $t\left(e_{1}\right)=t\left(e_{2}\right)$.

Obviously, $\Gamma$ is a partially folded $(\mathbb{Z}[t], X)$-graph if and only if one cannot perform any free folding in $\Gamma$. Moreover the following proposition is true.

Proposition 3.8. Let $\Gamma$ be a $(\mathbb{Z}[t], X)$-graph, which has only a finite number of edges. Then there exists a partially folded $(\mathbb{Z}[t], X)$-graph $\Delta$, which can be obtained from $\Gamma$ by a finite number of free foldings.

Proof. Since $\Gamma$ has a finite number of edges by Lemma 3.6 any $(\mathbb{Z}[t], X)$-graph $\Gamma_{1}$ obtained from $\Gamma$ by a free folding has fewer edges. This provides one with an inductive argument based on the number of edges in $\Gamma$. 


\section{3. u-components}

In the present subsection we concentrate on some particular subgraphs of $(\mathbb{Z}[t], X)$ graphs which consist of edges labeled by exponents of elements from $U$. These subgraphs are very important in all our further investigations.

Let $u \in U$ be fixed.

Definition 3.9. Let $\Gamma$ be a $(\mathbb{Z}[t], X)$-graph. Vertices $v_{1}, v_{2} \in V(\Gamma)$ are called $u$ equivalent $\left(v_{1} \sim_{u} v_{2}\right)$ if there exists a path $p=e_{1} \cdots e_{k}$ in $\Gamma$ such that $o\left(e_{1}\right)=$ $v_{1}, t\left(e_{k}\right)=v_{2}$ and $\mu\left(e_{i}\right)=u_{i}^{\alpha}, \alpha_{i} \in \mathbb{Z}[t], i \in[1, k]$.

$\sim_{u}$ is clearly an equivalence relation on vertices of $\Gamma$, so if $\Gamma$ is finite then all its vertices can be divided into a finite number of pairwise disjoint equivalence classes. Suppose $v \in V(\Gamma)$ is fixed. One can take the subgraph of $\Gamma$ spanned by vertices $u$-equivalent to $v$ and remove from it all edges labeled by anything except for $u^{\alpha}, \alpha \in \mathbb{Z}[t]$. The resulting subgraph of $\Gamma$ we denote by $C o m p u(v)$ and call the $u$-component of $v$. In other words, the $u$-component of a vertex $v$ is the subgraph of $\Gamma$ all edges of which are labeled by exponents of $u$.

Definition 3.10. Let $\Gamma$ be a $(\mathbb{Z}[t], X)$-graph and $v \in V(\Gamma), v_{0} \in V\left(C o m p_{u}(v)\right)$. We define a set $H_{u}\left(v_{0}\right)$ associated with $v_{0}$ as

$$
H_{u}\left(v_{0}\right)=\left\{\overline{\mu(p)} \mid p \text { is a reduced path in } \operatorname{Comp}_{u}(v) \text { from } v_{0} \text { to } v_{0}\right\} \text {. }
$$

Observe that even when $p$ is a reduced path in $\operatorname{Comp}_{u}(v)$ its label $\mu(p)$ may be the empty infinite word.

Lemma 3.11. Let $\Gamma$ be $a(\mathbb{Z}[t], X)$-graph and $v \in V(\Gamma), v_{0} \in V\left(C o m p_{u}(v)\right)$. Then

(1) $H_{u}\left(v_{0}\right)$ is isomorphic to a subgroup of $\mathbb{Z}[t]$ and moreover, if $\operatorname{Comp}_{u}(v)$ is a finite graph then $H_{u}\left(v_{0}\right)$ is finitely generated,

(2) if $v_{1} \in V\left(\operatorname{Comp}_{u}(v)\right)$ then $H_{u}\left(v_{0}\right) \simeq H_{u}\left(v_{1}\right)$.

Proof. (1) Observe that if $p$ is a cycle in $\operatorname{Comp}_{u}(v)$ at $v_{0}$ then $\overline{\mu(p)}=u^{\alpha}, \alpha \in \mathbb{Z}[t]$. The concatenation $p_{1} p_{2}$ of two cycles in $C o m p_{u}(v)$ at $v_{0}$ is again a cycle in $C o m p_{u}(v)$ at $v_{0}$ which may or may not be reduced. Let $p$ be the reduced cycle obtained from

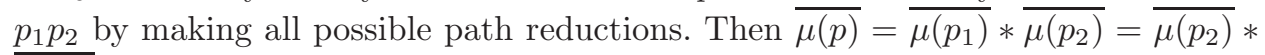
$\overline{\mu\left(p_{1}\right)} \in H_{u}\left(v_{0}\right)$ and $H_{u}\left(v_{0}\right)$ is closed under multiplication which is commutative.

Finally, since the inverse path $\left(p_{1}\right)^{-1}$ of $p_{1}$ is reduced and is labeled by $\mu\left(p_{1}\right)^{-1}$, it follows that $H_{u}\left(v_{0}\right)$ is closed under taking inverses. Also, since $\varepsilon$ is a label of an empty path which is reduced then clearly $\varepsilon \in H_{u}\left(v_{0}\right)$.

Thus, $H_{u}\left(v_{0}\right)$ is an abelian group.

One can construct a map $\theta: H_{u}\left(v_{0}\right) \rightarrow \mathbb{Z}[t]$, where $\overline{\mu(p)}=u^{\alpha} \stackrel{\theta}{\rightarrow} \alpha \in \mathbb{Z}[t]$. Obviously, $\theta$ is an isomorphism of $H_{u}\left(v_{0}\right)$ and a subgroup of $\mathbb{Z}[t]$. Moreover, if $\operatorname{Comp}_{u}(v)$ is a finite graph then there exists a natural number $n$ such that $n \geq$ $h_{a b}(\alpha)$, where $\mu(e)=u^{\alpha}$ and $e$ ranges through all edges of $\operatorname{Comp}_{u}(v)$. Since for any reduced cycle $p=e_{1} \cdots e_{k}$ in $\operatorname{Comp}_{u}(v)$ such that $\mu\left(e_{i}\right)=u^{\alpha_{i}}, i \in[1, k]$ and 
$\overline{\mu(p)}=u^{\alpha_{1}+\cdots+\alpha_{k}}=u^{\alpha}$, one has $h_{a b}(\alpha) \leq \max _{i=1}^{k}\left\{h_{a b}\left(\alpha_{i}\right)\right\}$ and it follows that $H_{u}\left(v_{0}\right)$ is a subgroup of $\mathbb{Z}^{n}$ which is a a finite rank subgroup of $\mathbb{Z}[t]$. Thus $H_{u}\left(v_{0}\right)$ is a free abelian group of rank not greater than $n$.

(2) Since $v_{0} \sim_{u} v_{1}$, there exists a path $p=f_{1} \cdots f_{k}$ in $\operatorname{Comp}_{u}(v)$ such that $o(p)=v_{0}, t(p)=v_{1}$ and $\overline{\mu(p)}=u^{\gamma}, \gamma \in \mathbb{Z}[t]$. The existence of $p$ provides one with correspondence between cycles at $v_{0}$ and $v_{1}$ - if $p_{0}=e_{1} \cdots e_{m}$ is a reduced cycle at $v_{1}$ then $p_{1}=f_{1} \cdots f_{k} e_{1} \cdots e_{m}\left(f_{k}\right)^{-1} \cdots\left(f_{1}\right)^{-1}$ is a cycle at $v_{0}$ and one can obtain a reduced cycle $p_{2}$ at $v_{0}$ by making all possible path reductions in $p_{1}$. Observe that $\left.\overline{\mu\left(p_{2}\right)}=\overline{\mu\left(p_{1}\right)}=\overline{\mu(p)} * \overline{\mu\left(p_{0}\right)} * \overline{(\mu(p)}\right)^{-1}=\overline{\mu\left(p_{0}\right)}$. Thus $H_{u}\left(v_{1}\right) \leq H_{u}\left(v_{0}\right)$. In the same way for any cycle at $v_{0}$ one can construct a corresponding cycle at $v_{1}$ with the same label. So $H_{u}\left(v_{1}\right) \geq H_{u}\left(v_{0}\right)$ and hence $H_{u}\left(v_{1}\right) \simeq H_{u}\left(v_{0}\right)$.

It follows from Lemma 3.11 that one can associate a free abelian group of finite rank with any finite $u$-component in a $(\mathbb{Z}[t], X)$-graph $\Gamma$.

\section{4. u-folded u-components}

In Sec. 3.2 we introduced a notion of free foldings, but it turns out that for our further investigations it is not enough to have only partially folded graphs. In the present subsection we introduce the notion of $U$-foldings which are operations on partially folded $(\mathbb{Z}[t], X)$-graphs.

Let $\Gamma$ be a $(\mathbb{Z}[t], X)$-graph and let $u \in U$ be fixed throughout this subsection. Let $v \in V(\Gamma), v_{0} \in V\left(\operatorname{Comp}_{u}(v)\right)$.

Free foldings defined in the previous subsection are simple operations on edges which have the same labels, but they do not cover the case when we have two edges $f_{1}, f_{2} \in E\left(\operatorname{Comp}_{u}(v)\right)$ such that $o\left(f_{1}\right)=o\left(f_{2}\right)=v_{0}, \mu\left(f_{1}\right)=u^{\alpha}, \mu\left(f_{2}\right)=u^{\beta}, u \in$ $U, \alpha, \beta \in \mathbb{Z}[t], \alpha \neq \beta$. Since $\alpha \neq \beta$, no free folding can be applied.

Definition 3.12 (u-folding). Let $f_{1}, f_{2} \in E\left(\operatorname{Comp}_{u}(v)\right)$ be such that $o\left(f_{1}\right)=$ $o\left(f_{2}\right)=v_{0}$ such that $\mu\left(f_{1}\right)=u^{\alpha}, \mu\left(f_{2}\right)=u^{\beta}, \alpha, \beta \in \mathbb{Z}[t]$ and suppose $|\alpha| \geq|\beta|$. Without loss of generality we can assume both edges to be positively oriented, that is, $\alpha, \beta>0$ (otherwise we can consider $f_{i}^{-1}, i=1,2$ instead of $f_{i}$ ).

Let $\Gamma_{1}$ be a $(\mathbb{Z}[t], X)$-graph defined as follows.

$$
V\left(\Gamma_{1}\right)=V(\Gamma) \cup\left\{v_{1}\right\}, \quad E\left(\Gamma_{1}\right)=\left(E(\Gamma)-\left\{f_{1}\right\}\right) \cup\left\{e_{1}, e_{2}\right\}
$$

We think of $\Gamma_{1}$ as a new $(\mathbb{Z}[t], X)$-graph obtained from $\Gamma$ by dividing the edge $f_{1}$ into two edges $e_{1}$ and $e_{2}$. The endpoints and arrows for the edges of $\Gamma_{1}$ are defined in the following way. Let $e \in E\left(\Gamma_{1}\right), e \neq e_{1}, e_{2}$ then we put $o_{\Gamma_{1}}(e)=o_{\Gamma}(e)$ and $t_{\Gamma_{1}}(e)=t_{\Gamma}(e)$

For the edges $e_{1}, e_{2}$ we put $o_{\Gamma_{1}}\left(e_{1}\right)=o_{\Gamma}\left(f_{1}\right), t_{\Gamma_{1}}\left(e_{1}\right)=v_{1}, o_{\Gamma_{1}}\left(e_{2}\right)=$ $v_{1}, t_{\Gamma_{1}}\left(e_{2}\right)=t_{\Gamma}\left(f_{1}\right)$.

Finally, $\mu_{\Gamma_{1}}(e)=\mu_{\Gamma}(e)$ if $e \neq e_{1}, e_{2}$ and $\mu_{\Gamma_{1}}\left(e_{1}\right)=\mu_{\Gamma}\left(f_{2}\right), \mu_{\Gamma_{1}}\left(e_{2}\right)=u^{\alpha-\beta}$. 
1.

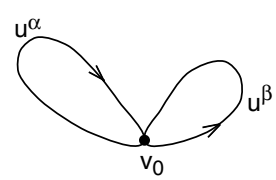

2.

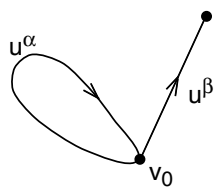

3.

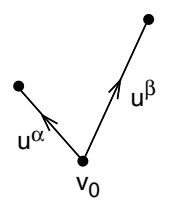

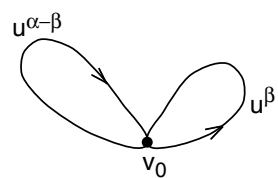
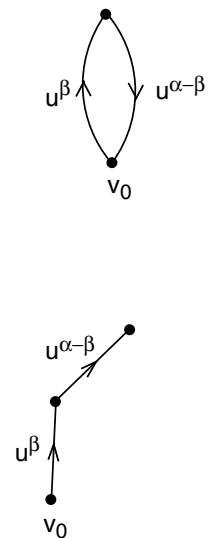

Fig. 1. Possible $u$-foldings.

Thus, in $\Gamma_{1}$ we have a pair of edges $e_{1}, f_{2}$ with origin $v_{0}$ and the same label $u^{\beta}$, so we can apply a free folding $\psi$ to $\Gamma_{1}$. After the identification of $e_{1}$ and $f_{2}$ the resulting $(\mathbb{Z}[t], X)$-graph we denote by $\Delta$.

In this situation we say that $\Delta$ is obtained from $\Gamma$ by a $u$-folding (or by $u$-folding the edges $f_{1}$ and $f_{2}$ ) (see Fig. 1 ).

Observe that if $\alpha=\beta$ then the $u$-folding defined above is just a free folding.

From now on by $U$-foldings we denote the set of all $u$-foldings, where $u \in U$.

Unlike free foldings, $u$-foldings do not define morphisms of $(\mathbb{Z}[t], X)$-graphs because they involve the operation of division of an edge. Hence, we introduce a notion of generalized morphism between two $(\mathbb{Z}[t], X)$-graphs. That is, if $\Gamma_{1}, \Gamma_{2}$ are $(\mathbb{Z}[t], X)$-graphs then a map $\theta: \Gamma_{1} \rightarrow \Gamma_{2}$ is called a generalized morphism of $(\mathbb{Z}[t], X)$-graphs, if $\theta$ sends vertices to vertices, directed edges to reduced paths and has the property that $o(\theta(p))=\theta(o(p)), t(\theta(p))=\theta(t(p)), \overline{\mu(\theta(p))}=\overline{\mu(p)}$ for any reduced path $p$ of $\Gamma_{1}$.

Let $\phi$ be a $u$-folding defined above which is applied to the pair of edges $\left\{f_{1}, f_{2}\right\}$ in $E\left(C o m p_{u}(v)\right)$. By definition, $\phi$ involves as a final stage a free folding $\psi$. Then we have $\phi\left(f_{1}\right)=\psi\left(e_{1}\right) \psi\left(e_{2}\right), \phi(e)=\psi(e), e \in E(\Gamma), e \neq f_{1}$ and $\phi(w)=$ $\psi(w), w \in V(\Gamma)$. Observe that it follows from the definition of $\phi$ that $o(\phi(e))=$ $o(\psi(e))=\psi(o(e))=\phi(o(e)), t(\phi(e))=t(\psi(e))=\psi(t(e))=\phi(t(e)), \mu(\phi(e))=\mu(e)$ for any $e \in E(\Gamma), e \neq f_{1}$ and $o\left(\phi\left(f_{1}\right)\right)=o\left(\psi\left(e_{1}\right)\right)=\psi\left(o\left(e_{1}\right)\right)=\phi\left(o\left(f_{1}\right)\right)$, $t\left(\phi\left(f_{1}\right)\right)=t\left(\psi\left(e_{2}\right)\right)=\psi\left(t\left(e_{2}\right)\right)=\phi\left(t\left(f_{1}\right)\right), \overline{\mu\left(\phi\left(f_{1}\right)\right)}=\mu\left(f_{1}\right)$. Hence, it follows 
that $o(\phi(p))=\phi(o(p)), t(\phi(p))=\phi(t(p)), \overline{\mu(\phi(p))}=\overline{\mu(p)}$ for any reduced path $p$ in $\Gamma$. Observe that $\phi(p)$ may not be reduced.

Thus, we verified that $u$-folding is a generalized morphism of $(\mathbb{Z}[t], X)$-graphs. The following result is analogous to Lemma 3.6 about free foldings.

Lemma 3.13. Let $\Gamma_{1}$ be a $(\mathbb{Z}[t], X)$-graph obtained by a u-folding from a $(\mathbb{Z}[t], X)$ graph $\Gamma$. Let $v$ be a vertex of $\Gamma$ and $v_{1}$ be the corresponding vertex of $\Gamma_{1}$. Then the following hold.

(1) If $\Gamma$ is connected then $\Gamma_{1}$ is connected.

(2) Let $p$ be the path from $v$ to $v$ in $\Gamma$ such that $\overline{\mu(p)}=w$. Then the image of $p$ in $\Gamma_{1}$ is a path $p_{1}$ from $v_{1}$ to $v_{1}$ such that $\overline{\mu\left(p_{1}\right)}=w$.

(3) If $\Gamma$ is a finite $(\mathbb{Z}[t], X)$-graph, then $\left|V\left(\Gamma_{1}\right)\right| \leq|V(\Gamma)|$.

Proof. (1) and (3) follow directly from the definition and (2) follows from the fact that $u$-foldings are generalized morphisms.

It is easy to see the difference between Lemma 3.6 and Lemma 3.13 above. Unlike free foldings, $u$-foldings do not preserve labels of paths just because of the division of edges involved, but any path in $\Gamma$ and its image in $\Gamma_{1}$ have the same labels viewed as reduced infinite words.

The following important result follows directly from Lemma 4.3 which will be proved in Sec. 4.2 in more general context.

Lemma 3.14. Let $\Delta$ be a $(\mathbb{Z}[t], X)$-graph obtained by a u-folding $\phi$ from a graph $\Gamma$. Let $v$ be a vertex of $\Gamma$ such that $v$ belongs to some $u$-component in $\Gamma$. Then $\phi(v)$ belongs to a u-component in $\Delta$ and $H_{u}(v) \simeq H_{u}(\phi(v))$.

Any finite $u$-component can be transformed into a single positively oriented path with an associated free abelian group of finite rank. The next results show how one can use $u$-foldings to get such a form of $u$-components.

At first, recall that in a connected graph a subgraph is said to be a spanning tree if this subgraph is a tree and it contains all vertices of the original graph. If a graph $T$ is a tree then for any two vertices $v_{1}, v_{2}$ of $T$ there is a unique reduced path in $T$ from $v_{1}$ to $v_{2}$.

Let $\Gamma$ be a $(\mathbb{Z}[t], X)$-graph and $v \in V(\Gamma)$. We call a path $p=e_{1} \cdots e_{k}$ in $C_{\text {Comp }}(v)$ positively oriented (negatively oriented) if $\alpha_{i}>0\left(\alpha_{i}<0\right), i \in[1, k]$, where $\mu\left(e_{i}\right)=u^{\alpha_{i}}$.

Lemma 3.15. Let $T$ be a finite tree such that all its edges are labeled by $u^{\alpha}$, $\alpha \in \mathbb{Z}[t]$ and let $v_{0} \in V(T)$. Then $T$ can be transformed by finitely many $u$-foldings into a tree $T^{\prime}$ such that if $v_{0}^{\prime} \in V\left(T^{\prime}\right)$ corresponds to $v_{0}$ then for any $v \in V\left(T^{\prime}\right)$ the unique reduced path $p_{v}$ from $v_{0}^{\prime}$ to $v$ is either positively oriented or negatively oriented. 
Proof. Since $v_{0}$ can be connected by a unique reduced path to any $v \in V(T)$ and $T$ is finite we have a finite number of such paths $p_{v}, v \in V(T)$. In each path $p_{v}=e_{1} \cdots e_{k}$ there can be positive edges, that is, labeled by $u^{\alpha}, \alpha>0$ and also negative edges. To prove the statement of the lemma we use induction on the number of vertices in $T$ such that $p_{v}$ is neither positively nor negatively oriented. Let $B_{T}$ denote the set of such vertices in $T$.

If $\left|B_{T}\right|=0$ then the lemma is already true for $T$. Thus we assume the statement to be true for any tree $S$ with $\left|B_{S}\right|=n-1$.
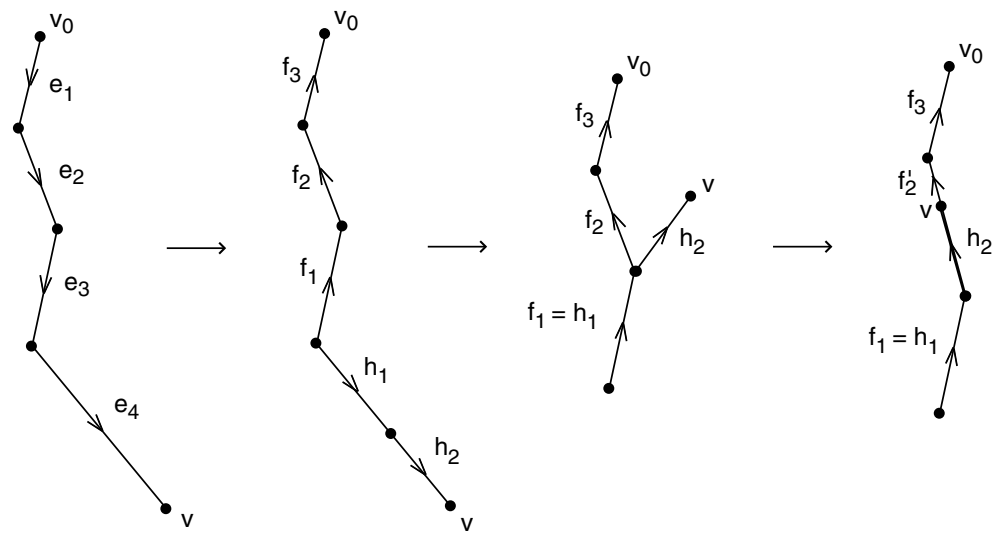

Fig. 2. Case (1), $j=4, i=2$.

Let $\left|B_{T}\right|=n$ and take any vertex $v$ from $B_{T}$. Then $p_{v}=e_{1} \cdots e_{k}$ is a unique reduced path such that $o(p)=v_{0}, t(p)=v, \mu\left(e_{i}\right)=u^{\alpha_{i}}, i \in[1, k]$. Without loss of generality we can assume $\alpha_{1}>0$. Since $v \in B$ there exists minimal $j \in[2, k]$ such that $\alpha_{j-1}>0, \alpha_{j}<0$. Thus $t\left(e_{j}\right) \in B_{T}$ and again, without loss of generality we can assume $v=t\left(e_{j}\right)$. We have two cases.

(1) $\alpha_{1}+\cdots+\alpha_{j-1} \geq\left|\alpha_{j}\right|$. There exists maximal $i \in[1, j-1]$ such that $\alpha_{i}+\cdots+\alpha_{j-1}>\left|\alpha_{j}\right|$ but $\alpha_{i+1}+\cdots+\alpha_{j-1}<\left|\alpha_{j}\right|$. Hence, $e_{j}$ can be folded step by step with the negatively oriented path $e_{j-1}^{-1} \cdots e_{i}^{-1}=f_{1} \cdots f_{j-i}, e_{j-s}^{-1}=f_{s}, s \in$ $[1, j-i]$ as follows.

$e_{j}$ is divided into negative edges $h_{1}, \ldots, h_{j-i}$ by new vertices $v_{1}, \ldots, v_{j-i-1}$ so that $o\left(h_{1}\right)=o\left(e_{j}\right), t\left(h_{s}\right)=o\left(h_{s+1}\right)=v_{s}, s \in[1, j-i-1], t\left(h_{j-i}\right)=t\left(e_{j}\right), \mu\left(h_{s}\right)=$ $\mu\left(f_{s}\right)=u^{-\alpha_{j-s}}, s \in[1, j-i-1], \mu\left(h_{j-i}\right)=u^{\alpha_{j}+\alpha_{i-1}+\cdots+\alpha_{j-1}}$. Finally, the sequence of $u$-foldings identifies each $h_{s}$ with $f_{s}$ for all $s \in[1, j-i-1]$ and $h_{j-i}$ becomes an initial part of $f_{j-i}=e_{i}^{-1}$. We denote this sequence of $u$-foldings by $\phi$ and let $T^{\prime}$ denote the result of applying $\phi$ to $T$. Observe that after $\phi$ is applied $v=t\left(e_{j}\right)$ is identified with a point on edge $e_{i}$, so $\phi(v)$ is connected to $v_{0}$ by a unique reduced positively oriented path. Obviously $T^{\prime}$ is also a tree and we have that $\phi(v) \notin B_{T^{\prime}}$. Observe also that if $w \notin B_{T}$ then $\phi(w) \notin B_{T^{\prime}}$. Indeed, if a vertex $w \in T$ is connected to $v_{0}$ by a unique path $p_{w}$ such that $e_{j} \in p_{w}$ then $p_{w}$ also contains $e_{1} \cdots e_{j-1}$ and 
$w \in B_{T}$. But such vertices are the only ones for which a path leading to $v_{0}$ is changed under $\phi$. So if $w^{\prime} \notin B_{T}$ then $p_{w^{\prime}}$ is not affected by $\phi$ and $\phi\left(p_{w^{\prime}}\right)=p_{w^{\prime}}$. It follows that $\left|B_{T^{\prime}}\right|<\left|B_{T}\right|$.
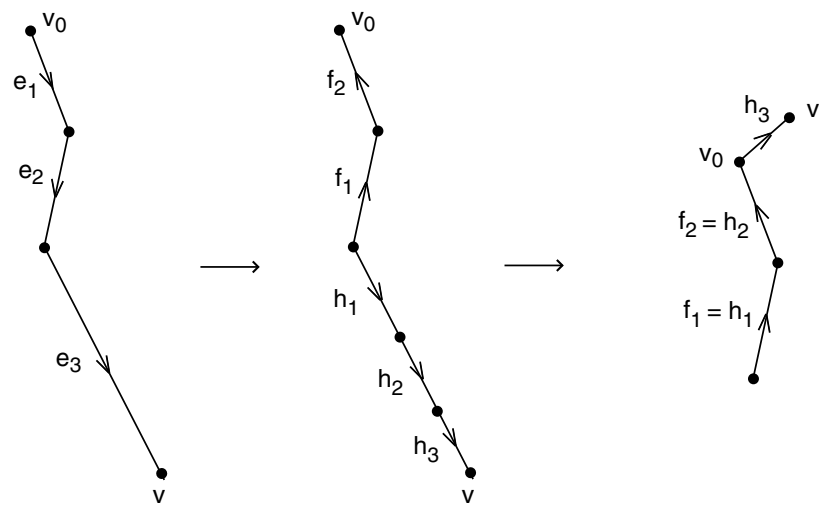

Fig. 3. Case (2), $j=3$.

(2) $\alpha_{1}+\cdots+\alpha_{j-1}<\left|\alpha_{j}\right| . \quad e_{j}$ can be folded step by step with the negatively oriented path $e_{j-1}^{-1} \cdots e_{1}^{-1}=f_{1} \cdots f_{j-1}, e_{j-s}^{-1}=f_{s}, s \in[1, j-1]$ as follows.

$e_{j}$ is divided into negative edges $h_{1}, \ldots, h_{j}$ by new vertices $v_{1}, \ldots, v_{j-1}$ so that $o\left(h_{1}\right)=o\left(e_{j}\right), t\left(h_{s}\right)=o\left(h_{s+1}\right)=v_{s}, s \in[1, j-1], t\left(h_{j}\right)=t\left(e_{j}\right), \mu\left(h_{s}\right)=\mu\left(f_{s}\right)=$ $u^{-\alpha_{j-s}}, s \in[1, j-1], \mu\left(h_{j-1}\right)=u^{\alpha_{j}+\alpha_{1}+\cdots+\alpha_{j-1}}$. Finally, the sequence of $u$-foldings identifies each $h_{s}$ with $f_{s}$ for all $s \in[1, j-1]$. We denote this sequence of $u$-foldings by $\phi$ and let $T^{\prime}$ denote the result of applying $\phi$ to $T$. Then $T^{\prime}$ is also a tree and we have that $\phi(v)=\phi\left(t\left(e_{j}\right)\right)$ is connected to $\phi\left(v_{0}\right)=v_{0}$ in $T^{\prime}$ by a unique reduced path which consists of a single negative edge $h_{j}$. So $\phi(v) \notin B_{T^{\prime}}$ and by the same argument as in (1) we have that $\left|B_{T^{\prime}}\right|<\left|B_{T}\right|$.

Thus, in both cases we obtained a new tree $T^{\prime}$ for which the statement can be obtained by induction.

As a corollary of Lemma 3.15 we get the following important result.

Corollary 3.16. Let $T$ be a finite tree such that all its edges are labeled by $u^{\alpha}$, $\alpha \in \mathbb{Z}[t]$ and let $v_{0} \in V(T)$. Then $T$ can be transformed into a simple positively oriented path by finitely many u-foldings.

Proof. By Lemma 3.15, $T$ can be transformed by finitely many $u$-foldings into a tree $T^{\prime}$ with $v_{0}^{\prime} \in V\left(T^{\prime}\right)$ corresponding to $v_{0}$, such that for any $v \in V\left(T^{\prime}\right)$ the unique reduced path $p_{v}$ from $v_{0}^{\prime}$ to $v$ is either positively oriented or negatively oriented. Now, to complete the proof it is enough to notice that any two finite simple positively oriented paths which have the same origin $w$ can be folded together into one simple positively oriented path with the same origin $w$. So, after finitely many $u$ foldings one transforms $T^{\prime}$ into two paths leading from $v_{0}^{\prime}$ - one positively oriented 
and another one negatively oriented. Their concatenation is a reduced positively oriented path.

Now, we return to $u$-components in a $(\mathbb{Z}[t], X)$-graph $\Gamma$.

Lemma 3.17. Let $\Gamma$ be a $(\mathbb{Z}[t], X)$-graph, $v \in V(\Gamma)$ and $C=C_{0 m p}(v)$ be finite. Then there exist a $(\mathbb{Z}[t], X)$-graph $\Delta$ obtained from $\Gamma$ by finitely many u-foldings such that $v^{\prime} \in V(\Delta)$ corresponds to $v$ and $C^{\prime}=C o m p_{u}\left(v^{\prime}\right)$ consists of a simple positively oriented path $P_{C^{\prime}}$, some vertices of which may be connected by single edges not in $P_{C^{\prime}}$.

Proof. Let $T$ be any spanning tree of $C$. Then, by Corollary 3.16 there exists a finite sequence $\left\{\phi_{1}, \ldots, \phi_{n}\right\}$ of $u$-foldings which transforms $T$ into a simple positively oriented path $P_{C^{\prime}}$. Observe that some vertices in $P_{C^{\prime}}$ can be connected by images of edges from $C-T$. Thus, $\Delta$ is the image of $\Gamma$ under $\left\{\phi_{1}, \ldots, \phi_{n}\right\}$.

We call $C^{\prime}$ from the lemma above a reduced $u$-component. Since $P_{C^{\prime}}$ is a simple path there exists a vertex $z_{C^{\prime}} \in V\left(P_{C^{\prime}}\right)$ such that $\operatorname{val}_{P_{C^{\prime}}}\left(z_{C^{\prime}}\right)=1$ and the only edge in $P_{C^{\prime}}$ which has $z_{C^{\prime}}$ as an origin is positive. We call $z_{C^{\prime}}$ a base-point of $C^{\prime}$.

Observe that because of arbitrary choice of a spanning tree for $C$ and arbitrary order of performing sequences of $u$-foldings it follows that the reduced $u$-component $C^{\prime}$ corresponding to $C$ is not unique.

Let $\Gamma$ be any $(\mathbb{Z}[t], X)$-graph, $v \in V(\Gamma)$ and let $C=C o m p_{u}(v)$ be a finite reduced $u$-component. Then there exists a simple positively oriented path $P_{C}$ in $C$ which originates from a base-point $z_{C}$ of $C$. Since $C$ is finite then there are finitely many edges $h_{1}, \ldots, h_{l}$ in $C-P_{C}$. Any $h_{i}$ connects two vertics in $P_{C}$ so there exists a unique reduced path $q_{i}$ in $P_{C}$ such that $o\left(h_{i}\right)=o\left(q_{i}\right), t\left(h_{i}\right)=t\left(q_{i}\right)$. Moreover, $h_{i} q_{i}^{-1}$ is a cycle in $C$, so $\mu\left(h_{i}\right) *{\overline{\mu\left(q_{i}\right)}}^{-1}=c \in H_{u}\left(z_{C}\right)$.

Now, let $p=e_{1} \cdots e_{n}$ be a reduced path in $C$. Suppose some of its edges $e_{i_{1}}, \ldots, e_{i_{k}}, i_{j} \in[1, n]$ belong to $C-P_{C}$. Then we construct another path $p^{\prime}$ in the following way: every edge $e_{i_{j}}$ is equal to some $h_{i_{j}}$ in the list of edges of $C-P_{C}$, so in $p$ we substitute $e_{i_{j}}$ by the path $q_{i_{j}}$. The resulting path $p^{\prime}$ may be not reduced, so we perform all possible reductions and obtain a reduced path $p^{\prime \prime}$ all edges of which belong to $P_{C}$. We have $o\left(p^{\prime \prime}\right)=o(p), t\left(p^{\prime \prime}\right)=t(p), \overline{\mu\left(p^{\prime \prime}\right)}=\overline{\mu(p)} * h, h \in H_{u}\left(z_{C}\right)$ by definition of $q_{i}$ for each $h_{i} \in C-P_{C}$. Finally, observe that $p^{\prime \prime}$ is uniquely defined for $p$ because it is a reduced subpath of $P_{C}$.

Hence, for any reduced path $p$ in $C$ there exists a unique reduced subpath $q$ of $P_{C}$ with the same endpoints as $p$ and such that $\overline{\mu(p)} * \overline{\mu(q)}^{-1} \in H_{u}\left(z_{C}\right)$. Further, we will use the notation $q=[p]$.

The converse is also true, that is, if $q$ is a reduced path in $P_{C}$ and $c \in H_{u}\left(z_{C}\right)$ then, since any element from $H_{u}\left(z_{C}\right)$ can be realized as a reduced label of some loop at $t(q)$ in $C$, there exists a reduced path $p$ in $C$ such that $o(p)=o(q), t(p)=$ $t(q), \overline{\mu(p)}=\overline{\mu(q)} * c$. Observe that $q$ is not unique with respect to permutation of cycles. 
The correspondence above shows that any finite reduced $u$-component $C$ in a $(\mathbb{Z}[t], X)$-graph is characterized completely by the pair $\left(P_{C}, H_{u}\left(z_{C}\right)\right)$.

If $C$ is finite then there exist finitely many subpaths $q_{1}, \ldots, q_{s}$ of $P_{C}$ such that for any path $p$ in $C,[p]=q_{i}$ for some $i \in[1, s]$. Moreover, let $P_{C}=f_{1} \cdots f_{m}$, where $o\left(f_{1}\right)=z_{C}$. Let $v_{0}=z_{C}, v_{i}=t\left(f_{i}\right), i \in[1, m]$ and let $p_{0}, p_{1}, \ldots, p_{m}$ be reduced subpaths of $P_{C}$ such that $o\left(p_{i}\right)=z_{C}, t\left(p_{i}\right)=v_{i}, i \in[0, m]$. It follows that all $p_{i}$ are positively oriented. Then for every reduced subpath $p_{i, j}$ of $P_{C}$ such that $o\left(p_{i, j}\right)=v_{i}, t\left(p_{i, j}\right)=v_{j}$ we have $\overline{\mu\left(p_{i, j}\right)}=\overline{\mu\left(p_{j}\right)} *{\overline{\mu\left(p_{i}\right)}}^{-1}$. By Lemma 3.11, $H_{u}\left(z_{C}\right)$ is finitely generated and is isomorphic to a subgroup of $\mathbb{Z}^{r}, r \in \mathbb{N}$. So if $p$ is a reduced path in $C$ such that $o(p)=v_{i}, t(p)=v_{j}$ then by definition of $[p]$ we have $\overline{\mu(p)}$ and $\overline{\mu([p])}=\overline{\mu\left(p_{i, j}\right)}=\overline{\mu\left(p_{j}\right)} *{\overline{\mu\left(p_{i}\right)}}^{-1}$ are in the same coset in $\mathbb{Z}^{r}$ by $H_{u}\left(z_{C}\right)$. That is, we can express the label of any reduced path in $C$ in terms of labels of $p_{i}, i \in[0, m]$ and elements from $H_{u}\left(z_{C}\right)$. We call a set of paths $p_{0}, p_{1}, \ldots, p_{m}$ a set of path representatives associated with $C$ and denote this set by $\operatorname{Rep}(C)$. The following result holds.

Lemma 3.18. Let $C$ be a finite reduced u-component in a $(\mathbb{Z}[t], X)$-graph $\Gamma, v \in$ $V(C)$ and let $\alpha \in \mathbb{Z}[t]$. If $\overline{\mu\left(p_{i}\right)} *{\overline{\mu\left(p_{j}\right)}}^{-1} \notin H_{u}\left(z_{C}\right)$ for any $p_{i}, p_{j} \in \operatorname{Rep}(C), i \neq j$ then either there exists a unique reduced path $p$ in $P_{C}$ such that $o(p)=v$ and $u^{\alpha} \in \overline{\mu(p)} * H_{u}\left(z_{C}\right)$, or there exists no path $q$ in $C$ with this property.

Proof. Suppose on the contrary that there exist two reduced paths $p, q$ in $P_{C}$ such that $o(p)=v, o(q)=v$ and $u^{\alpha}=\overline{\mu(p)} * h_{1}=\overline{\mu(q)} * h_{2}, h_{1}, h_{2} \in H_{u}\left(z_{C}\right)$. Then $\overline{\mu(p)} * \overline{\mu(q)}^{-1}=h_{1}^{-1} * h_{2} \in H_{u}\left(z_{C}\right)$.

On the other hand we have that $v=v_{i}, t(p)=v_{j}, t(q)=v_{k}, j \neq k$. So we have $\overline{\mu(p)}=\overline{\mu\left(p_{j}\right)} *{\overline{\mu\left(p_{i}\right)}}^{-1}, \overline{\mu(q)}=\overline{\mu\left(p_{k}\right)} *{\overline{\mu\left(p_{i}\right)}}^{-1}$. So $\overline{\mu(p)} * \overline{\mu(q)}^{-1}=\left(\overline{\mu\left(p_{j}\right)} *\right.$ \left.${\overline{\mu\left(p_{i}\right)}}^{-1}\right) *\left(\overline{\mu\left(p_{k}\right)} *{\overline{\mu\left(p_{i}\right)}}^{-1}\right)^{-1}=\overline{\mu\left(p_{j}\right)} *{\overline{\mu\left(p_{k}\right)}}^{-1} \in H_{u}\left(z_{C}\right)$ - contradiction.

If $C$ is reduced and $\operatorname{Rep}(C)$ satisfies the condition from Lemma 3.18 then we call $C$ a u-folded u-component.

Let $C$ be a finite reduced $u$-component in a $(\mathbb{Z}[t], X)$-graph $\Gamma$ which is not $u$-folded. That is, there exist two vertices $v_{i}, v_{j}, i<j$ in $P_{C}$ such that $\overline{\mu\left(p_{i}\right)} *{\overline{\mu\left(p_{j}\right)}}^{-1} \in H_{u}\left(z_{C}\right)$. Consider a graph $\Delta$ which is obtained from $\Gamma$ by identification of vertices $v_{i}, v_{j}$ in $C$ into one new vertex $v$. We call this operation a collapse of $v_{i}$ and $v_{j}$. The resulting $u$-component in $\Delta$ we denote by $C^{\prime}$. In fact, a collapse can be obtained as a finite sequence of $u$-foldings. Indeed, since $\overline{\mu\left(p_{i}\right)} *{\overline{\mu\left(p_{j}\right)}}^{-1}=h \in H_{u}\left(z_{C}\right)$ there exists a positive loop at $v_{i}$ with the label $h$. This means that if we add a single edge $e$ to $C$ so that $o(e)=t(e)=v_{i}, \mu(e)=h$ then $H_{u}\left(z_{C}\right)$ is not changed. Then we can apply a sequence of $j-i u$-foldings to $e$ and the subpath $q=e_{i+1} \cdots e_{j}$ of $P_{C}$ connecting $v_{i}$ and $v_{j}$. After these $u$-foldings are implemented, $v_{i}$ is identified with $v_{j}$ because $\overline{\mu(q)}=h$. Since $u$-foldings do not change $H_{u}\left(z_{C}\right)$ in $u$-components a collapse of $v_{i}$ and $v_{j}$ is a valid operation. Observe that $|V(C)|=\left|V\left(C^{\prime}\right)\right|+1$. If $C^{\prime}$ is not reduced so using finitely many $u$-foldings 
one can reduce it and since $u$-foldings do not increase the number of vertices for the resulting reduced $u$-component $C^{\prime \prime}$ we have $|V(C)|>\left|V\left(C^{\prime}\right)\right| \geq\left|V\left(C^{\prime \prime}\right)\right|$. Thus, the following result holds.

Lemma 3.19. Let $\Gamma$ be a $(\mathbb{Z}[t], X)$-graph which has finitely many u-components all of which are finite and reduced. Then there exists a partially folded $(\mathbb{Z}[t], X)$-graph $\Delta$ which is obtained from $\Gamma$ by finitely many u-foldings such that all its u-components are $u$-folded.

\section{4. $U$-Folded $(\mathbb{Z}[t], X)$-Graphs and Finitely Generated Subgroups of $F^{\mathbb{Z}[t]}$}

In this section we introduce the notion of $U$-folded $(\mathbb{Z}[t], X)$-graph. Using the notations developed in the previous section we show how one can transform an arbitrary $(\mathbb{Z}[t], X)$-graph into a $U$-folded one.

\subsection{Languages associated with $(\mathbb{Z}[t], X)$-graphs}

Recall that a graph labeled by letters from $X^{ \pm}$defines a language of words over $X^{ \pm}$. This language can be put into correspondence with a subgroup of a free group $F(X)$. In the present subsection we generalize this concept to $(\mathbb{Z}[t], X)$-graphs.

Definition 4.1. Let $\Gamma$ be a $(\mathbb{Z}[t], X)$-graph and let $v$ be a vertex of $\Gamma$. We define the language of $\Gamma$ with respect to $v$ to be

$$
L(\Gamma, v)=\{\overline{\mu(p)} \mid p \text { is a reduced path in } \Gamma \text { from } v \text { to } v\} .
$$

If $w$ belongs to $L(\Gamma, v)$, we will also sometimes say that $w$ is accepted by $(\Gamma, v)$ (or just by $\Gamma$ if $v$ is fixed).

The following result establishes a connection between $(\mathbb{Z}[t], X)$-graphs and subgroups in $F^{\mathbb{Z}[t]}$.

Lemma 4.2. Let $\Gamma$ be a finite $(\mathbb{Z}[t], X)$-graph and let $v \in V(\Gamma)$. Then $L(\Gamma, v)$ is a subgroup of $F^{\mathbb{Z}[t]}$.

Proof. Observe, at first, that $L(\Gamma, v)$ is a subset of $F^{\mathbb{Z}[t]}$ by definition.

Let $g_{1}, g_{2} \in L(\Gamma, v)$. Then there are reduced paths $p_{1}$ and $p_{2}$ from $v$ to $v$ in $\Gamma$ such that $\overline{\mu\left(p_{i}\right)}=g_{i}, i=1,2$.

The concatenation $q$ of $p_{1}$ and $p_{2}$ is a path in $\Gamma$ from $v$ to $v$ such that $\overline{\mu(q)}=$ $\overline{\mu\left(p_{1}\right)} * \overline{\mu\left(p_{2}\right)}$, but $q$ may not be reduced. Let $p$ be the reduced path obtained from $q$ by making all possible path reductions. This means that the label $\overline{\mu(p)}=\overline{\mu(q)}$ and $o(p)=t(p)=v$. Therefore $\overline{\mu\left(p_{1}\right)} * \overline{\mu\left(p_{2}\right)}=\overline{\mu(p)} \in L(\Gamma, v)$.

Thus, $g_{1} * g_{2} \in L(\Gamma, v)$ and $L(\Gamma, v)$ is closed under multiplication $*$ of infinite words.

It is easy to see that the inverse path $\left(p_{1}\right)^{-1}$ of $p_{1}$ is reduced and $\overline{\mu\left(p_{1}^{-1}\right)}=$ $\overline{\mu\left(p_{1}\right)^{-1}}={\overline{\mu\left(p_{1}\right)}}^{-1}$. This implies that $L(\Gamma, v)$ is closed under taking inverses. Also, obviously $\varepsilon \in L(\Gamma, v)$.

Thus, $L(\Gamma, v)$ is a subgroup of $F^{\mathbb{Z}[t]}$. 
In the previous section we introduced free foldings and $U$-foldings and now we show that these operations do not change the language associated with $\Gamma$.

Lemma 4.3. Let $\Gamma$ be a finite $(\mathbb{Z}[t], X)$-graph and let $v \in V(\Gamma)$. Let $\Delta_{1}$ be a $(\mathbb{Z}[t], X)$-graph obtained from $\Gamma$ by a single free folding and let $\Delta_{2}$ be a $(\mathbb{Z}[t], X)$ graph obtained from $\Gamma$ by a single u-folding for some $u \in U$, so that $v_{1} \in V\left(\Delta_{1}\right)$ and $v_{2} \in V\left(\Delta_{2}\right)$ correspond to $v$. Then

$$
L(\Gamma, v)=L\left(\Delta_{1}, v_{1}\right)=L\left(\Delta_{2}, v_{2}\right) .
$$

Proof. At first we prove $L(\Gamma, v)=L\left(\Delta_{1}, v_{1}\right)$. If $\Delta_{1}$ is obtained from $\Gamma$ by identification of edges $e_{1}, e_{2} \in \widehat{\Gamma}, \mu\left(e_{1}\right)=\mu\left(e_{2}\right)=x \in X^{ \pm 1}$ then the result follows from the proof of $\left[4\right.$, Lemma 3.4]. If $e_{1}, e_{2} \in \widehat{\Gamma}$ are labeled by the same exponent of some $u \in U$ then the free folding which identifies $e_{1}$ and $e_{2}$ is a $u$-folding and, thus, in this case, we reduced the proof of $L(\Gamma, v)=L\left(\Delta_{1}, v_{1}\right)$ to the proof of $L(\Gamma, v)=L\left(\Delta_{2}, v_{2}\right)$.

So let us prove $L(\Gamma, v)=L\left(\Delta_{2}, v_{2}\right)$.

Suppose $\Delta_{1}$ is obtained from $\Gamma$ by folding two edges $e_{1}, e_{2}$ in $\widehat{\Gamma}$ which have the same initial vertex $w$ and $\mu\left(e_{1}\right)=u^{\alpha}, \mu\left(e_{2}\right)=u^{\beta}$. Without loss of generality we can assume $\alpha \geq \beta>0$. Then $e_{1}$ becomes a path $h_{1} h_{2}$ in $\Delta_{2}$ such that $\mu\left(h_{1}\right)=$ $u^{\beta}, \mu\left(h_{2}\right)=u^{\alpha-\beta}$ and $h_{1}$ is identified with $e_{2}$ in $\Delta_{2}$ into an edge $h$.

Suppose $p$ is a reduced path in $\Gamma$ from $v$ to $v$, so that $\overline{\mu(p)} \in L(\Gamma, v)$. The image of $p$ in $\Delta_{1}$ by Lemma 3.13 is a path $p^{\prime}$ from $v_{2}$ to $v_{2}$ such that $\overline{\mu(p)}=\overline{\mu\left(p^{\prime}\right)}$. However, $p^{\prime}$ need not be reduced. Namely, $p^{\prime}$ is reduced if and only if $p$ does not contain any subpaths of the form $e_{2}^{-1} e_{1}$ or $e_{1}^{-1} e_{2}$. Let $p^{\prime \prime}$ be the path obtained from $p^{\prime}$ by performing all possible path reductions in $\Delta_{2}$. Then $\overline{\mu(p)}=\overline{\mu\left(p^{\prime}\right)}=\overline{\mu\left(p^{\prime \prime}\right)}$ and $\overline{\mu\left(p^{\prime \prime}\right)} \in L\left(\Delta_{2}, v_{2}\right)$. Thus, we have shown that $L(\Gamma, v) \subseteq L\left(\Gamma^{\prime}, v^{\prime}\right)$.

Suppose now that $p^{\prime}$ is an arbitrary reduced path in $\Delta_{2}$ from $v_{2}$ to $v_{2}$. We claim that there is a reduced path $p$ in $\Gamma$ from $v$ to $v$ such that $\overline{\mu(p)}=\overline{\mu\left(p^{\prime}\right)}$. We will construct this path explicitly.

(1) $\alpha=\beta$. In this case $h_{2}$ is an empty edge and $e_{1}, e_{2}$ are identified with the edge $h$ in $\Delta_{2}$.

The occurrences of $h^{ \pm 1}$ (if any) subdivide $p^{\prime}$ into a concatenation of the form:

$$
p^{\prime}=p_{0} f_{0} p_{1} f_{1} \cdots f_{k} p_{k+1},
$$

where $f_{i}=h^{ \pm 1}$ and the paths $p_{i}$ do not involve $h^{ \pm 1}$.

Suppose that for some $i$ we have $f_{i}=h$. Since $p_{i}$ and $p_{i+1}$ do not involve the edge $h$, they can also be considered as paths in $\Gamma$. Moreover, by the definition of $u$-folding, in the graph $\Gamma$ the terminal vertex of $p_{i}$ is joined with the initial vertex of $p_{i+1}$ by either the edge $e_{1}$ or $e_{2}$. We denote this edge by $d_{i}$ (so that $d_{i} \in\left\{e_{1}, e_{2}\right\}$ ). Note that now $p_{i} d_{i} p_{i+1}$ is a reduced path in $\Gamma$ with the same label as the path $p_{i} f_{i} p_{i+1}$ in $\Delta_{2}$.

Similarly, if for some $i$ we have $f_{i}=h^{-1}$, we can find $d_{i} \in\left\{e_{1}^{-1}, e_{2}^{-1}\right\}$ such that $p_{i} d_{i} p_{i+1}$ is a reduced path in $\Gamma$ with the same label as the path $p_{i} f_{i} p_{i+1}$ in $\Delta_{2}$. 
Then

$$
p=p_{0} d_{0} p_{1} \cdots d_{k} p_{k+1}
$$

is a reduced path in $\Gamma$ from $v$ to $v$ with the same label as $p^{\prime}$. Thus, $\overline{\mu\left(p^{\prime}\right)} \in L(\Gamma, v)$ and therefore $L\left(\Delta_{2}, v_{2}\right) \subseteq L(\Gamma, v)$.

(2) $\alpha>\beta$. This is a general case when $e_{1}$ becomes a path $h h_{2}$ and $e_{2}$ becomes the edge $h$ in $\Delta_{2}$. Observe that $\mu(h)=\mu\left(e_{2}\right), \mu\left(h_{2}\right)=\mu\left(e_{2}\right)^{-1} * \mu\left(e_{1}\right)$ and $o(h)=$ $o\left(e_{2}\right)=o\left(e_{1}\right), t(h)=t\left(e_{2}\right), o\left(h_{2}\right)=t(h)=t\left(e_{2}\right), t\left(h_{2}\right)=t\left(e_{1}\right)$ in $\Delta_{2}$.

Similarly to (1) we subdivide $p^{\prime}$ by the occurrences of $h^{ \pm 1}$ and $h_{2}^{ \pm 1}$ so that

$$
p^{\prime}=p_{0} f_{0} p_{1} f_{1} \cdots f_{k} p_{k+1},
$$

where $f_{i}=h^{ \pm 1}$ or $f_{i}=h_{2}^{ \pm 1}$ and the paths $p_{i}$ do not involve $h^{ \pm 1}, h_{2}^{ \pm 1}$.

Any entry of edge $h_{2}$ we substitute by a path $e_{2}^{-1} e_{1}$ and every entry of $h$ we substitute by $e_{2}$ so that $h_{2}^{-1}$ is substituted by $e_{1}^{-1} e_{2}$ and $h^{-1}$ by $e_{2}^{-1}$.

Suppose that for some $i$ we have $f_{i}=h$. Since $p_{i}$ and $p_{i+1}$ do not involve the edge $h$, they can also be considered as paths in $\Gamma$. We have $t\left(p_{i}\right)=o(h)=$ $o\left(e_{2}\right), o\left(p_{i+1}\right)=t(h)=t\left(e_{2}\right)$, so the substitution $h \rightarrow e_{2}$ is valid. If on the other hand for some $j$ we have $f_{j}=h_{2}$ then $t\left(p_{j}\right)=o\left(h_{2}\right)=t\left(e_{2}\right), o\left(p_{j+1}\right)=t\left(h_{2}\right)=t\left(e_{1}\right)$ and again the substitution $h_{2} \rightarrow e_{2}^{-1} e_{1}$ is valid. Finally, $\mu(h)=\mu\left(e_{2}\right), \mu\left(h_{2}\right)=$ $\mu\left(e_{2}\right)^{-1} * \mu\left(e_{1}\right)$ implies that after all possible substitutions in $p^{\prime}$ are made, we get the resulting path $p^{\prime \prime}$ in $\Gamma$ from $v$ to $v$ such that $\overline{\mu\left(p^{\prime \prime}\right)}=\overline{\mu\left(p^{\prime}\right)}$, but $p^{\prime \prime}$ may not be reduced. Let $p$ be the path obtained from $p^{\prime \prime}$ by performing all possible path reductions in $\Gamma$. Then $\overline{\mu(p)}=\overline{\mu\left(p^{\prime \prime}\right)}=\overline{\mu\left(p^{\prime}\right)}$ and $\overline{\mu(p)} \in L(\Gamma, v)$.

Hence, in both cases (1) and (2) above $L\left(\Delta_{2}, v_{2}\right) \subseteq L(\Gamma, v)$ which completes the proof.

Let $\Gamma$ be a $(\mathbb{Z}[t], X)$-graph and $p=e_{1} \cdots e_{k}$ be a reduced path in $\Gamma$. Let $g \in$ $G_{n+1}-G_{n}$ and let

$$
\pi(g)=\pi\left(h_{1}\right) u^{\beta_{1}} \pi\left(h_{2}\right) \cdots u^{\beta_{l}} \pi\left(h_{l+1}\right),
$$

be the standard decomposition of $g$, where $u=\max \{U(g)\}$. We write

$$
\mu(p)=\pi(g)
$$

if $p$ can be subdivided into subpaths

$$
p=p_{1} d_{1} p_{2} \cdots d_{l} p_{l+1},
$$

where $d_{i}$ is a path in some $u$-component of $\Gamma$ and $p_{i}$ is a path in $\Gamma$ which does not contain edges labeled by $u^{\alpha}, \alpha \in \mathbb{Z}[t]$, so that $\overline{\mu\left(d_{i}\right)}=u^{\beta_{i}}, i \in[1, l]$ and each equality $\mu\left(p_{i}\right)=\pi\left(h_{i}\right), i \in[1, l+1]$ is defined inductively in the same way. Observe that if $g=x_{1} \cdots x_{r} \in F$ then $\mu(p)=\pi(g)$ if $k=r$ and $\mu\left(e_{i}\right)=x_{i}$ for every $i \in[1, k]$.

It follows immediately that if $\mu(p)=\pi(g)$ for some $g \in F^{\mathbb{Z}[t]}$ then $p$ is label reduced.

Observe that if $\Gamma$ is a $(\mathbb{Z}[t], X)$-graph and $v \in V(\Gamma)$ then it follows from Lemma 4.2 that any element of $L(\Gamma, v)$ is an infinite word in $F^{\mathbb{Z}[t]}$ and, thus, has 
the standard decomposition $\pi(g)$ but it might be impossible to find a label reduced loop $p$ at $v$ in $\Gamma$ such that $\mu(p)=\pi(g)$. The main result of the following subsection is that if $\Gamma$ is finite and has some particular properties then it is always possible to find such a path $p$ and $p$ is unique in some sense. Moreover, it will be shown how the required properties can be obtained in $\Gamma$ using free foldings and $U$-foldings.

\section{2. $U$-folded $(\mathbb{Z}[t], X)$-graphs}

In this subsection we obtain the main technical result of the paper.

Let $\Gamma$ be a finite $(\mathbb{Z}[t], X)$-graph. Since $\Gamma$ is finite there exist only finitely many edges with labels $u_{e}^{\alpha}, u_{e} \in U, \alpha \in \mathbb{Z}[t]$. Thus, there exists $K \in \mathbb{N}$ such that for any $e \in E(\Gamma)$ with $\mu(e)=u_{e}^{\alpha}$ it follows that $u_{e} \in U_{j_{e}}, j_{e} \leq K$. It is easy to see that for each fixed $j_{e}$ using $u_{e}$-foldings described in Sec. 3 one can transform all $u_{e}$-components of $\Gamma$ into $u_{e}$-folded components. However, because of the nature of the sets $U_{j_{e}}$, edges of $\Gamma$ belong to different levels (we introduce the precise definition of a level below) and $U$-foldings do not deal with interactions between these levels. Moreover, $u$-foldings applied to some $u$-component can affect $w$-components $u \neq w$. So, one needs some definite procedure which "folds" $\Gamma$ level by level.

Since $\Gamma$ is finite the set of elements $u \in U$ such that there exists an edge $e$ in $\Gamma$ labeled by $u^{\alpha}, \alpha \in \mathbb{Z}[t]$ is finite and the order from $U$ is induced on it. Thus one can associate with $\Gamma$ an ordered set $U(\Gamma)=\left\{u_{1}, \ldots, u_{N}\right\}$, where $N>0, u_{i} \in U, i \in$ $[1, N]$ and $u_{i}<u_{j}$ if $i<j$. Observe that $U(\Gamma)$ can be empty in case when all edges of $\Gamma$ are labeled by letters from $X^{ \pm}$.

Definition 4.4. Let $\Gamma$ be a finite $(\mathbb{Z}[t], X)$-graph and let $u_{i} \in U(\Gamma)$ be fixed. Let $\Gamma(i)$ be a subgraph of $\Gamma$ which consists only of edges $e \in E(\Gamma)$ such that either $\mu(e)=w \in X^{ \pm}$or $\mu(e)=u_{j}^{\alpha}, \alpha \in \mathbb{Z}[t], j \leq i$. We call $\Gamma(i)$ an $i$-level graph of $\Gamma$ (by 0 -level graph we understand a subgraph of $\Gamma$ which consists only of edges with labels from $X$ ) and say that $\Gamma$ has level $n$ denoted by $l(\Gamma)$ if $n$ is the minimal natural number for which $\Gamma=\Gamma(n)$.

Observe that $\Gamma(i)$ may be not connected for some $i<l(\Gamma)$, but still one can apply to $\Gamma(i)$ free foldings and $u$-foldings $u \in U(\Gamma)$.

Definition 4.5. Let $\Gamma$ be a finite connected $(\mathbb{Z}[t], X)$-graph and let $U(\Gamma)=$ $\left\{u_{1}, \ldots, u_{l(\Gamma)}\right\}$. If $u_{n} \in U(\Gamma)$ and $C$ is a $u_{n}$-component of $\Gamma$ then

(1) a path $p$ in $\Gamma(n-1)$ is called $\left(u_{n}, \delta\right)$-irregular of type $I$ if $o(p) \in V(C), \overline{\mu(p)}=w$, $w=u_{n}^{\delta} \circ c_{p}, \delta \in\{1,-1\}$,

(2) a path $p$ in $\Gamma(n)$ is called $\left(u_{n}, \delta\right)$-irregular of type $I I$ if $p=p_{\Gamma(n-1)} p_{\Gamma(n)}, o(p) \in$ $V(C), p_{\Gamma(n-1)} \in \Gamma(n-1), \overline{\mu\left(p_{\Gamma(n-1)}\right)}=w_{1}, \overline{\mu\left(p_{\Gamma(n)}\right)}=w_{2} \circ c=u_{n}^{\gamma} \circ c_{1}, \gamma \in$ $\mathbb{Z}[t]-\mathbb{Z}, u_{n}^{\delta}=w_{1} \circ w_{2}, w_{2} \neq \varepsilon, \delta \in\{1,-1\}$,

(3) a path $p$ in $\Gamma(n-1)$ is called $\left(u_{n}, \delta\right)$-irregular of type III if $o(p) \in V(C), \overline{\mu(p)}=$ $w_{1}(p), u_{n}^{\delta}=w_{1}(p) \circ w_{2}(p), w_{2}(p) \neq \varepsilon, \delta \in\{1,-1\}$ and there exists a path $p^{\prime}$ in $C$ such that $o\left(p^{\prime}\right)=o(p), \overline{\mu\left(p^{\prime}\right)}=u_{n}^{\gamma}, \gamma \in \mathbb{Z}[t], \gamma \delta>0$. 
Definition 4.6. Let $\Gamma$ be a finite connected $(\mathbb{Z}[t], X)$-graph and let $U(\Gamma)=$ $\left\{u_{1}, \ldots, u_{l(\Gamma)}\right\}$. If $u_{n} \in U(\Gamma)$ and $C$ is a $u_{n}$-component of $\Gamma$ then

(1) a $\left(u_{n}, \delta\right)$-irregular path $p$ of type I originating from $v$ is doubled if there exists a label reduced path $q=q_{1} q_{2}$ in $\Delta(n-1)$ such that $o(q)=v, t(q)=t\left(q_{2}\right)=$ $t(p), \overline{\mu(q)}=\overline{\mu(p)}, \mu\left(q_{1}\right)=\pi\left(u_{n}\right)^{\delta}, t\left(q_{1}\right) \in V(C)$,

(2) a $\left(u_{n}, \delta\right)$-irregular path $p$ of type II originating from $v$ is doubled if there exists a label reduced path $q=q_{1} q_{2}$ in $\Delta(n)$ such that $o(q)=v, t(q)=t\left(q_{2}\right)=$ $t(p), \overline{\mu(q)}=\overline{\mu(p)}, \mu\left(q_{1}\right)=\pi\left(u_{n}\right)^{\delta}, t\left(q_{1}\right) \in V(C)$,

(3) a $\left(u_{n}, \delta\right)$-irregular path $p$ of type III originating from $v$ is doubled if there exists a label reduced path $q=q_{1} q_{2}$ in $\Delta(n-1)$ such that $o(q)=v, t(q)=t\left(q_{2}\right)=$ $t(p), \overline{\mu(q)}=\overline{\mu(p)}, \mu\left(q_{1}\right)=\pi\left(w_{1}(p)\right), \mu\left(q_{2}\right)=\pi\left(w_{2}(p)\right), t(q) \in V(C)$.

Definition 4.7. Let $\Delta$ be a finite connected $(\mathbb{Z}[t], X)$-graph and let $U(\Delta)=$ $\left\{u_{1}, \ldots, u_{l(\Delta)}\right\} . \Delta$ is called $U$-folded if for any $u_{n} \in U(\Delta)$ the following conditions are satisfied:

(1) $\Delta$ is partially folded;

(2) all $u_{n}$-components of $\Delta$ are $u_{n}$-folded and isolated in the sense that there exists no reduced path $p$ with $\overline{\mu(p)}=u_{n}^{k}, k \in \mathbb{Z}$ in $\Delta(n-1)$ such that $p$ connects two different $u_{n}$-components of $\Delta$;

(3) if $C$ is a $u_{n}$-component of $\Delta, e \in E\left(P_{C}\right)$ and $\mu(e)=u_{n}^{k}, k \in \mathbb{Z}$ then there exists a unique label reduced path $p$ in $\Delta(n-1)$ such that $o(p)=o(e), t(p)=$ $t(e), \mu(p)=\pi\left(u_{n}\right)^{k}$;

(4) if $C$ is a $u_{n}$-component of $\Delta$ and $v \in V(C) \cap V(\Delta(n-1))$ then there exists a unique label reduced path $p$ in $\Delta(n-1)$ such that $o(p)=t(p)=v, \mu(p)=$ $\pi\left(u_{n}\right)^{k}, k \in \mathbb{Z}$ and $H_{u_{n}}\left(z_{C}\right) \cap\left\langle u_{n}\right\rangle=\left\langle u_{n}^{k}\right\rangle ;$

(5) if $C$ is a $u_{n}$-component of $\Delta$ and $v_{1}, v_{2} \in V(C)$ are connected by a reduced subpath $p$ of $P_{C}$ then either $p$ consists only of edges labeled by finite exponents of $u_{n}$ or there exists no number $k_{p} \in \mathbb{Z}$ such that $\overline{\mu(p)} * u^{-k_{p}} \in H_{u_{n}}\left(z_{C}\right)$;

(6) for any $u_{n}$-component $C$ of $\Delta$ and its distinct vertices $v_{1}, v_{2}$ which are connected by a reduced subpath $p$ of $P_{C}$ with $o(p)=v_{1}, t(p)=v_{2}$ there exists no reduced path $r$ in $\Delta(n-1)$ such that $o(r)=v_{1}, t(r)=v_{2}, \overline{\mu(r)}=u_{n}^{k}, k \in \mathbb{Z}$ and $\overline{\mu(p)} * \overline{\mu(r)}^{-1} \notin H_{u_{n}}\left(v_{1}\right)$;

(7) for any $u_{n}$-component $C$ of $\Delta, v \in V(C)$ and a reduced path $p$ in $\Delta(n-1)$ such that $o(p)=v, \overline{\mu(p)}=u_{n}^{k}, k \in \mathbb{Z}$ it follows that $t(p) \in V(C)$;

(8) for any $u_{n}$-component $C$ of $\Delta$ and its vertex $v$ any $\left(u_{n}, \delta\right)$-irregular path $p$ of any type originating from $v$ is doubled in $\Delta(n)$.

Remark 4.8. Observe that in (3) and (4) by uniqueness we understand uniqueness with respect to $P_{C}$, where $C$ is any $u$-component $u \in U(\Delta)$, that is, a path is unique if we disregard the order of edges in $C-P_{C}$. This is justified in view of Lemma 3.18. 
The definition above is very technical and it is not clear why we need all the properties listed. However, using them in the proof of the next proposition we are able to show the most essential properties of $U$-folded graphs.

Proposition 4.9. Let $\Delta$ be a $U$-folded graph. Then the following hold:

(1) for any reduced path $p$ in $\Delta$ with $\overline{\mu(p)}=w$ there exists a unique label reduced path $q$ such that $o(q)=o(p), t(q)=t(p), \mu(q)=\pi(w)$;

(2) for the standard decomposition $\pi(g)$ of any $g \in F^{\mathbb{Z}[t]}$ and any $v \in V(\Delta)$, either there exists a unique label reduced path $p$ in $\Delta$ starting at $v$ such that $\mu(p)=\pi(g)$ or for any path $q$ in $\Delta$ starting at $v$ it follows that $\overline{\mu(q)} \neq g$.

Proof. We take advantage of the level structure of $\Delta$ - the proof is conducted by the induction on $l(\Delta)$. If $l(\Delta)=0$, that is, all edges in $\Delta$ are labeled by letters from $X^{ \pm}$, then $\Delta$ is partially folded and the statement of the proposition follows from [4, Lemma 3.9].

Assume that the statement of the theorem holds for any graph of level $n-1$. Let $l(\Delta)=n$ and let $U(\Delta)=\left\{u_{1}, \ldots, u_{l(\Delta)}\right\}$.

(1) Let $p$ be a path in $\Delta$ with $\overline{\mu(p)}=w \cdot \mu(p)$ belongs to the alphabet $X^{ \pm} \cup\left\{u^{\alpha} \mid\right.$ $\left.u \in U(\Delta), u \leq u_{n}, \alpha \in \mathbb{Z}[t]\right\}$ so let us subdivide $p$ in the following way:

$$
p=p_{1} d_{1} p_{2} d_{2} \cdots p_{m} d_{m} p_{m+1},
$$

where every $p_{i}, i \in[1, m+1]$ does not contain edges labeled by $u_{n}^{\alpha}, \alpha \in \mathbb{Z}[t]$, so that we can assume every $p_{i}$ to be a path in $\Delta(n-1)$, and every $d_{i}, i \in[1, m]$ contains only edges labeled by $u_{n}^{\alpha}, \alpha \in \mathbb{Z}[t]$.

Take any $p_{i}$. By induction there exists a unique label reduced path $q_{i}$ such that $o\left(p_{i}\right)=o\left(q_{i}\right), t\left(p_{i}\right)=t\left(q_{i}\right), \mu\left(q_{i}\right)=\pi\left(h_{i}\right)$, where $\overline{\mu\left(p_{i}\right)}=h_{i}$. Observe that since $t\left(p_{i}\right)$ belongs to some $u_{n}$-component $C_{i}$ and $d_{i}$ is a path composed only of edges labeled by exponents of $u_{n}$ then $d_{i}$ is a path in $C_{i}$ such that $\overline{\mu\left(d_{i}\right)}=u_{n}^{\alpha_{i}}$. By Lemma 3.18 there exists a unique path $c_{i}$ in $P_{C_{i}}$ such that $o\left(c_{i}\right)=o\left(d_{i}\right)$ and $u_{n}^{\alpha_{i}} \in \overline{\mu\left(c_{i}\right)} * H_{u_{n}}\left(o\left(d_{i}\right)\right)$. We have $t\left(d_{i}\right)=t\left(c_{i}\right)$. Indeed, if $d_{i}$ leads to some other vertex $v \in P_{C_{i}}$ then there exists a unique positively oriented subpath $p_{v}$ of $P_{C_{i}}$ from $o\left(d_{i}\right)$ to $v$ and we have a loop $p_{v} d_{i}^{-1}$, so $\overline{\mu\left(p_{v}\right)} * u_{n}^{-\alpha_{i}} \in H_{u_{n}}\left(o\left(d_{i}\right)\right)$. Thus, $u_{n}^{\alpha_{i}}=\overline{\mu\left(p_{v}\right)} * h_{i}, h_{i} \in H_{u_{n}}\left(o\left(d_{i}\right)\right)$ and $\overline{\mu\left(p_{v}\right)} \in \overline{\mu\left(c_{i}\right)} * H_{u_{n}}\left(o\left(d_{i}\right)\right)$ which is impossible unless $p_{v}=c_{i}$, because $C_{i}$ is $u_{n}$-reduced. So $t\left(d_{i}\right)=t\left(c_{i}\right)=o\left(p_{i+1}\right)$.

Thus, we obtain a reduced path $r$ as the following concatenation:

$$
q^{\prime}=q_{1} c_{1} q_{2} c_{2} \cdots q_{m} c_{m} q_{m+1}
$$

where $o\left(q_{i}\right)=o\left(p_{i}\right), t\left(q_{i}\right)=t\left(p_{i}\right), o\left(c_{i}\right)=o\left(d_{i}\right), t\left(c_{i}\right)=t\left(d_{i}\right), \overline{\mu\left(q_{i}\right)}=\overline{\mu\left(p_{i}\right)}$, $\overline{\mu\left(c_{i}\right)}=\overline{\mu\left(d_{i}\right)}=u_{n}^{\alpha_{i}}, i \in[1, m+1]$ and $o\left(q^{\prime}\right)=o(p), t\left(q^{\prime}\right)=t(p), \overline{\mu\left(q^{\prime}\right)}=\overline{\mu(p)}$ and all $q_{i}, c_{i}$ are label reduced.

However, $q^{\prime}$ may be not label reduced, that is, there can be a cancellation in $\overline{\mu\left(c_{i}\right)} * \overline{\mu\left(q_{i+1}\right)}$. Without loss of generality we can assume $\alpha_{i} \gg 0$ (if not then by the 
property (3) of $U$-folded graphs we can assume $p_{i} c_{i} p_{i+1}$ to be a path in $\Delta(n-1)$ and everything follows by induction for $\left.p_{i} c_{i} p_{i+1}\right)$.

Suppose $\overline{\mu\left(c_{i}\right)} * \overline{\mu\left(q_{i+1}\right)} \neq \overline{\mu\left(c_{i}\right)} \circ \overline{\mu\left(q_{i+1}\right)}$. Then $\overline{\mu\left(q_{i+1}\right)}$ contains an initial subword $u_{n}^{k} \circ g_{1}, k<0, u_{n}^{-1}=g_{1} \circ g_{2} . \Delta(n-1)$ is $U$-folded and the induction hypothesis holds for it, hence it follows that there exists a path $r_{i+1}=z_{i+1} f_{i+1}$ such that $o\left(r_{i+1}\right)=o\left(q_{i+1}\right), t\left(r_{i+1}\right)=t\left(q_{i+1}\right), o\left(z_{i+1}\right)=v_{1} \in V\left(C_{i}\right), \mu\left(z_{i+1}\right)=\pi\left(u_{n}\right)^{k} \pi\left(g_{1}\right)$ and it can be continued by a path $z^{\prime}$ to some vertex $v_{2} \in V\left(C_{i}\right)$ so that $z_{i+1} z^{\prime}$ is a label reduced path from $v_{1}$ to $v_{2}$. Let $w_{i+1}$ be the path in $P_{C_{i}}$ such that $o\left(w_{i+1}\right)=v_{1}, t\left(w_{i+1}\right)=v_{2}$. Then we have that concatenation $w_{i+1} z^{\prime-1}$ is a path from $t\left(c_{i}\right)=o\left(q_{i+1}\right)=o\left(z_{i+1}\right)$ to $t\left(z_{i+1}\right)=o\left(f_{i+1}\right)$ and $\overline{\mu\left(w_{i+1} z^{\prime-1}\right)}=\overline{\mu\left(z_{i+1}\right)}=$ $u_{n}^{k} \circ g_{1}$. So we can substitute $q_{i+1}$ by $w_{i+1} z^{\prime-1} f_{i+1}$ in $q$. Observe that $\overline{\mu\left(z^{\prime-1}\right)}=g_{2}^{-1}$ and $z^{\prime-1} f_{i+1}$ is label reduced otherwise $u_{n}^{k} \circ g_{1}$ is not the maximal initial subword of $\overline{\mu\left(q_{i+1}\right)}$ which cancels in $\overline{\mu\left(c_{i}\right)} * \overline{\mu\left(q_{i+1}\right)}$. Also, $u_{n} * g_{2}^{-1}=u_{n} \circ g_{2}^{-1}$.

Thus, $c_{i} q_{i+1}$ becomes $c_{i} w_{i+1} z^{\prime-1} f_{i+1}$. We can find a unique label reduced path $b_{i}$ in $C_{i}$ which corresponds to $c_{i} w_{i+1}$ and then $b_{i} z^{\prime-1} f_{i+1}$ is label reduced.

If $\overline{\mu\left(q_{i}\right)} * \overline{\mu\left(c_{i}\right)} \neq \overline{\mu\left(q_{i}\right)} \circ \overline{\mu\left(c_{i}\right)}$ then we can substitute $q_{i}$ by $f_{i} z^{\prime \prime-1} w_{i}$ and using the same argument as above show that we obtain a label reduced path $f_{i} z^{\prime \prime}-1 b_{i}$ which corresponds to $q_{i} c_{i}$.

After finitely many such substitutions we get a label reduced path $q$ such that $o(q)=o(p), t(q)=t(p), \overline{\mu(q)}=\overline{\mu(p)}=w$. Finally, using the property (8) one can construct a unique label reduced path $q^{\prime}$ such that $o\left(q^{\prime}\right)=o(p), t\left(q^{\prime}\right)=$ $t(p), \mu\left(q^{\prime}\right)=\pi(w)$.

(2) Let $g \in F^{\mathbb{Z}[t]}$ be such that

$$
\pi(g)=\pi\left(h_{1}\right) u^{\alpha_{1}} \pi\left(h_{2}\right) \cdots u^{\alpha_{m}} \pi\left(h_{m+1}\right) .
$$

If $u<u_{n}$ then (2) follows by induction. Suppose $u=u_{n}$. By induction either there exists a unique label reduced path $p_{1}$ in $\Delta(n-1)$ for $h_{1}$ originating from $v \in \Delta(n)$ such that $\mu\left(p_{1}\right)=\pi\left(h_{1}\right)$ or for any path $q_{1}$ in $\Delta_{7}$ originating from $v$ it follows that $\overline{\mu\left(q_{1}\right)} \neq h_{1}$. In the latter case there exists no path $p$ for $g$ such that $\overline{\mu(p)}=g$, because if it exists then by (1) there exists a unique path $q$ such that $o(q)=o(p), t(q)=t(p), \mu(q)=\pi(g)$. But $q$ contains an initial subpath $q_{1}$ originating from $v$ with the label $\mu\left(q_{1}\right)=\pi\left(h_{1}\right)$ - a contradiction. In the former case we continue with $u^{\alpha_{1}}$. Since $t\left(p_{1}\right)$ belongs to some $u$-component $C_{1}$ of $\Delta(n)$ then by Lemma 3.18 either there exists a unique path $c_{1}$ in $P_{C_{1}}$ such that $o\left(c_{i}\right)=t\left(p_{1}\right)$ and $u^{\alpha_{i}} \in \overline{\mu\left(c_{1}\right)} * H_{u}\left(o\left(c_{i}\right)\right)$ or there exists no continuation of $p_{1}$ in $C_{1}$ which is labeled by $u^{\alpha_{i}}$. Again, if this continuation does not exist then there exists no required path for $g$, if it does exist then we continue. After at most $2 m+1$ number of steps we get the required result. This completes the proof of (2) and the proposition.

In order to prove the main technical result of this section we need several lemmas. 
Lemma 4.10. Let $\Gamma$ be a finite $U$-folded $(\mathbb{Z}[t], X)$-graph such that $U(\Delta)=$ $\left\{u_{1}, \ldots, u_{l(\Delta)}\right\}$ and let $u \in U$ be such that $u>u_{l(\Delta)}$. If $v \in V(\Gamma)$ and there exists a path $p$ in $\Gamma$ such that $o(p)=v, \mu(p)=\pi\left(u^{ \pm k}\right), k \in \mathbb{N}$ then there exists a path $q$ in $\Gamma$ such that $o(q)=v, \mu(q)=\pi\left(u^{ \pm 1}\right)$.

Proof. By the choice of $U$ we have $\pi\left(u^{2}\right)=\pi(u) \pi(u)=\pi(u)^{2}$. Hence, it implies that a path in $\Gamma$ originating from $v$ and labeled by $\pi\left(u^{k}\right), k \in \mathbb{N}$ contains an initial subpath labeled by $\pi(u)$.

On the other hand, let $p$ be a path in $\Gamma$ such that $o(p)=v, \mu(p)=\pi\left(u^{-k}\right)$, $k \in \mathbb{N}$. Thus, we have a path $p^{-1}$ originating from $t(p)$ such that $\overline{\mu\left(p^{-1}\right)}=u^{k}$. Since $\Gamma$ is $U$-folded it follows that there exists a path $r$ in $\Gamma$ such that $o(r)=$ $t(p), \mu(r)=\pi\left(u^{k}\right)$. By the choice of $U$ we have $\pi\left(u^{k}\right)=\pi(u)^{k}$ and hence $r$ contains a terminal subpath $r_{1}$ such that $t\left(r_{1}\right)=v, \mu\left(r_{1}\right)=\pi(u)$. Thus, for $r_{1}^{-1}$ we have $o\left(r_{1}^{-1}\right)=v, \overline{\mu\left(r_{1}^{-1}\right)}=u^{-1}$. Finally, since $\Gamma$ is $U$-folded there exists a path $q$ such that $o(q)=v, \mu(q)=\pi\left(u^{-1}\right)$.

Lemma 4.11. Let $\Gamma$ be a finite $(\mathbb{Z}[t], X)$-graph such that $U(\Gamma)=\left\{u_{1}, \ldots, u_{l(\Gamma)}\right\}$. Let $u_{n} \in U(\Gamma), C$ be a $u_{n}$-component of $\Gamma, v \in V(C)$. Suppose $\Gamma(n-1)$ is $U$-folded. If there exists a path $p$ in $\Gamma(n-1)$ such that $o(p)=v, \overline{\mu(p)}=u_{n}^{k} \circ c, k \in \mathbb{Z}$ then there exists a path $r=r_{1} r_{2}$ in $\Gamma(n)$ such that $o(r)=o(p), t(r)=t(p), \overline{\mu(r)}=$ $\overline{\mu(p)}, r_{1} \in \Gamma(n-1), \mu\left(r_{1}\right)=\pi\left(u_{n}\right)^{l},|k|=|l|+1, t\left(p_{1}\right) \in V(C)$ and $r_{2}$ is a $\left(u_{n}, \delta\right)$-irregular path of type I or II, where $\delta k>0$.

Proof. Without loss of generality we can assume that $k>0$ and also that $u_{n-1}=$ $\max \left\{U\left(u_{n}\right)\right\}$. Hence

$$
u_{n}=h_{1} \circ u_{n-1}^{\alpha_{1}} \circ h_{2} \circ \cdots \circ u_{n-1}^{\alpha_{m}} \circ h_{m+1},
$$

where $m \geq 1$ and

$$
\pi\left(u_{n}\right)=\pi\left(h_{1}\right) u_{n-1}^{\alpha_{1}} \pi\left(h_{2}\right) \cdots u_{n-1}^{\alpha_{m}} \pi\left(h_{m+1}\right) .
$$

Since $\pi\left(u_{n} \circ u_{n}\right)=\pi\left(u_{n}\right) \pi\left(u_{n}\right)$ then

$$
\begin{aligned}
\pi\left(u_{n}^{k} \circ c\right)= & \left(\pi\left(h_{1}\right) u_{n-1}^{\alpha_{1}} \pi\left(h_{2}\right) \cdots u_{n-1}^{\alpha_{m}} \pi\left(h_{m+1}\right)\right)^{k-1} \pi\left(h_{1}\right) u_{n-1}^{\alpha_{1}} \\
& \times \pi\left(h_{2}\right) \cdots \pi\left(u_{n-1}^{\alpha_{m}} \circ h_{m+1} \circ c\right) .
\end{aligned}
$$

Since $\Gamma(n-1)$ is $U$-folded then by Proposition 4.9 there exists a path $r$ in $\Gamma(n-1)$ such that $o(r)=o(p), t(r)=t(p), \mu(r)=\pi\left(u_{n}^{k} \circ c\right)$. Hence, there exists an initial subpath $r_{1}$ of $r$ such that $t\left(r_{1}\right) \in V(C)$ and

$$
\mu\left(r_{1}\right)=\left(\pi\left(h_{1}\right) u_{n-1}^{\alpha_{1}} \pi\left(h_{2}\right) \cdots u_{n-1}^{\alpha_{m}} \pi\left(h_{m+1}\right)\right)^{k-1},
$$

and the initial subpath $r_{2}$ of $r$ such that $r=r_{1} r_{2}$ is a $\left(u_{n}, \delta\right)$-irregular path of type I, where $\delta k>0$. 
Lemma 4.12. Let $\Gamma$ be a finite $(\mathbb{Z}[t], X)$-graph such that $U(\Gamma)=\left\{u_{1}, \ldots, u_{l(\Gamma)}\right\}$. Let $u_{n} \in U(\Gamma), C$ be a $u_{n}$-component of $\Gamma, v \in V(C)$. Suppose

(1) $\Gamma(n-1)$ is $U$-folded,

(2) $\Gamma(n)$ satisfies the conditions (1) $-(7)$

(3) there exists a path $p$ in $\Gamma(n)$ such that $o(p)=v, \overline{\mu(p)}=u_{n}^{k} \circ c, k \in \mathbb{Z}$ and $c$ does not have $u_{n}^{ \pm 1}$ as an initial segment.

Then there exists a path $r=r_{1} r_{2}$ in $\Gamma(n)$ such that $o(r)=o(p), t(r)=t(p), \overline{\mu(r)}=$ $\overline{\mu(p)}, r_{1} \in \Gamma(n-1)$ and one of the following holds:

(1) $\overline{\mu\left(r_{1}\right)}=u_{n}^{k} \circ c_{1}$,

(2) $\mu\left(r_{1}\right)=\pi\left(u_{n}\right)^{l},|l|=|k|-1, t\left(r_{1}\right) \in V(C)$ and $r_{2}$ is a $\left(u_{n}, \delta\right)$-irregular path of type II, where $\delta k>0$.

Proof. Without loss of generality we can assume that $k>0$.

Let $p_{1}$ be the maximal initial subpath of $p$ which does not contain edges labeled by $u_{n}^{\alpha}, \alpha \in \mathbb{Z}[t]-\mathbb{Z}$. Since $\Gamma(n)$ satisfies the conditions (1)-(7) then we can assume that $p_{1} \in \Gamma(n-1)$. If $\overline{\mu\left(p_{1}\right)}=u_{n}^{k} \circ c_{1}$ then we are done. Suppose on the contrary that $\overline{\mu\left(p_{1}\right)}=u_{n}^{l} \circ w_{1}$ and $l<k$. Hence, $u_{n}=w_{1} \circ w_{2}$. If $l<k-1$ then we have a contradiction since we obtain $w_{1} \circ w_{2}=w_{2} \circ w_{1}$. Thus, $l=k-1$.

Since $\Gamma(n-1)$ is $U$-folded then by Proposition 4.9 there exists a path $q$ in $\Gamma(n-1)$ such that $o(q)=o\left(p_{1}\right), t(q)=t\left(p_{1}\right), \mu(q)=\pi\left(u_{n}^{l} \circ w_{1}\right)$. On the other hand we have

$$
\pi\left(u_{n}^{l} \circ w_{1}\right)=\pi\left(u_{n}^{l}\right) \pi\left(w_{1}\right),
$$

so $q$ can be subdivided as $q=q_{1} q_{2}$ so that $\mu\left(q_{1}\right)=\pi\left(u_{n}^{l}\right)=\pi\left(u_{n}\right)^{l}, \mu\left(q_{2}\right)=\pi\left(w_{1}\right)$. Finally, if $p_{2}$ is such that $p=p_{1} p_{2}$ then we set $r_{1}=q_{1}$ and $r_{2}=q_{2} p_{2}$. Observe that $t\left(r_{1}\right) \in V(C)$ because $\Gamma(n)$ satisfies the conditions $(1)-(7)$ and $r_{2}$ is a $\left(u_{n}, \delta\right)$ irregular path of type II, where $\delta=1$.

Proposition 4.13. Let $\Gamma$ be a finite connected $(\mathbb{Z}[t], X)$-graph. Then there exists a $U$-folded $(\mathbb{Z}[t], X)$-graph $\Delta$ which is obtained from $\Gamma$ by a finite sequence of free and $u$-foldings. Moreover $\Delta$ can be found effectively.

Proof. To prove the proposition we use the induction on $l(\Gamma)$. If $l(\Gamma)=0$, that is, all edges in $\Gamma$ are labeled by letters from $X^{ \pm}$, then by Lemma 3.6 using finitely many free foldings one can obtain from $\Gamma$ a partially folded $(\mathbb{Z}[t], X)$-graph $\Delta$ which satisfies all the conditions (1)-(8) since it does not contain $u$-components.

Assume that the statement of the theorem holds for any graph of level $n-1$. Let $l(\Gamma)=n$ and let $U(\Gamma)=\left\{u_{1}, \ldots, u_{n}\right\}$.

We construct a $(\mathbb{Z}[t], X)$-graph $\Delta$ from $\Gamma$ in several steps which are described in the series of claims below.

Claim 4.14. Let $\Phi$ be a $(\mathbb{Z}[t], X)$-graph such that $l(\Phi)=n$ and $U(\Phi)=$ $\left\{u_{1}, \ldots, u_{n}\right\}$. By finitely many free and u-foldings, where $u \in U(\Phi)$, one can 
transform $\Phi$ into a $(\mathbb{Z}[t], X)$-graph $\Phi_{0}$ such that $\Phi_{0}(n-1)$ is $U$-folded and all $u_{n}$-components of $\Phi_{0}$ are $u_{n}$-folded.

Proof of Claim 4.14. Let $C_{1}, \ldots, C_{K}, K \in \mathbb{N}$ be the list of all $u_{n}$-components of $\Phi$. Using finitely many $u_{n}$-foldings every $C_{i}$ can be transformed into a $u_{n^{-}}$ folded $u_{n}$-component independently of all other $C_{j}, j \neq i$. Observe that after all these transformations in the resulting graph $\Phi^{\prime}$ we can have that $\Phi^{\prime}(n-1)$ is not $U$-folded any more. But this can happen only if two vertices of $\Phi(n-1)$ have been identified, that is, if performed $u_{n}$-foldings have decreased the number of vertices in the $u_{n}$-components and

$$
\sum_{i=1}^{K}\left|V\left(C_{i}\right)\right|<\sum_{i=1}^{K}\left|V\left(C_{i}^{\prime}\right)\right|,
$$

where $C_{i}^{\prime}$ is a $u_{n}$-component of $\Phi^{\prime}$ which is obtained from $C_{i}$. By the induction hypothesis we transform $\Phi^{\prime}$ into a new $(\mathbb{Z}[t], X)$-graph so that $\Phi^{\prime}(n-1)$ transforms into $\Phi^{\prime}(n-1)$ which is $U$-folded. Correspondingly, $C_{1}^{\prime}, \ldots, C_{K}^{\prime}$ transform into $C_{1}^{\prime \prime}, \ldots, C_{K}^{\prime \prime}$ and a $u_{n}$-component $C_{i}^{\prime \prime}, i \in[1, K]$ can be not $u_{n}$-folded only if two vertices of $C_{i}^{\prime}$ have been identified while transforming $\Phi^{\prime}$ into $\Phi^{\prime \prime}$. In this case again we have

$$
\sum_{i=1}^{K}\left|V\left(C_{i}^{\prime}\right)\right|<\sum_{i=1}^{K}\left|V\left(C_{i}^{\prime \prime}\right)\right| .
$$

Both inequalities above show that in a finite number of steps we obtain a $(\mathbb{Z}[t], X)$ graph $\Phi_{0}$ such that $\Phi_{0}(n-1)$ is $U$-folded and all $u_{n}$-components of $\Phi_{0}$ are $u_{n}$-folded. The claim is proved.

Claim 4.15. Let $\Phi$ be a $(\mathbb{Z}[t], X)$-graph such that $l(\Phi)=n$ and $U(\Phi)=$ $\left\{u_{1}, \ldots, u_{n}\right\}$. Adding finitely many edges to $\Phi$ and using finitely many free and $u$-foldings, where $u \in U(\Phi)$ one can transform $\Phi$ into a $(\mathbb{Z}[t], X)$-graph $\Phi_{0}$ such that

(a) $\Phi_{0}$ satisfies (1)-(3),

(b) $\Phi_{0}(n-1)$ is $U$-folded,

(c) if $v \in V(\Phi)$ and $v_{0} \in V\left(\Phi_{0}\right)$ corresponds to $v$ then $L(\Phi, v)=L\left(\Phi_{0}, v_{0}\right)$.

Proof of Claim 4.15. By Claim 4.14 we can assume $\Phi(n-1)$ to be $U$-folded and all $u_{n}$-components of $\Phi$ to be $u_{n}$-folded.

For any edge $e \in E(\Phi)$ such that $\mu(e)=u_{n}^{k_{e}}, k_{e} \in \mathbb{Z}$ we add to $\Phi$ a new path $p_{e}$ such that $o\left(p_{e}\right)=o(e), t\left(p_{e}\right)=t(e), \mu\left(p_{e}\right)=\pi\left(u_{n}\right)^{k_{e}}$. The graph thus obtained we denote by $\Phi^{\prime}$. Obviously $L(\Phi, v) \subseteq L\left(\Phi^{\prime}, v\right)$. On the other hand observe that any reduced path $p$ in $\Phi^{\prime}$ which contains at least one new edge also has a subpath $p_{e}$ such that there exists $e \in E(\Phi)$ and $o(e)=o\left(p_{e}\right), t(e)=t\left(p_{e}\right), \mu(e)=\overline{\mu\left(p_{e}\right)}$. Thus, substituting $p_{e}$ by $e$ and continuing by the induction on the number of new edges in $p$ one obtains a path $p^{\prime}$ in $\Phi$ such that $o\left(p^{\prime}\right)=o(p), t\left(p^{\prime}\right)=t(p), \mu\left(p^{\prime}\right)=\overline{\mu(p)}$. Hence, $L\left(\Phi^{\prime}, v\right) \subseteq L(\Phi, v)$. 
Using the induction hypothesis and Claim 1, we obtain a new graph $\Phi^{\prime \prime}$ such that $\Phi^{\prime \prime}(n-1)$ is $U$-folded and all its $u_{n}$-components are $u_{n}$-folded. Observe that if there exists $e \in E\left(\Phi^{\prime \prime}\right)$ such that $\mu(e)=u_{n}^{k}, k \in \mathbb{Z}$ and there exists no path $p_{e}$ such that $o\left(p_{e}\right)=o(e), t\left(p_{e}\right)=t(e), \mu\left(p_{e}\right)=\pi\left(u_{n}\right)^{k}$ then it means that transformation of $\Phi^{\prime}$ into $\Phi^{\prime \prime}$ has affected $u_{n}$-components of $\Phi^{\prime}$, that is,

$$
\sum_{i=1}^{K}\left|V\left(C_{i}^{\prime \prime}\right)\right|<\sum_{i=1}^{K}\left|V\left(C_{i}^{\prime}\right)\right|,
$$

where $C_{1}^{\prime}, \ldots, C_{K}^{\prime}, K \in \mathbb{N}$ is the list of all $u_{n}$-components of $\Phi^{\prime}$ and $C_{1}^{\prime \prime}, \ldots, C_{K}^{\prime \prime}$ are the corresponding $u_{n}$-components of $\Phi^{\prime \prime}$. Hence, continuing the operation described above we can obtain a graph with the required property and in fact we can assume $\Phi^{\prime \prime}$ to be such a graph. However, $u_{n}$-components of $\Phi^{\prime \prime}$ may be not isolated.

Let $\Theta$ be a connected component of $\Phi^{\prime}(n-1)$ which contains $C_{1}^{\prime \prime}$ and let $z \in$ $V\left(\Phi^{\prime}(n-1)\right) \cap V\left(C_{1}^{\prime \prime}\right)$. By Lemma 4.10, since $\Theta$ is finite we can find all vertices of $\Theta$ which are connected with $z$ by paths labeled by finite exponents of $u_{n}$. Denote the set of these vertices by $V_{C_{1}^{\prime \prime}}$. Suppose there exists $z^{\prime} \in V_{C_{1}^{\prime \prime}} \cap C_{j}^{\prime \prime}, j \in[1, K], j \neq 1$. Hence $C_{1}^{\prime \prime}$ and $C_{j}^{\prime \prime}$ are connected by a path $p$ such that $o(p)=z, t(p)=z^{\prime}, \mu(p)=$ $\pi\left(u_{n}\right)^{k}, k \in \mathbb{Z}$. Hence we add to $\Phi^{\prime \prime}$ an edge $e_{p}$ labeled by $u_{n}^{k}$ and reduce by this the number of $u_{n}$-components of $\Phi^{\prime \prime}$. Since this number is finite then using the considerations above we obtain a new graph $\Phi_{0}$ with the properties $(1)-(3)$. The claim is proved.

Claim 4.16. Let $\Phi_{0}$ be the $(\mathbb{Z}[t], X)$-graph obtained in Claim 4.15. Adding finitely many edges to $\Phi_{0}$ one can transform $\Phi_{0}$ into a new $(\mathbb{Z}[t], X)$-graph $\Phi_{1}$ such that

(a) $\Phi_{1}$ satisfies (1)-(3),

(b) $\Phi_{1}(n-1)$ is $U$-folded,

(c) if $v_{0} \in V\left(\Phi_{0}\right)$ and $v_{1} \in V\left(\Phi_{1}\right)$ corresponds to $v_{0}$ then $L\left(\Phi_{1}, v_{1}\right)=L\left(\Phi_{0}, v_{0}\right)$,

(d) if $C$ is a $u_{n}$-component of $\Phi_{1}, v \in V(C)$ and there exists a loop $p$ in $\Phi_{1}(n-1)$ at $v$ such that $\overline{\mu(p)}=u_{n}^{k}, k \in \mathbb{N}$ then $u_{n}^{k} \in H_{u_{n}}\left(z_{C}\right)$.

Proof of Claim 4.16. Let $C_{1}, \ldots, C_{K}, K \in \mathbb{N}$ be the list of all $u_{n}$-components of $\Phi_{0}$. Let $\Theta$ be a connected component of $\Phi_{0}(n-1)$ which contains $C_{1}$ and let $z \in V\left(\Phi_{0}(n-1)\right) \cap V\left(C_{1}\right)$. Suppose there exists a loop $p$ in $\Theta$ at $z$ such that $\overline{\mu(p)}=$ $u_{n}^{k}, k>0$ (observe that we can check this effectively in view of Lemma 4.10). Since $\Phi_{0}(n-1)$ is $U$-folded we can assume that $\mu(p)=\pi\left(u_{n}\right)^{k}$ and $k$ is minimal possible. If $u_{n}^{k} \notin H_{u_{n}}(z)$ then we add to $\Phi_{0}$ a new edge $e$ such that $o(e)=t(e)=z, \mu(e)=u_{n}^{k}$. Let $\Phi_{0}^{\prime}$ be the resulting graph. Then $V\left(\Phi_{0}^{\prime}\right)=V\left(\Phi_{0}\right)$ and it is easy to see that for any $v \in V\left(\Phi_{0}\right)$ we have $L\left(\Phi_{0}^{\prime}, v\right)=L\left(\Phi_{0}, v\right)$. Moreover, $\Phi_{0}^{\prime}$ satisfies $(1)-(3)$

Let

$$
M_{1}\left(\Phi_{0}\right)=\sum_{i=1}^{K} l_{i}
$$


where $H_{u_{n}}\left(z_{C_{i}}\right) \cap\left\langle u_{n}\right\rangle=\left\langle u_{n}^{l_{i}}\right\rangle, l_{i}>0$ and $i \in[1, K]$, and

$$
M_{2}\left(\Phi_{0}\right)=\left|\left\{C_{i} \mid H_{u_{n}}\left(z_{C_{i}}\right) \cap\left\langle u_{n}\right\rangle=\varepsilon, v_{i} \in V\left(C_{i}\right), i \in[1, K]\right\}\right| .
$$

The same characteristics can be introduced for $\Phi_{0}^{\prime}$ as well and considering ordered pairs $\left(M_{1}\left(\Phi_{0}\right), M_{2}\left(\Phi_{0}\right)\right)$ and $\left(M_{1}\left(\Phi_{0}^{\prime}\right), M_{2}\left(\Phi_{0}^{\prime}\right)\right)$ we have that

$$
\left(M_{1}\left(\Phi_{0}^{\prime}\right), M_{2}\left(\Phi_{0}^{\prime}\right)\right)<\left(M_{1}\left(\Phi_{0}\right), M_{2}\left(\Phi_{0}\right)\right)
$$

with respect to the right lexicographic order. Indeed, if $H_{u_{n}}\left(z_{C_{1}}\right) \cap\left\langle u_{n}\right\rangle=\varepsilon$ then $M_{1}\left(\Phi_{0}^{\prime}\right)<M_{1}\left(\Phi_{0}\right)$ and $\left(M_{1}\left(\Phi_{0}^{\prime}\right), M_{2}\left(\Phi_{0}^{\prime}\right)\right)<\left(M_{1}\left(\Phi_{0}\right), M_{2}\left(\Phi_{0}\right)\right)$. If $H_{u_{n}}\left(z_{C_{1}}\right) \cap$ $\left\langle u_{n}\right\rangle=\left\langle u_{n}^{l_{1}}\right\rangle, l_{1}>0$ then $M_{1}\left(\Phi_{0}^{\prime}\right)=M_{1}\left(\Phi_{0}\right)$. Let $H$ be a free abelian group associated with the $u_{n}$-component $C_{1} \cup\{e\}$ of $\Phi_{0}^{\prime}$. Then $H \cap\left\langle u_{n}\right\rangle=\left\langle u_{n}^{l_{1}^{\prime}}\right\rangle$ and obviously $l_{1}>l_{1}^{\prime}$. Hence, $M_{2}\left(\Phi_{0}^{\prime}\right)<M_{2}\left(\Phi_{0}\right)$ and $\left(M_{1}\left(\Phi_{0}^{\prime}\right), M_{2}\left(\Phi_{0}^{\prime}\right)\right)<\left(M_{1}\left(\Phi_{0}\right), M_{2}\left(\Phi_{0}\right)\right)$.

The operation described above can be applied to all $u_{n}$-components of $\Phi_{0}$ and to all their vertices which also belong to $\Phi_{0}(n-1)$. The convergence of this process follows from the fact that $M_{2}(\Psi)$ is bounded for any $(\mathbb{Z}[t], X)$-graph $\Psi$ and $M_{1}(\Psi)$ is bounded for any fixed value of $M_{2}(\Psi)$. After the process stops we obtain the required graph $\Phi_{1}$. The claim is proved.

Claim 4.17. Let $\Phi$ be a $(\mathbb{Z}[t], X)$-graph such that $l(\Phi)=n$ and $U(\Phi)=$ $\left\{u_{1}, \ldots, u_{n}\right\}$. Adding finitely many edges to $\Phi$ one can transform it into a $(\mathbb{Z}[t], X)$ graph $\Phi_{0}$ such that

(a) $\Phi_{0}$ satisfies (1)-(4),

(b) $\Phi_{0}(n-1)$ is $U$-folded,

(c) if $v \in V(\Phi)$ and $v_{0} \in V\left(\Phi_{0}\right)$ corresponds to $v$ then $L\left(\Phi_{0}, v_{0}\right)=L(\Phi, v)$.

Proof of Claim 4.17. Applying Claims 4.15 and 4.16 to $\Phi$ we obtain a new graph $\Phi^{\prime}$ which has the properties listed in Claim 4.16. It follows from the proofs of the claims above that either $\Phi^{\prime}=\Phi$ or

$$
\left(M_{1}\left(\Phi^{\prime}\right), M_{2}\left(\Phi^{\prime}\right), M_{3}\left(\Phi^{\prime}\right), M_{4}\left(\Phi^{\prime}\right)\right)<\left(M_{1}(\Phi), M_{2}(\Phi), M_{3}(\Phi), M_{4}(\Phi)\right)
$$

with respect to the right lexicographic order, where $M_{3}(\Psi)$ is the total number of vertices in $u_{n}$-components of $\Psi$ and $M_{4}(\Psi)$ is the number of $u_{n}$-components of $\Psi$.

Let $C$ be a $u_{n}$-component of $\Phi^{\prime}$. If $H_{u_{n}}\left(z_{C}\right) \cap\left\langle u_{n}\right\rangle=u_{n}^{k(C)}, k(C)>0$ then for any $v \in V(C)$ we add to $\Phi^{\prime}$ a loop $p_{v}$ such that $o\left(p_{v}\right)=t\left(p_{v}\right)=v, \mu\left(p_{v}\right)=\pi\left(u_{n}\right)^{k(C)}$.

This operation can be performed for all $u_{n}$-components of $\Phi^{\prime}$ and let $\Phi^{\prime \prime}$ be the resulting graph. It is clear that

$$
\left(M_{1}\left(\Phi^{\prime \prime}\right), M_{2}\left(\Phi^{\prime \prime}\right), M_{3}\left(\Phi^{\prime \prime}\right), M_{4}\left(\Phi^{\prime \prime}\right)\right)=\left(M_{1}\left(\Phi^{\prime}\right), M_{2}\left(\Phi^{\prime}\right), M_{3}\left(\Phi^{\prime}\right), M_{4}\left(\Phi^{\prime}\right)\right) .
$$

If $\Phi^{\prime \prime}(n-1)$ is not $U$-folded then by iterating application of Claims 4.14-4.16 eventually we obtain the graph $\Phi_{0}$ with the properties (1)-(4) such that $\Phi_{0}(n-1)$ is $U$-folded. The claim is proved. 

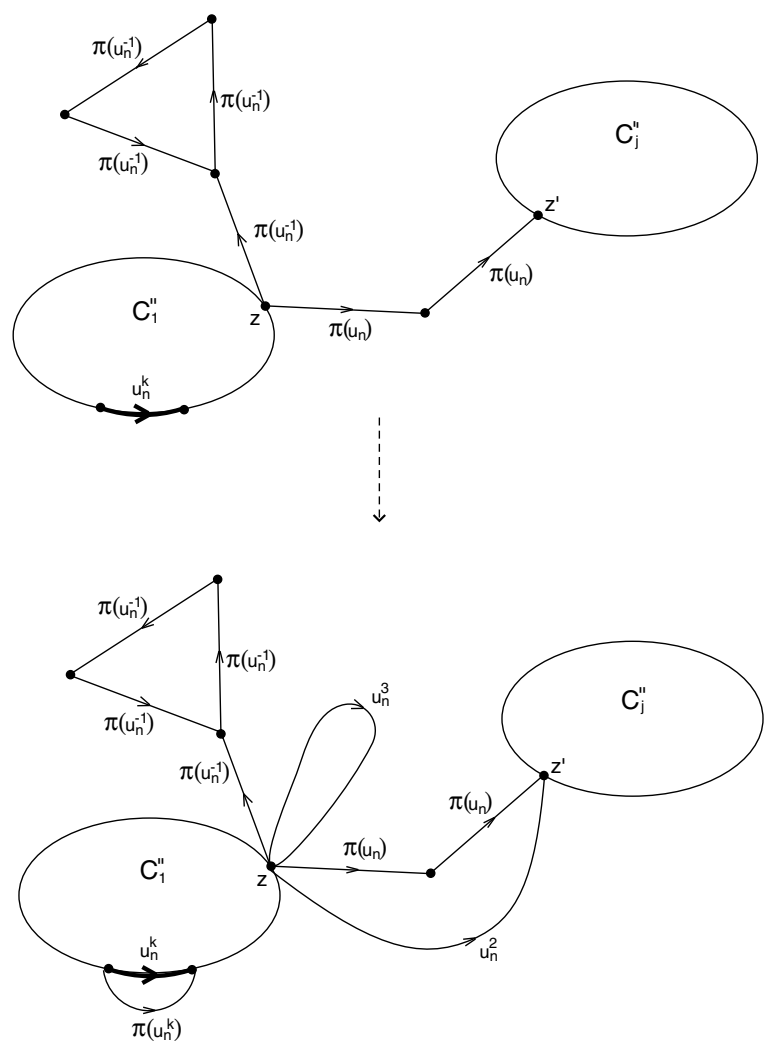

Fig. 4. Getting properties (1)-(4).

Claim 4.18. Let $\Phi$ be a $(\mathbb{Z}[t], X)$-graph such that $l(\Phi)=n$ and $U(\Phi)=$ $\left\{u_{1}, \ldots, u_{n}\right\}$. Adding and eliminating finitely many edges to $\Phi$ one can transform it into a $(\mathbb{Z}[t], X)$-graph $\Phi_{0}$ such that

(a) $\Phi_{0}$ satisfies $(1)-(5)$,

(b) $\Phi_{0}(n-1)$ is $U$-folded,

(c) if $v \in V(\Phi)$ and $v_{0} \in V\left(\Phi_{0}\right)$ corresponds to $v$ then $L\left(\Phi_{0}, v_{0}\right)=L(\Phi, v)$.

Proof of Claim 4.18. In view of Claim 4.17 we can assume that $\Phi$ satisfies (1)-(5) and $\Phi(n-1)$ is $U$-folded.

Let $C$ be a $u_{n}$-component in $\Phi$. Since $C$ is $u_{n}$-folded then by definition there exists a positively-oriented path $P_{C}$ associated with $C$ such that $V(C)=V\left(P_{C}\right)$. Let $v_{1}, v_{2} \in V(C), v_{1} \neq v_{2}$. Consider the following possibilities.

Suppose the subpath $p=e_{1} \cdots e_{k}$ of $P_{C}$ is such that $o(p)=v_{1}, t(p)=v_{2}$ contains an edge labeled by an infinite exponent of $u_{n}$, say $e_{j}$ and suppose there exists an integer $k_{p}$ such that $\overline{\mu(p)} * u_{n}^{-k_{p}} \in H_{u_{n}}\left(Z_{C}\right)$. If $H_{u_{n}}\left(z_{C}\right) \cap\left\langle u_{n}\right\rangle=\varepsilon$ then such $k_{p}$ is unique, while if $H_{u_{n}}\left(v_{1}\right) \cap\left\langle u_{n}\right\rangle \neq \varepsilon$ then there are infinitely many $r \in \mathbb{Z}$ 


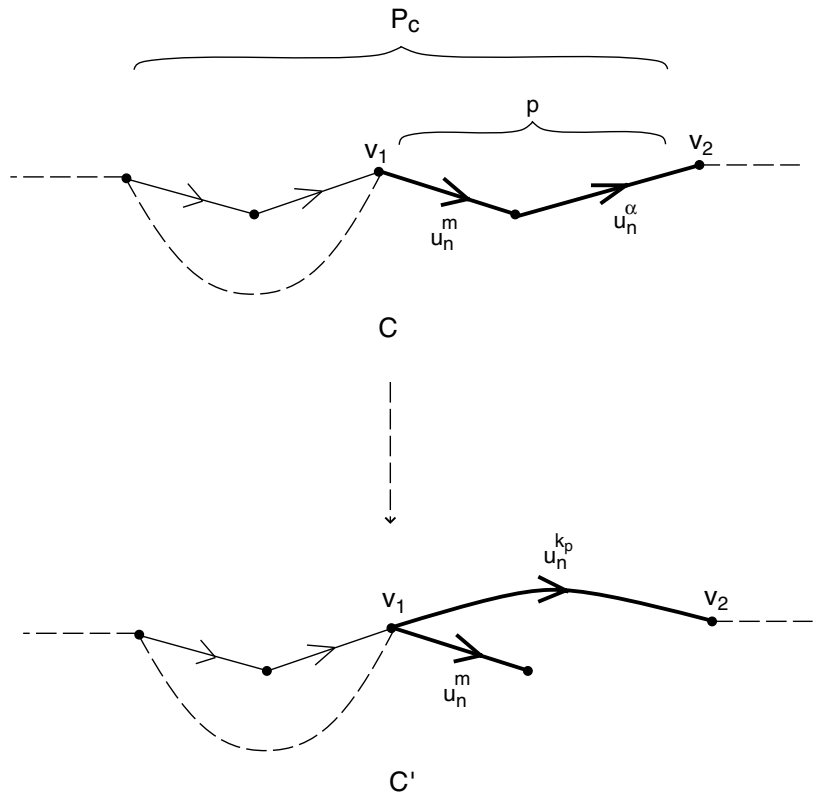

Fig. 5. Getting the property (5) $(\alpha \gg 0)$.

such that $\overline{\mu(p)} * u^{-r} \in H_{u_{n}}\left(z_{C}\right)$ all of which belong to the coset $u^{k_{p}} *\left(H_{u_{n}}\left(z_{C}\right) \cap\right.$ $\left.\left\langle u_{n}\right\rangle\right)$ and this ensures that it does not matter which one of them is chosen. Thus, we add to $C$ an edge $e$ such that $o(e)=v_{1}, t(e)=v_{2}, \mu(e)=u_{n}^{k_{p}}$ but eliminate an edge $e_{j}$ from $P_{C}$. Observe that this operation does not change $H_{u_{n}}\left(z_{C}\right)$. Moreover, if we denote the resulting graph by $\Phi^{\prime}$ then $V\left(\Phi^{\prime}\right)=V(\Phi)$ and for any $v \in V(\Phi)$ we have $L\left(\Phi^{\prime}, v\right)=L(\Phi, v)$. In addition, obviously $M_{1}\left(\Phi^{\prime}\right)=M_{1}(\Phi), M_{2}\left(\Phi^{\prime}\right)=$ $M_{2}(\Phi), M_{3}\left(\Phi^{\prime}\right)=M_{3}(\Phi), M_{4}\left(\Phi^{\prime}\right)=M_{4}(\Phi)$.

Let

$$
M_{5}(\Psi)=\sum_{i=1}^{K} m\left(C_{i}\right)
$$

where $C_{1}, \ldots, C_{K}$ are $u_{n}$-components of $\Psi$ and $m\left(C_{i}\right)$ is the number of edges labeled by infinite exponents of $u_{n}$ in $P_{C_{i}}, i \in[1, K]$. Then $\left(M_{1}\left(\Phi^{\prime}\right), M_{2}\left(\Phi^{\prime}\right), M_{3}\left(\Phi^{\prime}\right)\right.$, $\left.M_{4}\left(\Phi^{\prime}\right), M_{5}\left(\Phi^{\prime}\right)\right)<\left(M_{1}(\Phi), M_{2}(\Phi), M_{3}(\Phi), M_{4}(\Phi), M_{5}(\Phi)\right)$ with respect to the right lexicographic order. It follows that using the operation described above one can minimize the number of edges of $\Phi$ labeled by infinite exponents of $u_{n}$ in all $u_{n}$-components of $\Phi$. If necessary one can iterate the process together with the claims above to obtain the required graph $\Phi_{0}$. The claim is proved.

Claim 4.19. Let $\Phi$ be a $(\mathbb{Z}[t], X)$-graph such that $l(\Phi)=n$ and $U(\Phi)=$ $\left\{u_{1}, \ldots, u_{n}\right\}$. Adding and eliminating finitely many edges to $\Phi$ one can transform 
it into a $(\mathbb{Z}[t], X)$-graph $\Phi_{0}$ such that

(a) $\Phi_{0}$ satisfies (1)-(6),

(b) $\Phi_{0}(n-1)$ is $U$-folded,

(c) if $v \in V(\Phi)$ and $v_{0} \in V\left(\Phi_{0}\right)$ corresponds to $v$ then $L\left(\Phi_{0}, v_{0}\right)=L(\Phi, v)$.

Proof of Claim 4.19. In view of Claim 4.18 we can assume that $\Phi$ satisfies (1)-(5) and $\Phi(n-1)$ is $U$-folded.

Let $C$ be a $u_{n}$-component in $\Phi$. Since $C$ is $u_{n}$-folded then by definition there exists a positively-oriented path $P_{C}$ associated with $C$ such that $V(C)=V\left(P_{C}\right)$. Let $v_{1}, v_{2} \in V(C), v_{1} \neq v_{2}$.

Suppose there exists a reduced path $r$ in $\Phi(n-1)$ such that $o(r)=v_{1}, t(r)=v_{2}$ and $\overline{\mu(r)}=u_{n}^{l}, l>0$. Since $\Phi(n-1)$ is $U$-reduced by Proposition 4.9 we can assume $r$ to be label reduced and $\mu(r)=\pi\left(u_{n}\right)^{l}$. Then, there exists a unique label reduced path $q$ in $\Phi(n-1)$ such that $o(q)=v_{1}, t(q)=v_{2}$ and $\mu(q)=\pi\left(u_{n}\right)^{k}, l \geq k>0$ and for any two initial subpaths $q_{1}, q_{2}$ of $q$ such that $\mu\left(q_{1}\right)=\pi\left(u_{n}\right)^{k_{1}}, \mu\left(q_{2}\right)=$ $\pi\left(u_{n}\right)^{k_{2}}, k_{1}<k_{2}<k$ we have $t\left(q_{1}\right) \neq t\left(q_{2}\right)$.

Since $v_{1}, v_{2} \in V(C)$ then there exists a reduced subpath $p$ of $P_{C}$ such that $o(p)=v_{1}, t(p)=v_{2}, \overline{\mu(p)}=u_{n}^{\alpha}, \alpha \in \mathbb{Z}[t]$.

If $p$ consists only of edges labeled by finite exponents of $u_{n}$ then from the properties (3) and (4) of $\Phi$ and from the fact that $\Phi(n-1)$ is $U$-folded it follows that there exists a unique label reduced path $p^{\prime}$ in $\Phi(n-1)$ such that $o\left(p^{\prime}\right)=$ $o(p), t\left(p^{\prime}\right)=t(p), \mu\left(p^{\prime}\right)=\pi\left(u_{n}\right)^{\alpha}$. Hence, $o(q)=o\left(p^{\prime}\right), t(q)=t\left(p^{\prime}\right)$. If $p$ is positive then so is $p^{\prime}$ and we have $\mu\left(p^{\prime}\right)=\mu(q)$, otherwise we have a contradiction either with the choice of $q$ or with the fact that $\Phi(n-1)$ is $U$-folded. Thus, by Proposition 4.9 we have $q=p^{\prime}$. If $p$ is negative then $p^{\prime}$ is also negative and we have a positively oriented loop in $\Phi(n-1)$ at $v_{1}$ which is a concatenation $q p^{\prime-1}$ so $\overline{\mu\left(q p^{\prime-1}\right)} \in H_{u_{n}}\left(z_{C}\right)$ otherwise a contradiction with (4).

Thus, we can assume that $p$ contains an edge labeled by an infinite exponent of $u_{n}$. Then $\overline{\mu(p)} * \overline{\mu(q)}^{-1} \notin H_{u_{n}}\left(z_{C}\right)$ otherwise we have a contradiction with (5). So we add to $C$ an edge $e$ labeled by $u_{n}^{k}$ such that $o(e)=v_{1}, t(e)=v_{2}$. Denote the resulting graph by $\Phi^{\prime}$. Observe that after the performed operation the new $u_{n^{-}}$ component $C \cup\{e\}$ may not be $u_{n}$-folded. Applying Claim 5 to $\Phi^{\prime}$ we obtain a new graph $\Phi^{\prime \prime}$ and it follows from the proof of Claim 5 that $\left(M_{1}\left(\Phi^{\prime \prime}\right), M_{2}\left(\Phi^{\prime \prime}\right), M_{3}\left(\Phi^{\prime \prime}\right)\right.$, $\left.M_{4}\left(\Phi^{\prime \prime}\right), M_{5}\left(\Phi^{\prime \prime}\right)\right)<\left(M_{1}\left(\Phi^{\prime}\right), M_{2}\left(\Phi^{\prime}\right), M_{3}\left(\Phi^{\prime}\right), M_{4}\left(\Phi^{\prime}\right), M_{5}\left(\Phi^{\prime}\right)\right)$ with respect to the right lexicographic order. Hence, after finitely many iterations of the process described above we obtain the required graph $\Phi_{0}$. The claim is proved.

Claim 4.20. Let $\Phi$ be a $(\mathbb{Z}[t], X)$-graph such that $l(\Phi)=n$ and $U(\Phi)=$ $\left\{u_{1}, \ldots, u_{n}\right\}$. Adding and eliminating finitely many edges to $\Phi$ one can transform it into a $(\mathbb{Z}[t], X)$-graph $\Phi_{0}$ such that

(a) $\Phi_{0}$ satisfies (1)-(7),

(b) $\Phi_{0}(n-1)$ is $U$-folded,

(c) if $v \in V(\Phi)$ and $v_{0} \in V\left(\Phi_{0}\right)$ corresponds to $v$ then $L\left(\Phi_{0}, v_{0}\right)=L(\Phi, v)$. 
Proof of Claim 4.20. In view of Claim 4.19 we can assume that $\Phi$ satisfies (1)-(6) and $\Phi(n-1)$ is $U$-folded.

Let $C$ be a $u_{n}$-component in $\Phi$. Using Lemma 4.10 we find all paths labeled by $\pi\left(u_{n}\right)^{m}, m \in \mathbb{Z}$ in $\Phi(n-1)$ which originate from vertices of $C$. The endpoints of these paths compose a finite set $S$.

Let $v_{1} \in V(C)$ and suppose there exists a reduced path $r$ in $\Phi(n-1)$ for which $o(r)=v_{1}, t(r)=v_{2}, v_{2} \in S-\notin V(C)$ and $\overline{\mu(r)}=u_{n}^{l}, l \in \mathbb{Z}$. Since $\Phi(n-1)$ is $U$ reduced by Proposition 4.9 we can assume $r$ to be label reduced and $\mu(r)=\pi\left(u_{n}\right)^{l}$. Also, without loss of generality we can assume $l>0$. Then, there exists a unique label reduced path $p$ in $\Phi(n-1)$ for which we have $o(p)=v_{1}, t(p)=v_{2}$ and $\mu(p)=\pi\left(u_{n}\right)^{k}, l \geq k>0$ and such that for any two initial subpaths $p_{1}, p_{2}$ of $p$ such that $\mu\left(p_{1}\right)=\pi\left(u_{n}\right)^{m_{1}}, \mu\left(p_{2}\right)=\pi\left(u_{n}\right)^{m_{2}}, m_{1}<m_{2}<k$ we have $t\left(p_{1}\right) \neq t\left(p_{2}\right)$. Let us denote $a_{i}=t\left(p_{i}\right)$, where $p_{i}$ is an initial subpath of $p$ such that $\mu\left(p_{i}\right)=$ $\pi\left(u_{n}\right)^{i}, i \in[1, k]$.

If $u_{n}^{k} \in H_{u_{n}}\left(z_{C}\right)$ then by Proposition 4.9 there exists a unique label reduced cycle $q$ at $v_{1}$ in $\Phi(n-1)$ labeled by the generator of $H_{u_{n}}\left(z_{C}\right) \cap\left\langle u_{n}\right\rangle$. Hence $p=q$ because of the choice of $p$ and the fact that $\Phi(n-1)$ is $U$-folded. So $v_{1}=v_{2}-$ contradiction. Hence $u_{n}^{k} \notin H_{u_{n}}\left(z_{C}\right)$.

Let $v$ be any vertex in $V(C)$ and let $q_{v}=e_{1} \cdots e_{m}$ be a label reduced subpath of $P_{C}$ such that $o\left(q_{v}\right)=v, t\left(q_{v}\right)=v_{1}, \mu\left(e_{i}\right)=u_{n}^{\alpha_{i}}, i \in[1, m]\left(q_{v}\right.$ exists since $C$ is $u_{n}$-reduced).

(i) Suppose there exists $v \in V(C)$ such that $\overline{\mu\left(q_{v}\right)} * u_{n}^{k} \in H_{u_{n}}\left(z_{C}\right)$. Then all edges in $q_{v}$ are labeled by finite exponents of $u_{n}$ - otherwise (5) breaks for $\Phi$ and we have a contradiction. Finally, since $\Phi$ has properties (3) and (4) and $\Phi(n-1)$ is $U$-folded it means that $p$ connects two vertices in $C$ and, hence, $v_{2} \in C$ contradiction.

(ii) Suppose for any $v \in V(C)$ we have $\overline{\mu\left(q_{v}\right)} * u_{n}^{k} \notin H_{u_{n}}\left(z_{C}\right)$. Then we add to $C$ a path $f_{1} f_{2} \cdots f_{k}$ so that $o\left(f_{1}\right)=v_{1}, t\left(f_{i}\right)=o\left(f_{i+1}\right)=a_{i}, i \in[1, k-$ 1], $t(k)=v_{2}, \mu\left(f_{i}\right)=u_{n}, i \in[1, k]$. Applying $k u_{n}$-foldings we fold $f_{1} f_{2} \cdots f_{k}$ with $P_{C}$ and the obtained $u_{n}$-component is $u_{n}$-folded because of our assumption on $k$. If we denote resulting graph by $\Phi^{\prime}$ then $\Phi^{\prime}(n-1)$ is $U$-folded because $v_{2}$ has not been identified with any vertex of $\Phi$ and $\Phi^{\prime}$ has the properties (1)-(6). Also we have $M_{5}\left(\Phi^{\prime}\right)=M_{5}(\Phi)$, although $M_{3}\left(\Phi^{\prime}\right)=M_{3}(\Phi)+k$.

Since $S$ is finite then applying the operation described above we add to $C$ all vertices from $S$ and the same can be done for all $u_{n}$-components of $\Phi$. On each step the resulting graph has the properties (1)-(6) and its $n-1$-level is $U$-folded. In finitely many steps we get a the required graph $\Phi_{0}$. The claim is proved.

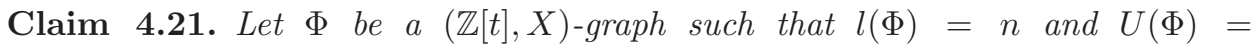
$\left\{u_{1}, \ldots, u_{n}\right\}$. Suppose $\Phi(n-1)$ is $U$-folded and $\Phi$ satisfies (1)-(7). Let $C$ be a $u_{n}$-component of $\Phi, v \in V(C)$ and let $p$ be $a\left(u_{n}, \delta\right)$-irregular path $p$ of type I originating from $v$. Then either $p$ is doubled in $\Phi(n-1)$ or there exists a path $r=r_{1} r_{2}$ in $\Phi(n-1)$ such that $o(r)=o(p), t(r)=t(p), \overline{\mu(r)}=\overline{\mu(p)}$, 


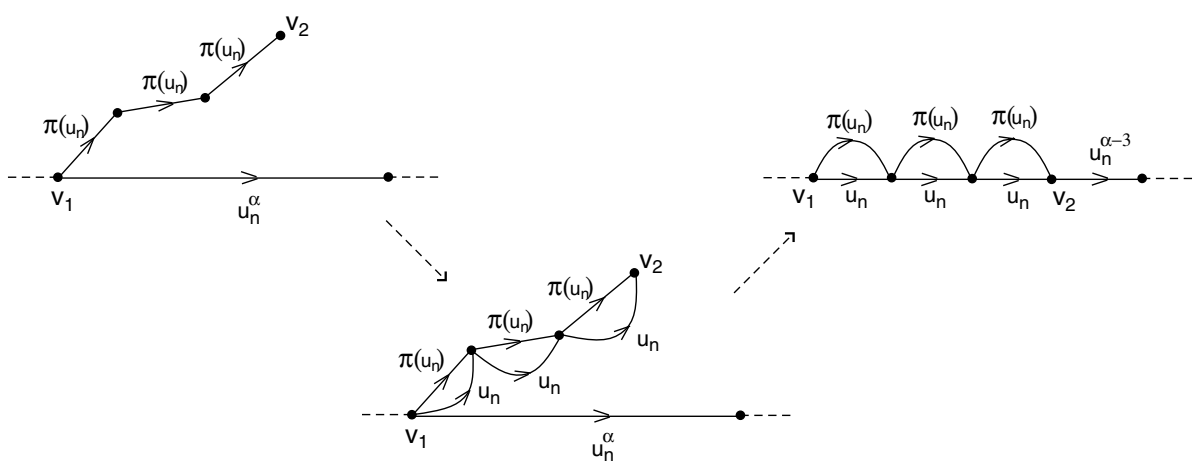

Fig. 6. Getting the property (7).

$\mu\left(r_{1}\right)=\pi\left(h_{1}\right) u_{n-1}^{\alpha_{1}} \pi\left(h_{2}\right) \cdots \pi\left(h_{m}\right), \overline{\mu\left(r_{2}\right)}=u_{n-1}^{\alpha_{m}} \circ h_{m+1} \circ c, u_{n-1}^{\gamma}=h_{m+1} \circ u^{\prime}$, where $\gamma \in\{1,-1\}, \alpha_{m} \gamma>0$ and

$$
\pi\left(u_{n}\right)^{\delta}=\pi\left(h_{1}\right) u_{n-1}^{\alpha_{1}} \pi\left(h_{2}\right) \cdots u_{n-1}^{\alpha_{m}} \pi\left(h_{m+1}\right) .
$$

Proof of Claim 4.21. Without loss of generality we can assume $\delta=1$. Let

$$
p=d_{1} p_{1} d_{2} \cdots d_{k} p_{k} d_{k+1}
$$

where $p_{i}$ is a path in some $u_{n-1}$-component of $\Phi(n-1)$ and $d_{i}$ is a path in $\Phi(n-1)$ which does not contain edges labeled by $u_{n-1}^{\alpha}, \alpha \in \mathbb{Z}[t]$. We can assume that there exists no initial subpath $d^{\prime}{ }_{i}$ of $d_{i} p_{i} \cdots d_{k} p_{k} d_{k+1}$ such that $\overline{\mu\left(d^{\prime}{ }_{i}\right)}=u_{n-1}^{ \pm 1}$ and there exists no terminal subpath $d^{\prime \prime}{ }_{i}$ of $d_{i}$ such that $\overline{\mu\left(d^{\prime \prime}{ }_{i}\right)}=u_{n-1}^{ \pm 1}$ - otherwise, since $\Phi(n-1)$ is $U$-folded, by induction we can assume that $t\left(d^{\prime}{ }_{i}\right)$ and $o\left(d^{\prime \prime}{ }_{i}\right)$ belong to some $u_{n-1}$-components and there exist edges $e^{\prime}, e^{\prime \prime}$ such that $o\left(e^{\prime}\right)=o\left(d^{\prime}{ }_{i}\right), t\left(e^{\prime}\right)=$ $t\left(d_{i}^{\prime}\right), \mu\left(e^{\prime}\right)=u_{n-1}^{ \pm 1}, o\left(e^{\prime \prime}\right)=o\left(d^{\prime \prime}{ }_{i}\right), t\left(e^{\prime \prime}\right)=t\left(d^{\prime \prime}{ }_{i}\right), \mu\left(e^{\prime \prime}\right)=u_{n-1}^{ \pm 1}$, so that we can substitute $d^{\prime}{ }_{i}, d^{\prime \prime}{ }_{i}$ by $e^{\prime}, e^{\prime \prime}$ correspondingly and consider the path $p^{\prime}$ obtained from $p$ by such substitutions. Let $\overline{\mu\left(p_{i}\right)}=u_{n-1}^{\beta_{i}}$.

Observe that by the properties of standard decompositions $h_{1}$ does not have $u_{n-1}$ as a terminal segment. On the other hand $\left|\overline{\mu\left(d_{1}\right)}\right| \geq\left|h_{1}\right|$ because of the choice of $h_{1}$.

(i) If $\left|\overline{\mu\left(d_{1}\right)}\right|=\left|h_{1}\right|$ then automatically $\overline{\mu\left(d_{1}\right)}=h_{1}$. Now, either $\beta_{1} \leq \alpha_{1}$ or $\beta_{1}>\alpha_{1}, u_{n-1}^{\gamma}=h_{2} \circ u^{\prime}, \gamma \in\{1,-1\}, u^{\prime} \neq \varepsilon$ and $\pi\left(u_{n}\right)=\pi\left(h_{1}\right) u_{n-1}^{\alpha_{1}} \pi\left(h_{2}\right)-$ otherwise a contradiction with the choice of $h_{2}$.

In the former case, if $\beta_{1}=\alpha_{1}$ then we proceed with $d_{2}$, as before with $d_{1}$, but if $\beta_{1}<\alpha_{1}$, then $\overline{\mu\left(d_{2}\right)}$ contains $u_{n-1}$ as an initial segment, that is, $d_{2}$ is $\left(u_{n-1}, 1\right)$ irregular of type I or II and by induction there exists a doubling path $z=z_{1} z_{2}$ for $d_{2}$ such that $o\left(d_{2}\right)=o(z), t\left(d_{2}\right)=t(z), \overline{\mu(z)}=\overline{\mu(p)}, \mu\left(z_{1}\right)=\pi\left(u_{n-1}\right), t\left(z_{1}\right)=o\left(z_{2}\right)$ and $t\left(p_{1}\right)$ belong to the same $u_{n-1}$-component $D$. So, we have either a path $d_{1} p_{1}$ or a path $d_{1} p_{1} z_{1}$ labeled by $\pi\left(h_{1}\right) u_{n-1}^{\alpha_{1}}$. 
In the latter case, $u_{n}=h_{1} \circ u_{n-1}^{\alpha_{1}} \circ h_{2}$ and there exists the required path $r=r_{1} r_{2}$, where $\mu\left(r_{1}\right)=\pi\left(h_{1}\right)$ and $\overline{\mu\left(r_{2}\right)}=u_{n-1}^{\alpha_{1}} \circ h_{2} \circ c, u_{n-1}^{\gamma}=h_{2} \circ u^{\prime}, \gamma \in\{1,-1\}$.

(ii) If $\left|\overline{\mu\left(d_{1}\right)}\right|>\left|h_{1}\right|$ then by [10, Lemma 6.9] we should have $\overline{\mu\left(d_{1}\right)}=h_{1} \circ$ $u_{n-1}^{k}, k \in \mathbb{Z}$, where the sign of $k$ depends on the sign of $\alpha_{1}$ and we can assume $k>0$. In fact, because of our assumption about $d_{i}$ we have $k=1$. Hence, $d_{1}$ is $\left(u_{n-1}, 1\right)$-irregular of type I or II and by induction there exists a doubling path $z=z_{1} z_{2}$ for $d_{1}$ such that $o\left(d_{1}\right)=o(z), t\left(d_{1}\right)=t(z), \mu\left(z_{2}\right)=\pi\left(u_{n-1}\right), t\left(z_{1}\right)=o\left(z_{2}\right)$ belongs to some $u_{n-1}$-component $D$. Observe that $t\left(z_{1}\right)$ and $t\left(d_{1}\right)$ belong to the same $u_{n-1}$-component $D$.

We proceed with $p_{1}$. We have either $\beta_{1}+1 \leq \alpha_{1}$ or $\beta_{1}+1>\alpha_{1}, u_{n-1}^{\gamma}=h_{2} \circ$ $u^{\prime}, \gamma \in\{1,-1\}, u^{\prime} \neq \varepsilon$ and $\pi\left(u_{n}\right)=\pi\left(h_{1}\right) u_{n-1}^{\alpha_{1}} \pi\left(h_{2}\right)$ - otherwise a contradiction with the choice of $h_{2}$.

If $\beta_{1}+1=\alpha_{1}$ then we proceed with $d_{2}$, as before with $d_{1}$. If $\beta_{1}+1<\alpha_{1}$, then $\overline{\mu\left(d_{2}\right)}$ contains $u_{n-1}$ as an initial segment, that is, $d_{2}$ is $\left(u_{n-1}, 1\right)$-irregular of type I or II and by induction there exists a doubling path $z^{\prime}=z_{1}^{\prime} z_{2}^{\prime}$ for $d_{2}$ such that $o\left(d_{2}\right)=o\left(z^{\prime}\right), t\left(d_{2}\right)=t\left(z^{\prime}\right), \mu\left(z_{1}^{\prime}\right)=\pi\left(u_{n-1}\right)$ and $t\left(z_{1}^{\prime}\right)=o\left(z_{2}^{\prime}\right), t\left(p_{1}\right)$ belong to the same $u_{n-1}$-component $D$. So, we have either a path $z_{1} z_{2} p_{1}$ or a path $z_{1} z_{2} p_{1} z_{1}^{\prime}$ labeled by $\pi\left(h_{1}\right) u_{n-1}^{\alpha_{1}}$.

If $\beta_{1}+1>\alpha_{1}, u_{n-1}^{\gamma}=h_{2} \circ u^{\prime}, \gamma \in\{1,-1\}, u^{\prime} \neq \varepsilon$ and $\pi\left(u_{n}\right)=\pi\left(h_{1}\right) u_{n-1}^{\alpha_{1}} \pi\left(h_{2}\right)$ then $u_{n}=h_{1} \circ u_{\underline{n-1}}^{\alpha_{1}} \circ h_{2}$ and there exists the required path $r=r_{1} r_{2}$, where $\mu\left(r_{1}\right)=\pi\left(h_{1}\right)$ and $\overline{\mu\left(r_{2}\right)}=u_{n-1}^{\alpha_{1}} \circ h_{2} \circ c$.

We have considered the first step and now the proof of the claim follows by induction on $m$.

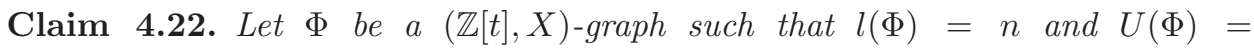
$\left\{u_{1}, \ldots, u_{n}\right\}$. Suppose $\Phi(n-1)$ is $U$-folded and $\Phi$ satisfies $(1)-(7)$. If $C$ is a $u_{n^{-}}$ component of $\Phi, v \in V(C)$ and there is a doubled $\left(u_{n}, \delta\right)$-irregular path $p$ of type I originating from $v$ then any $\left(u_{n}, \delta\right)$-irregular path $r$ of type I originating from $v$ is also doubled.

Proof of Claim 4.22. Suppose there exists an undoubled $\left(u_{n}, \delta\right)$-irregular path $r$ of type I originating from $v$. By Claim 4.21 we have $r=z_{1} z_{2}, \mu\left(z_{1}\right)=$ $\pi\left(h_{1}\right) u_{n-1}^{\alpha_{1}} \pi\left(h_{2}\right) \cdots \pi\left(h_{m}\right), \overline{\mu\left(z_{2}\right)}=u_{n-1}^{\alpha_{m}} \circ h_{m+1} \circ c(r), u_{n-1}^{\gamma}=h_{m+1} \circ u^{\prime}$, where $\gamma \in\{1,-1\}$ and

$$
\pi\left(u_{n}\right)^{\delta}=\pi\left(h_{1}\right) u_{n-1}^{\alpha_{1}} \pi\left(h_{2}\right) \cdots u_{n-1}^{\alpha_{m}} \pi\left(h_{m+1}\right) .
$$

Since $p$ is doubled then there exists a label reduced path $q=q_{1} q_{2}$ in $\Phi(n-1)$ such that $o(q)=v, t(q)=t\left(q_{2}\right)=t(p), \overline{\mu(q)}=\overline{\mu(p)}, \mu\left(q_{1}\right)=\pi\left(u_{n}\right)^{\delta}, t\left(q_{1}\right) \in V(C)$. Observe that $q_{1}=z_{1} r_{1}$, where $\mu\left(r_{1}\right)=u_{n-1}^{\alpha_{m}} \pi\left(h_{m+1}\right)$. We use the notation $r_{1}=$ $r_{2} r_{3}$, where $\mu\left(r_{2}\right)=u_{n-1}^{\alpha_{m}}$ and $\mu\left(r_{3}\right)=\pi\left(h_{m+1}\right)$.

Consider $a(r)=u_{n-1}^{\alpha_{m}} \circ h_{m+1} \circ c(r)$. Without loss of generality we can assume $\alpha_{m}>0$, so $\gamma=1$. Since $\alpha_{m} \gg 0$ it follows that

$$
\pi(a(r))=u_{n-1}^{\beta(r)} \circ a_{1} \circ \cdots \circ a_{k}
$$


and $\beta(r) \geq \alpha_{m}+\gamma . \Phi(n-1)$ is $U$-folded so there exists a path $d$ in $\Phi(n-1)$ such that $o(d)=o\left(z_{2}\right), t(d)=t\left(z_{2}\right), \mu(d)=\pi(a(r))$ and we denote by $d_{1}$ the initial subpath of $d$ such that $\mu\left(d_{1}\right)=u_{n-1}^{\beta(r)}$. Observe that $t\left(r_{2}\right) \sim_{u_{n-1}} t\left(d_{1}\right)$. Since $\Phi(n-1)$ is $U$ folded there exists a continuation $r_{3}^{\prime}$ of $r_{3}$ such that $t\left(r_{3}^{\prime}\right) \sim_{u_{n-1}} o\left(r_{3}\right) \sim_{u_{n-1}} t\left(d_{1}\right)$. Hence, there is a label reduced path $z$ such that $o(z)=t\left(r_{3}\right), t(z)=t(r)$,

$$
\overline{\mu(z)}=u^{\prime} \circ u_{n-1}^{\beta(r)-\alpha_{m}-\gamma} \circ a_{1} \circ \cdots \circ a_{k}
$$

and $q_{1} z$ is label reduced. Finally, $\overline{\mu\left(q_{1} z\right)}=\overline{\mu(r)}, o\left(q_{1} z\right)=o(r), t\left(q_{1} z\right)=t(r)$ so $q_{1} z$ is a doubling of $r$ - contradiction with our assumption. The claim is proved.

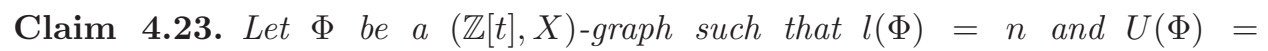
$\left\{u_{1}, \ldots, u_{n}\right\}$. Suppose $\Phi(n-1)$ is $U$-folded and $\Phi$ satisfies (1)-(7). If $C$ is a $u_{n}$-component of $\Phi, v \in V(C)$ and there are $\left(u_{n}, \delta\right)$-irregular paths $p=$ $\underline{p_{\Phi(n-1)} p_{\Phi(n)}}, r=r_{\Phi(n-1)} r_{\Phi(n)}$ of type II originating from $v$ then $\overline{\mu\left(p_{\Phi(n-1)}\right)}=$ $\overline{\mu\left(r_{\Phi(n-1)}\right)}$.

Proof of Claim 4.23. Without loss of generality we can assume $\delta=1$. We have $p=p_{\Phi(n-1)} p_{\Phi(n)}, p_{\Phi(n-1)} \in \Phi(n-1), \overline{\mu\left(p_{\Phi(n-1)}\right)}=w_{1}, \overline{\mu\left(p_{\Phi(n)}\right)}=w_{2} \circ c(p)=$ $u_{n}^{\gamma_{p}} \circ c_{1}(p), \gamma_{p} \in \mathbb{Z}[t]-\mathbb{Z}, u_{n}=w_{1} \circ w_{2}, w_{2} \neq \varepsilon$ and $r=r_{\Phi(n-1)} r_{\Phi(n)}, r_{\Phi(n-1)} \in$ $\Phi(n-1), \overline{\mu\left(r_{\Phi(n-1)}\right)}=w_{3}, \overline{\mu\left(r_{\Phi(n)}\right)}=w_{4} \circ c(r)=u_{n}^{\gamma_{r}} \circ c_{1}(r), \gamma_{r} \in \mathbb{Z}[t]-\mathbb{Z}$, $u_{n}=w_{3} \circ w_{4}, w_{4} \neq \varepsilon$.

Observe that if $\gamma_{p} \gamma_{r}>0$, that is, for example $\gamma_{p}>0, \gamma_{r}<0$ then we have $w_{3} \circ w_{4}=w_{3} \circ w_{4}^{-1}$ and $w_{4}=w_{4}^{-1}=\varepsilon-$ contradiction. So, we can assume $\gamma_{p}>0, \gamma_{r}>0$

Suppose $\left|w_{1}\right|>\left|w_{3}\right|$. Then $w_{1}=w_{3} \circ a, a \neq \varepsilon$. Observe that $w_{1} \circ u_{n}^{\gamma_{p}}$ and $w_{3} \circ u_{n}^{\gamma_{r}}$ have common initial segment of the length at least $2\left|u_{n}\right|$ and we obtain

$$
w_{1} \circ w_{3} \circ w_{4} \circ w_{3}=w_{3} \circ w_{1} \circ w_{2} \circ w_{1} .
$$

It follows that $\left[w_{1}, w_{3}\right]=\left[w_{1}, a\right]=\left[w_{3}, a\right]=\varepsilon$. On the other hand we have

$$
w_{4} \circ w_{3}=w_{2} \circ w_{1}
$$

Since $w_{4}=a \circ w_{2}$ then $a \circ w_{2} \circ w_{3}=w_{2} \circ w_{3} \circ a$ and using the fact that $w_{3} \circ a=a \circ w_{3}$ we obtain $w_{2} \circ a=a \circ w_{2}$. Hence, $\left[w_{2}, a\right]=\left[w_{2}, w_{1}\right]=\varepsilon$. It follows that $w_{1}, w_{2}$ are powers of the same element and $u_{n}$ is a power - contradiction.

Hence, $\left|w_{1}\right|=\left|w_{3}\right|$ and thus $w_{1}=w_{3}, w_{2}=w_{4}$. The claim is proved.

Claim 4.24. Let $\Phi$ be $(\mathbb{Z}[t], X)$-graph such that $U(\Phi)=n$ and $U(\Phi)=$ $\left\{u_{1}, \ldots, u_{n}\right\}$. Suppose $\Phi(n-1)$ is $U$-folded and $\Phi$ satisfies (1)-(7). If $v \in V(\Phi)$ and $p$ a path in $\Phi(n-1)$ such that $o(p)=v, \mu(p)=\pi(w)$ then for any $w_{1}, w_{2}$ such that $w=w_{1} \circ w_{2}$ one can construct $a(\mathbb{Z}[t], X)$-graph $\Phi_{0}$ such that

(a) $\Phi_{0}$ satisfies $(1)-(7)$,

(b) $\Phi(n-1)$ is $U$-folded,

(c) if $v_{1} \in V(\Phi)$ and $v_{2} \in V\left(\Phi_{0}\right)$ corresponds to $v_{1}$ then $L\left(\Phi, v_{1}\right)=L\left(\Phi_{0}, v_{2}\right)$, 
(d) there exists a path $q=q_{1} q_{2}$ in $\Phi_{0}$ such that $o(q)=v, t(q)=t(p), \mu\left(q_{1}\right)=$ $\pi\left(w_{1}\right), \mu\left(q_{2}\right)=\pi\left(w_{2}\right)$.

\section{Proof of Claim 4.24. Let}

$$
\pi(w)=\pi\left(h_{1}\right) u_{n-1}^{\alpha_{1}} \pi\left(h_{2}\right) \cdots u_{n-1}^{\alpha_{m}} \pi\left(h_{m+1}\right) .
$$

We conduct the proof by induction on $k=\max \left\{U\left(w_{1}\right)\right\}$.

Let $k=0$. Hence $w \in F(X)$. Let $r_{1} r_{2}$ be the initial subpath of $p$ such that $r_{2} \in E(\Phi), \mu\left(r_{1}\right)=\pi(g), g \in F(X), \mu\left(r_{2}\right)=u_{1}^{\alpha}, \alpha \in \mathbb{Z}[t]-\mathbb{Z}$. We can assume that $u_{1} \in F(X)$ and $\alpha>0$.

If $\left|w_{1}\right| \leq|g|$ then $\Phi$ already has the required path and we are done. Hence assume that $\left|w_{1}\right|>|g|$. It follows that $w_{1}=g \circ u_{1}^{k} \circ c$, where $c$ is an initial subpath of $u_{1}$ (possibly trivial) and $k \geq 0$.

Observe that $o\left(r_{2}\right)$ belongs to some $u_{1}$-component of $\Phi$. If $H_{u_{1}}\left(z_{D}\right) \cap\left\langle u_{1}\right\rangle \neq \varepsilon$ then we are done setting $\Phi_{0}=\Phi$. Thus, assume $H_{u_{1}}\left(z_{D}\right) \cap\left\langle u_{1}\right\rangle=\varepsilon$. If there exists a path in $D$ originating from $v$ and labeled by $u_{1}^{k+1}$ then there exists a corresponding path in $\Gamma(0)$ with the same label and endpoints - we are done in this case. So, we can assume that there is no such path in $D$. We split $r_{2}$ into $f_{1} f_{2} \cdots f_{k+2}$ so that $\mu\left(f_{i}\right)=u_{1}, i \in[1, k+1], \mu\left(f_{k+2}\right)=u_{1}^{\alpha-1}$ and add paths $z_{i}, i \in[1, k+1]$ so that $o\left(z_{i}\right)=o\left(f_{i}\right), t\left(z_{i}\right)=t\left(f_{i}\right), \mu\left(z_{i}\right)=\pi\left(u_{1}\right)$. Thus obtained graph $\Phi_{0}$ is $U$-folded and there exists a path $q=q_{1} q_{2}$ in $\Phi_{0}$ such that $o(q)=v, t(q)=t(p), \mu\left(q_{1}\right)=$ $\pi\left(w_{1}\right), \mu\left(q_{2}\right)=\pi\left(w_{2}\right)$.

We assume that we can construct the required graph $\Phi_{0}$ for any $w_{1}$ such that $k=\max \left\{U\left(w_{1}\right)\right\}=n-2$. Suppose $k=n-1$.

Let $r_{1} r_{2}$ be the initial subpath of $p$ such that $\mu\left(r_{1}\right)=\pi\left(h_{1}\right), \overline{\mu\left(r_{2}\right)}=u_{n-1}^{\alpha_{1}}$. We can assume that $\alpha_{1}>0$.

Since $\max \left\{U\left(w_{1}\right)\right\}=n-1$ it follows that $w_{1}=h_{1} \circ u_{n-1}^{\theta} \circ c, \alpha_{1} \geq$ $\theta \gg 0, u_{n-1}=c \circ c_{1}$. Observe that $o\left(r_{2}\right)$ belongs to some $u_{n-1}$-component $D$ and we can assume that there exists no path in $D$ originating from $o\left(r_{2}\right)$ and labeled by $u_{n-1}^{\theta}$.

We add an edge $e_{1}$ and a vertex $v_{1}$ to $\Phi$ so that $o\left(e_{1}\right)=o\left(r_{2}\right), t\left(e_{1}\right)=$ $v_{1}, \mu\left(e_{1}\right)=u_{n-1}^{\theta}$. Thus we have a $u_{n-1}$-component $D \cup\left\{e_{1}\right\}$ in the resulting graph. We fold $e_{1}$ with $P_{D}$ and obtain a new graph $\Phi^{\prime}$ with the $u_{n-1}$-component $D^{\prime}$ which corresponds to $D \cup\left\{e_{1}\right\}$. It is easy to see that $H_{u_{n-1}}\left(z_{D^{\prime}}\right)=H_{u_{n-1}}\left(z_{D}\right)$. By our assumption $v_{1}$ is not identified with any vertex of $D$ in $D^{\prime}$ hence $v_{1}$ divides some edge $f \in E(D)$ into two edges $f_{1}$ and $f_{2}$ so that $o\left(f_{1}\right)=o(f), t\left(f_{1}\right)=o\left(f_{2}\right), t\left(f_{2}\right)=$ $t(f), \mu(f)=u_{n-1}^{\lambda}, \mu\left(f_{1}\right)=u_{n-1}^{\lambda_{1}}, \mu\left(f_{2}\right)=u_{n-1}^{\lambda_{2}}$. Observe that $|\lambda| \gg 0$ and we can assume $\lambda$ to be positive.

$$
\text { 1. } \lambda_{1}, \lambda_{2} \gg 0 \text {. }
$$

We can assume that $D^{\prime}$ has the property (5), otherwise the situation reduces to the case when either $\lambda_{1}$ or $\lambda_{2}$ is finite. Hence, there exists no path in $D^{\prime}$ originating from $v_{1}$ and labeled by $u_{n-1}$. 
(a) $H_{u_{n-1}}\left(z_{D^{\prime}}\right) \cap\left\langle u_{n-1}\right\rangle=u_{n-1}^{k} \neq \varepsilon$.

Let $\phi$ be a graph which consists of a single loop labeled by $\pi\left(u_{n-1}\right)^{k}$. Since $l(\phi)=n-2$ then by the induction hypothesis there exists a corresponding $U$ folded graph $\phi^{\prime}$. Let $a_{i}, i \in[0, k-1]$ be the vertices of $\psi$ which correspond to the endpoints of paths in $\psi$ starting at $o(\phi)$ and labeled by $\pi\left(u_{n-1}\right)^{i}, i \in[0, k-1]$, and let $\theta=\theta_{1} \theta_{2} \cdots \theta_{k}$ be a graph which consists of a single loop such that $t\left(\theta_{i}\right)=$ $o\left(\theta_{i+1}\right), i \in[1, k-1], t\left(\theta_{k}\right)=o\left(\theta_{1}\right), \mu\left(\theta_{i}\right)=u_{n-1}, i \in[1, k]$. Then we identify $a_{i}$ with $o\left(\theta_{i+1}\right)$ for all $i \in[0, k-1]$. It easy to see that $\phi^{\prime} \cup \theta$ is $U$-folded and we denote it by $\Psi$.

Now we add $\Psi$ to $\Phi^{\prime}$ so that $a_{0}$ is identified with $v_{1}$ and fold $\theta$ with $P_{D^{\prime}}$ (in fact with $f_{2}$ ). Hence we obtain a new graph $\Phi^{\prime \prime}$ with the $u_{n-1}$-component $D^{\prime \prime}$ which corresponds to $D^{\prime}$ and such that $\Phi^{\prime \prime}(n-1)$ is $U$-folded. Observe that there exists an edge $e_{2}$ in $D^{\prime \prime}$ such that $o\left(e_{2}\right)=v_{1}, \mu\left(e_{2}\right)=u_{n-1}$ and a path $d$ in $\Phi^{\prime \prime}(n-2)$ such that $o(d)=v_{1}, t(d)=t\left(e_{2}\right), \mu(d)=\pi\left(u_{n-1}\right)$.

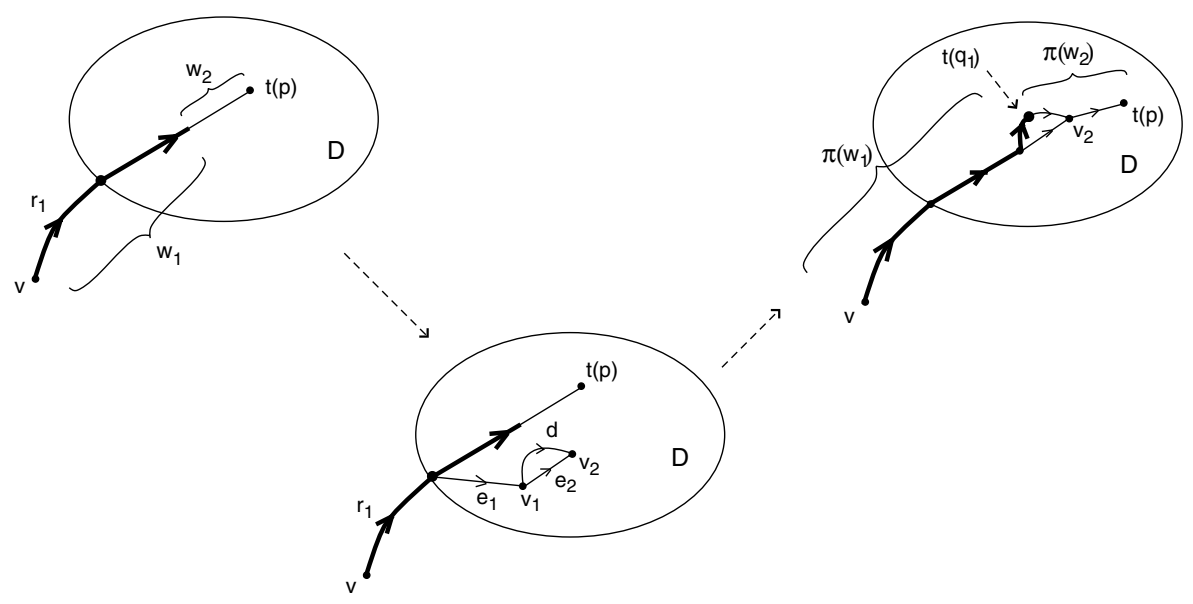

Fig. 7. Proof of Claim 4.24.

(b) $H_{u_{n-1}}\left(z_{D^{\prime}}\right) \cap\left\langle u_{n-1}\right\rangle=\varepsilon$.

We divide $f_{2}$ into two edges $e_{2}$ and $f_{2}^{\prime}$ such that $o\left(e_{2}\right)=o\left(f_{2}\right), t\left(e_{2}\right)=$ $o\left(f_{2}^{\prime}\right), t\left(f_{2}^{\prime}\right)=t\left(f_{2}\right), \mu\left(e_{2}\right)=u_{n-1}, \mu\left(f_{2}^{\prime}\right)=u_{n-1}^{\lambda_{2}-1}$. Let $d$ be a graph which consists of a single path labeled by $\pi\left(u_{n-1}\right)$. Since $l(d)=n-2$ then by the induction hypothesis there exists a corresponding $U$-folded graph $\psi$. Abusing notation, we denote the vertices corresponding to endpoints of $d$ in $\psi$ by $o(d)$ and $t(d)$. Finally we add a path $\psi$ to $\Phi^{\prime}$ so that $o(d)=o\left(e_{2}\right), t(d)=t\left(e_{2}\right)$ and obtain a new graph $\Phi^{\prime \prime}$ with the $u_{n-1}$-component $D^{\prime \prime}$ which corresponds to $D^{\prime}$ so that $\Phi^{\prime \prime}(n-1)$ is $U$-folded.

2. Either $\lambda_{1}$ or $\lambda_{2}$ is finite.

We can assume that $\lambda_{1} \in \mathbb{Z}$. Hence $H_{u_{n-1}}\left(z_{D^{\prime}}\right) \cap\left\langle u_{n-1}\right\rangle=\varepsilon$ and then there exists no path in $D^{\prime}$ originating from $v_{1}$ and labeled by $u_{n-1}$. Then we divide $f_{2}$ 
into two edges $e_{2}$ and $f_{2}^{\prime}$ such that $o\left(e_{2}\right)=o\left(f_{2}\right), t\left(e_{2}\right)=o\left(f_{2}^{\prime}\right), t\left(f_{2}^{\prime}\right)=t\left(f_{2}\right)$, $\mu\left(e_{2}\right)=u_{n-1}, \mu\left(f_{2}^{\prime}\right)=u_{n-1}^{\lambda_{2}-1}$. Similarly to $1(\mathrm{~b})$ we add to $\Phi^{\prime}(n-2)$ a $U$-folded graph corresponding to a single path $\phi_{1}$ labeled by $\pi\left(u_{n-1}\right)^{\lambda_{1}}$ so that $o\left(\phi_{1}\right)$ is identified with $o\left(f_{1}\right)$ and $t\left(\phi_{1}\right)$ is identified with $t\left(f_{1}\right)$, and in the same way we add a $U$-folded graph corresponding to a single path $\phi_{2}$ labeled by $\pi\left(u_{n-1}\right)$ so that $o\left(\phi_{2}\right)$ is identified with $o\left(e_{2}\right)$ and $t\left(\phi_{1}\right)$ is identified with $t\left(e_{2}\right)$. The obtained graph with the $u_{n-1}$-component $D^{\prime \prime}$ which corresponds to $D^{\prime}$ we denote by $\Phi^{\prime \prime}$, and it is easy to see that $\Phi^{\prime \prime}(n-1)$ is $U$-folded.

Eventually, in all cases above in the graph $\Phi^{\prime \prime}$ there exists a path $p_{1} d p_{2}$ such that $o\left(p_{1}\right)=v, t\left(p_{1}\right)=o(d)=v_{1}, t(d)=o\left(p_{2}\right), t\left(p_{2}\right)=t(p), \mu\left(p_{1}\right)=$ $\pi\left(h_{1}\right) u_{n-1}^{\theta}, \mu(d)=\pi\left(u_{n-1}\right), \mu\left(p_{2}\right)=u_{n-1}^{\alpha_{1}-\theta} \pi\left(h_{2}\right) \cdots u_{n-1}^{\alpha_{m}} \pi\left(h_{m+1}\right)$. By the induction hypothesis considering $d$ we can construct a graph $\Phi_{0}$ in which there exists a path $d_{1} d_{2}$ such that $o\left(d_{1}\right)=o(d), t\left(d_{1}\right)=o\left(d_{2}\right), t\left(d_{2}\right)=t(d), \mu\left(d_{1}\right)=$ $\pi(c), \mu\left(d_{2}\right)=\pi\left(c_{1}\right)$. Hence, setting $q_{1}=p_{1} d_{1}, q_{2}=d_{2} p_{2}$ we get the required.

Next, in the same way we consider $w_{1}=h_{1} \circ u_{n-1}^{\alpha_{1}} \circ h_{2}^{\prime}(r)$, where $h_{2}^{\prime}$ is an initial segment of $h_{2}$ and so on. In a finite number of steps we obtain the required graph $\Phi_{0}$. The claim is proved.

Claim 4.25. Let $\Phi$ be a $(\mathbb{Z}[t], X)$-graph such that $l(\Phi)=n$ and $U(\Phi)=$ $\left\{u_{1}, \ldots, u_{n}\right\}$. Suppose $\Phi(n-1)$ is $U$-folded and $\Phi$ satisfies $(1)-(7)$. If $C$ is a $u_{n}$ component of $\Phi, v \in V(C)$ and there is a $\left(u_{n}, \delta\right)$-irregular path $p$ of type I or II originating from $v$ then adding finitely many edges to $\Phi$ one can transform it into a $(\mathbb{Z}[t], X)$-graph $\Phi_{0}$ such that

(a) $\Phi_{0}$ satisfies (1)-(8),

(b) $\Phi_{0}(n-1)$ is $U$-folded,

(c) $p$ is doubled in $\Phi_{0}$,

(d) if $w \in V(\Phi)$ and $w_{0} \in V\left(\Phi_{0}\right)$ corresponds to $w$ then $L\left(\Phi_{0}, w_{0}\right)=L(\Phi, w)$.

Proof of Claim 4.25. Without loss of generality we can assume $\delta=1$. We have the following cases.

I. $p$ is of type I.

Since $u_{n}^{\delta}$ is an initial subword of $w=\overline{\mu(p)}$ then by Claim 4.24 we transform $\Phi$ into the graph $\Phi^{\prime}$ such that there exists a path $q=q_{1} q_{2}$ in $\Phi^{\prime}(n-1)$ and $o(q)=v, t(q)=t\left(q_{2}\right)=t(p), \overline{\mu(q)}=\overline{\mu(p)}, \mu\left(q_{1}\right)=\pi\left(u_{n}\right)^{\delta}, \mu\left(q_{1}\right)=$ $\pi\left(u_{n}^{-\delta} * w\right)$. Finally to make this path $q$ a doubling of $p$ we need just to attach $t\left(q_{1}\right)$ to $C$.

II. $p$ is of type II.

We have $p=p_{\Phi(n-1)} p_{\Phi(n)}, o(p) \in V(C), p_{\Phi(n-1)} \in \Phi(n-1), \overline{\mu\left(p_{\Phi(n-1)}\right)}=$ $w_{1}, \overline{\mu\left(p_{\Phi(n)}\right)}=w_{2} \circ c=u_{n}^{\gamma} \circ c_{1}, \gamma \in \mathbb{Z}[t]-\mathbb{Z}, u_{n}=w_{1} \circ w_{2}, w_{2} \neq \varepsilon, \delta \in\{1,-1\}$. That is, $t\left(p_{\Phi(n-1)}\right) \in V(D)$, where $D$ is a $u_{n}$-component of $\Phi(n)$. Without loss of generality we can assume $\gamma>0$. Since $\gamma \gg 0$ it follows that $\pi\left(u_{n}^{\gamma} \circ c_{1}\right)=u_{n-1}^{\gamma^{\prime}} \circ \pi\left(c_{2}\right)$, where $\gamma^{\prime} \geq \gamma$ and $c_{2}$ is a terminal segment of $c_{1}$. 

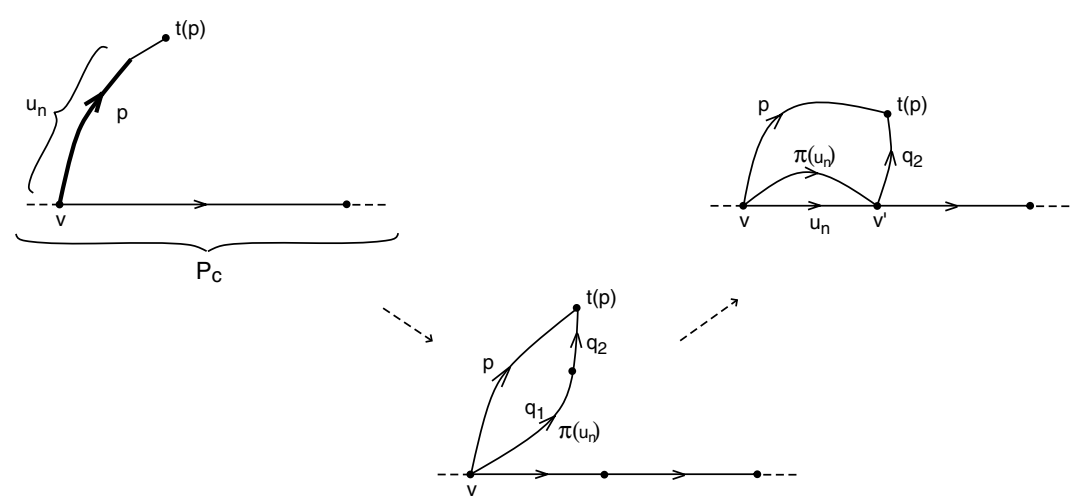

Fig. 8. Doubling of a $\left(u_{n}, 1\right)$-irregular path of type I. Possible case 1.
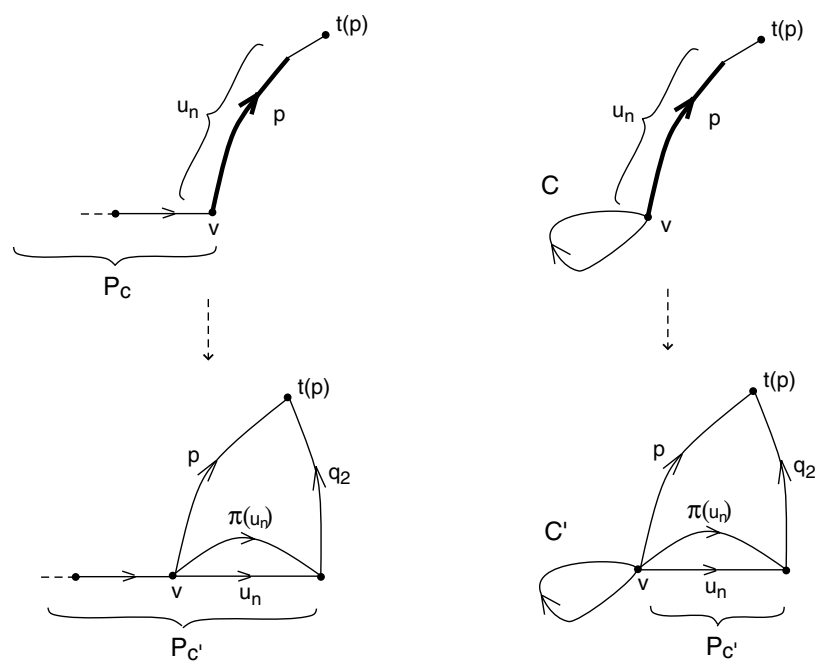

Fig. 9. Doubling of a $\left(u_{n}, 1\right)$-irregular path of type I. Possible cases 2 and 3.

1. The first edge of $p_{\Phi(n)}$ is labeled by finite exponent of $u_{n}$.

Then there exists a label reduced path $d$ labeled by $\pi\left(u_{n}\right)$ such that $o(d)=$ $t\left(p_{\Phi(n-1)}\right), t(d) \in V(D)$. On the other hand there exists a path $p^{\prime}$ such that $o\left(p^{\prime}\right)=$ $t(d), t\left(p^{\prime}\right)=t(p), \overline{\mu\left(p^{\prime}\right)}=c_{2}$, thus $p_{\Phi(n-1)} d p^{\prime}$ has the same label and endpoints as $p$. Now observe that $p_{\Phi(n-1)} d$ is $\left(u_{n}, 1\right)$-irregular of type I and the existence of its doubling impies the existence of a doubling for $p$. Hence, we reduce everything to the case I.

2. The first edge $e$ of $p_{\Phi(n)}$ is such that $o(e)=o\left(p_{\Phi(n)}\right), \mu(e)=u_{n}^{\beta}, \beta \gg 0$.

(a) $H_{u_{n}}\left(z_{D}\right) \cap\left\langle u_{n}\right\rangle \neq \varepsilon$.

By the properties (3) and (4) of $\Phi$ there exists a label reduced path $d$ labeled by $\pi\left(u_{n}\right)$ such that $o(d)=t\left(p_{\Phi(n-1)}\right), t(d) \in V(D)$. Also there exists a path $p^{\prime}$ such 
that $o\left(p^{\prime}\right)=t(d), t\left(p^{\prime}\right)=t(p), \overline{\mu\left(p^{\prime}\right)}=c_{2}$, thus $p_{\Phi(n-1)} d p^{\prime}$ has the same label and endpoints as $p$. Hence we have the reduction to the case I as in 1 .

(b) $H_{u_{n}}\left(z_{D}\right) \cap\left\langle u_{n}\right\rangle=\varepsilon$.

Observe that there exists no path in $D$ starting at $t\left(p_{\Phi(n-1)}\right)$ with the reduced label $u_{n-1}$ — otherwise $D$ is not minimal with respect to the number of infinite edges and we get a contradiction with the fact that $\Phi$ has the properties (1)-(7).

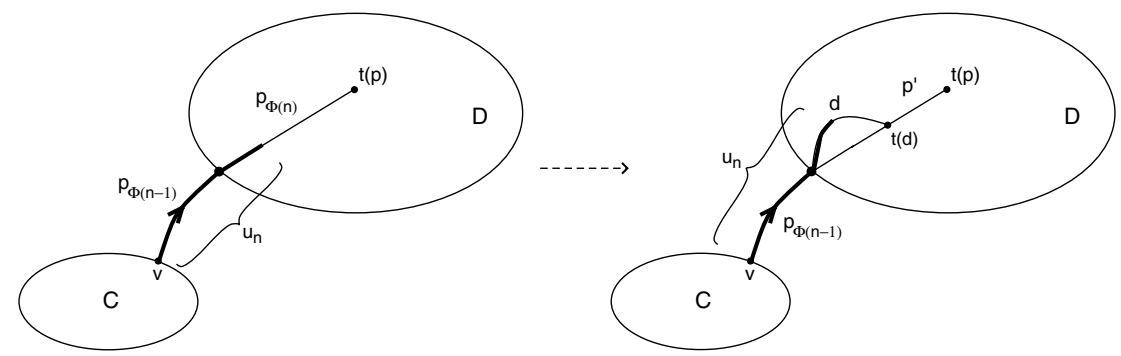

Fig. 10. Doubling of a $\left(u_{n}, 1\right)$-irregular path of type II. Reduction to I.

We divide $e$ by a new vertex $v^{\prime}$ into two edges $e_{1}$ and $e_{2}$ so that $o\left(e_{1}\right)=$ $o(e), t\left(e_{1}\right)=v^{\prime}, o\left(e_{2}\right)=v^{\prime}, t\left(e_{2}\right)=t(e), \mu\left(e_{1}\right)=u_{n}, \mu\left(e_{2}\right)=u_{n}^{\beta-1}$. The resulting graph we denote by $\Phi^{\prime}$ and the $u_{n}$-component corresponding to $D$ by $D^{\prime}$. Observe that $D^{\prime}$ is $u_{n}$-folded because of our assumption. Finally, we add a $U$-folded graph corresponding to a single path $d$ labeled by $\pi\left(u_{n}\right)$ so that $o(d)$ is identified with $o\left(e_{1}\right)$ and $t(d)$ is identified with $t\left(e_{1}\right)$. The resulting graph $\Phi^{\prime \prime}$ has the properties $(1)-(7)$ and $\Phi^{\prime \prime}(n-1)$ is $U$-folded. Finally, since $t(d) \in V\left(D^{\prime}\right)$ then there exists a path $p^{\prime}$ such that $o\left(p^{\prime}\right)=t(d), t\left(p^{\prime}\right)=t(p), \overline{\mu\left(p^{\prime}\right)}=c_{2}$, thus $p_{\Phi(n-1)} d p^{\prime}$ has the same label and endpoints as $p$. Everything reduces to the case I again.

This completes the proof of the claim.

Claim 4.26. Let $\Phi$ be a $(\mathbb{Z}[t], X)$-graph such that $l(\Phi)=n$ and $U(\Phi)=$ $\left\{u_{1}, \ldots, u_{n}\right\}$. Suppose $\Phi(n-1)$ is $U$-folded and $\Phi$ satisfies $(1)-(7)$. If $C$ is a $u_{n^{-}}$ component of $\Phi, v \in V(C)$ then adding finitely many edges to $\Phi$ one can transform it into a $(\mathbb{Z}[t], X)$-graph $\Phi_{0}$ such that

(a) $\Phi_{0}$ satisfies (1)-(8),

(b) $\Phi_{0}(n-1)$ is $U$-folded,

(c) all $\left(u_{n}, \delta\right)$-irregular paths of type III originating from $v$ are doubled in $\Phi_{0}$,

(d) if $w \in V(\Phi)$ and $w_{0} \in V\left(\Phi_{0}\right)$ corresponds to $w$ then $L\left(\Phi_{0}, w_{0}\right)=L(\Phi, w)$.

Proof of Claim 4.26. If there is no $\left(u_{n}, \delta\right)$-irregular path of type III originating from $v$ then we are done setting $\Phi_{0}=\Phi$.

Suppose on the contrary that there exists a path $p$ in $\Phi$ such that $o(p)=v, \overline{\mu(p)}=w_{1}(p), u_{n}^{\delta}=w_{1}(p) \circ w_{2}(p), w_{2}(p) \neq \varepsilon, \delta \in\{1,-1\}$ and there exists a path $p^{\prime}$ in $C$ such that $o\left(p^{\prime}\right)=o(p), \overline{\mu\left(p^{\prime}\right)}=u_{n}^{\gamma}, \gamma \in \mathbb{Z}[t], \gamma \delta>0$. 
Without loss of generality we can assume $\delta=1$, hence $\gamma>0$.

Observe that if $\gamma \in \mathbb{Z}$ then the first edge of $p^{\prime}$ is labeled by a finite exponent of $u_{n}$ because of the properties (1)-(8) of $\Phi$. In this case it follows immediately that all $\left(u_{n}, 1\right)$-irregular paths of type III originating from $v$ are doubled. This happens also when $H_{u_{n}}\left(z_{C}\right) \cap\left\langle u_{n}\right\rangle \neq \varepsilon$ or there exists an edge $f$ in $C$ such that $o(f)=v$ and $\mu(f)=u_{n}^{k}, k \in \mathbb{Z}$. Hence we can assume that $H_{u_{n}}\left(z_{C}\right) \cap\left\langle u_{n}\right\rangle=\varepsilon$ and that the first edge $e$ of $p^{\prime}$ belongs to $P_{C}$ and is labeled by an infinite positive exponent of $u_{n}$, that is, $o(e)=v, \mu(e)=u^{\beta}, \beta \gg 0$.

Observe that there exists no path in $C$ starting at $t\left(p_{\Phi(n-1)}\right)$ with the reduced label $u_{n-1}$ - otherwise $C$ is not minimal with respect to the number of infinite edges and we get a contradiction with the fact that $\Phi$ has the properties (1)-(7).

We divide $e$ by a new vertex $v^{\prime}$ into two edges $e_{1}$ and $e_{2}$ so that $o\left(e_{1}\right)=$ $o(e), t\left(e_{1}\right)=v^{\prime}, o\left(e_{2}\right)=v^{\prime}, t\left(e_{2}\right)=t(e), \mu\left(e_{1}\right)=u_{n}, \mu\left(e_{2}\right)=u_{n}^{\beta-1}$. The resulting graph we denote $\Phi^{\prime}$ and the resulting $u_{n}$-component by $C^{\prime}$. Hence $C^{\prime}$ is $u_{n}$-folded.

Let $d$ be a graph which consists of a single path labeled by $\pi\left(u_{n}\right)$. Since $l(d)=$ $n-1$ then by the induction hypothesis there exists a corresponding $U$-folded graph $\psi$. Abusing notation we denote the vertices corresponding to endpoints of $d$ in $\psi$ by $o(d)$ and $t(d)$. Finally we add a path $\psi$ to $\Phi^{\prime}$ so that $o(d)=o\left(e_{1}\right), t(d)=t\left(e_{1}\right)$ and obtain a new graph $\Phi^{\prime \prime}$ with the $u_{n-1}$-component $C^{\prime \prime}$ which corresponds to $C^{\prime}$.

Observe that $\Phi^{\prime \prime}(n-1)$ is not $U$-folded since there is at least one $\left(u_{n}, 1\right)$-irregular path $p$ of type III originating from $v=o(d)$.

Let $\mathcal{P}$ be the set of all $\left(u_{n}, 1\right)$-irregular paths in $\Phi$ of type III which originate from $v$. Since $\Phi(n-1)$ is $U$-folded then by Proposition 4.9 for each $r \in \mathcal{P}$ there exists a path $p_{r}$ in $\Phi$ such that $o\left(p_{r}\right)=o(r), t\left(p_{r}\right)=t(r), \mu\left(p_{r}\right)=\overline{\mu(r)}$. So, let

$$
\mathcal{P}^{\prime}=\left\{p_{r} \mid r \in \mathcal{P}\right\} .
$$

It is easy to see that $\mathcal{P}^{\prime} \subseteq \mathcal{P}$ and $\mathcal{P}^{\prime} \neq \emptyset$. Observe that it is enough to construct doublings only for paths from $\mathcal{P}^{\prime}$.

We say that $r \in \mathcal{P}^{\prime}$ has level $k$ if $k$ is the minimal natural number such that $r \in \Phi(k)$. Hence $\mathcal{P}^{\prime}$ has a natural level structure, that is, all its elements are ranged according to their levels. Observe that levels of paths from $\mathcal{P}^{\prime}$ are bounded by $n-1$.

Below we introduce the inductive procedure which transforms $\Phi^{\prime \prime}(n-1)$ into a $U$-folded graph $\Phi_{0}$. Hence all elements of $\mathcal{P}^{\prime}$ will be doubled in $\Phi_{0}$.

Let

$$
\pi\left(u_{n}\right)=\pi\left(h_{1}\right) u_{n-1}^{\alpha_{1}} \pi\left(h_{2}\right) \cdots u_{n-1}^{\alpha_{m}} \pi\left(h_{m+1}\right)
$$

Let $k=0$.

All paths in $\mathcal{P}^{\prime}$ of level 1 are labeled by reduced words in $X^{ \pm}$. Let $p_{1} f$ be the initial subpath of $d$ such that $\mu\left(p_{1}\right)=\pi(g), g \in F(X), \mu(f)=u_{1}^{\alpha}, \alpha \in \mathbb{Z}[t]-\mathbb{Z}$. We can assume that $u_{1} \in F(X)$ and $\alpha>0$.

(i) Suppose there exist only finitely many elements of $\mathcal{P}^{\prime}$ of level 1 . Then let $r \in \mathcal{P}^{\prime}$ be the path of level 1 with the maximal length. Hence we have $\overline{\mu(r)}=$ $g \circ u_{1}^{k(r)} \circ c(r)$, where $c(r)$ is an initial segment of $u_{1}$ and all other paths from $\mathcal{P}^{\prime}$ 
of level 1 are initial subpaths of $r$. We split $f$ as $f_{1} f_{2} \cdots f_{k(r)+2}$ so that $\mu\left(f_{i}\right)=$ $u_{1}, i \in[1, k(r)+1], \mu\left(f_{k(r)+2}\right)=u_{1}^{\alpha-1}$ and add to $d$ paths $z_{i}, i \in[1, k(r)+1]$ so that $o\left(z_{i}\right)=o\left(f_{i}\right), t\left(z_{i}\right)=t\left(f_{i}\right), \mu\left(z_{i}\right)=\pi\left(u_{1}\right)$. Finally, we fold $r$ with $z_{1} z_{2} \cdots z_{k(r)+1}$.

(ii) Suppose there exist infinitely many elements of $\mathcal{P}^{\prime}$ of level 1 . Since $\Phi$ is finite it follows that for any path $r$ of level 1 we have $\overline{\mu(r)}=g \circ u_{1}^{i(r)+l(r) K} \circ c(r)$, where $i(r) \in[0, K-1], K \in \mathbb{N}, l(r) \in \mathbb{N}$ and $c(r)$ is an initial segment of $u_{1}$. Hence we add at $o(f)$ a loop $f_{1} f_{2} \cdots f_{K}$ such that $t\left(f_{i}\right)=o\left(f_{i+1}\right), i \in[1, K-$ 1], $t\left(f_{K}\right)=o\left(f_{1}\right)=o(f), \mu\left(f_{i}\right)=u_{1}, i \in[1, K]$ and a path $z_{1} z_{2} \cdots z_{K}$ such that $o\left(z_{i}\right)=o\left(z_{i}\right), t\left(z_{i}\right)=t\left(z_{i}\right), i \in[1, K], \mu\left(z_{i}\right)=\pi\left(u_{1}\right), i \in[1, K]$. Finally we fold $f_{1} f_{2} \cdots f_{K}$ with $f$ and it is easy to see that now all paths from $\mathcal{P}^{\prime}$ of level 1 can be folded with $z_{1} z_{2} \cdots z_{K}$.

We can assume that all elements of $\mathcal{P}^{\prime}$ of level $n-2$ have been folded with $d$. Suppose $k=n-1$.

Let $p_{1} f$ be the initial subpath of $d$ such that $\mu\left(p_{1}\right)=\pi\left(h_{1}\right), \mu(f)=u_{n-1}^{\alpha_{1}}$. We can assume that $\alpha_{1}>0$.

We can assume that there exists a path $r \in \mathcal{P}^{\prime}$ of level $n-1$ such that $\overline{\mu(r)}=$ $h_{1} \circ u_{n-1}^{\theta(r)}, \alpha_{1} \geq \theta(r) \gg 0$. Otherwise we are done by the induction hypothesis. Hence, $r=r_{1} r_{2}$, where $\mu\left(r_{1}\right)=\pi\left(h_{1}\right), \overline{\mu\left(r_{2}\right)}=u_{n-1}^{\theta}$ and $t\left(r_{1}\right)$ belongs to some $u_{n-1}$-component $D$ of $\Phi$. On the other hand, observe that for any path $r^{\prime} \in \mathcal{P}^{\prime}$ of level $n-1$ such that $\overline{\mu\left(r^{\prime}\right)}=h_{1} \circ u_{n-1}^{\theta\left(r^{\prime}\right)}, \alpha_{1} \geq \theta\left(r^{\prime}\right) \gg 0$ we have $r^{\prime}=r_{1}^{\prime} r_{2}^{\prime}$, where $\mu\left(r_{1}^{\prime}\right)=\pi\left(h_{1}\right), \overline{\mu\left(r_{2}^{\prime}\right)}=u_{n-1}^{\theta\left(r^{\prime}\right)}$ and $t\left(r_{1}^{\prime}\right)$ belongs to $D$. Hence we fold $f$ with $P_{D}$ and the resulting $u_{n-1}$-component $D^{\prime}$ is $u_{n-1}$-folded.

Next, we consider elements $r$ of $\mathcal{P}^{\prime}$ of level $n-2$ such that $\overline{\mu(r)}=h_{1} \circ u_{n-1}^{\alpha_{1}} \circ$ $h_{2}^{\prime}(r)$, where $h_{2}^{\prime}$ is an initial segment of $h_{2}$. In this case $r=r_{1} r_{2} r_{3}$, where $\mu\left(r_{1}\right)=$ $\pi\left(h_{1}\right), \overline{\mu\left(r_{2}\right)}=u_{n-1}^{\alpha_{1}}, \mu\left(r_{3}\right)=\pi\left(h_{2}^{\prime}\right)$ and we can assume that $r_{1} r_{2}$ is already folded with $d$. But to fold $r_{3}$ we can use the induction hypothesis and so on. In a finite number of steps we obtain the required graph $\Phi_{0}$. The claim is proved.

Claim 4.27. Let $\Phi$ be a $(\mathbb{Z}[t], X)$-graph such that $l(\Phi)=n$ and $U(\Phi)=$ $\left\{u_{1}, \ldots, u_{n}\right\}$. Adding finitely many edges to $\Phi$ one can transform it into a $(\mathbb{Z}[t], X)$ graph $\Phi_{0}$ such that

(a) $\Phi_{0}$ satisfies (1)-(8),

(b) if $v \in V(\Phi)$ and $v_{0} \in V\left(\Phi_{0}\right)$ corresponds to $v$ then $L\left(\Phi_{0}, v_{0}\right)=L(\Phi, v)$.

Proof of Claim 4.27. In view of Claim 4.20 we can assume that $\Phi$ satisfies (1)-(7) and $\Phi(n-1)$ is $U$-folded.

Let $C$ be a $u_{n}$-component of $\Phi$ and $v \in V(C)$. In view of Claim 4.25 we can construct a doubling for any $\left(u_{n}, \delta\right)$-irregular path of type I or II originating from $v$. Hence by Claims 4.22 and 4.23 all $\left(u_{n}, \delta\right)$-irregular paths of type I or II originating from $v$ are doubled too. At the same time, in view of Claim 4.26 we obtain a new graph $\Phi^{\prime}$ for which all $\left(u_{n}, \delta\right)$-irregular paths of any type originating from $v$ are doubled. Finally, since the number of $u_{n}$-components is finite as well as the number 
of vertices in them then after finitely many steps we obtain the required graph $\Phi_{0}$. The claim is proved.

By Claim 4.27, $\Gamma$ can be transformed into a $U$-folded graph $\Delta$.

It can be seen easily from the Proposition 4.13 that properties of $U$-folded graphs are similar to the properties of folded $X$-graphs introduced in [4]. In [4] $X$-graphs are used for studying the structure of subgroups in free groups and for solving various algorithmic and combinatorial problems for free groups and their subgroups. In the next subsection we try to do the same for finitely generated subgroups of $F^{\mathbb{Z}[t]}$ using ( $\left.\mathbb{Z}[t], X\right)$-graphs.

\subsection{Membership problem for subgroups of $F^{\mathbb{Z}[t]}$}

In Sec. 4.1 (see Lemma 4.2) we saw that any finite $(\mathbb{Z}[t], X)$-graph defines a subgroup in $F^{\mathbb{Z}[t]}$. We will show now that the converse statement is also true.

Proposition 4.28. Let $H$ be a finitely generated subgroup of $F^{\mathbb{Z}[t]}$. Then there exists a $U$-folded $(\mathbb{Z}[t], X)$-graph $\Gamma$ and a vertex $v$ of $\Gamma$ such that $L(\Gamma, v)=H$.

Proof. Since $H$ is finitely generated then there are elements $h_{1}, \ldots, h_{k}$ in $F^{\mathbb{Z}[t]}$ which generate $H$. Since $F^{\mathbb{Z}[t]}$ is a union of the following infinite chain of groups:

$$
F(X)=G_{0}<G_{1}<\cdots<G_{n}<\cdots,
$$

where $G_{i+1}$ is obtained from $G_{i}$ by extension of all cyclic centralizers in $G_{i}$, there exists a minimal natural number $n$ such that $h_{i} \in G_{n+1}$ for all $i \in[1, k]$.

We define an $(\mathbb{Z}[t], X)$-graph $\Gamma_{1}$ in the following way. $\Gamma_{1}$ is a wedge of $k$ circles wedged at a vertex denoted $v_{1}$. The $i$ th circle is subdivided into $n_{i}$ edges which are oriented and labeled by letters from

$$
B=X^{ \pm} \cup\left\{u^{\alpha} \mid u \in U, \alpha \in \mathbb{Z}[t]-\mathbb{Z}\right\}
$$

so that the label of the $i$ th circle (as read from $v_{1}$ to $v_{1}$ ) is precisely the word $\pi\left(h_{i}\right)$. Note that $\Gamma_{1}$ is connected.

For any cycle $p$ at $v_{1}$ in $\Gamma_{1}$ we have $\overline{\mu(p)} \in L\left(\Gamma_{1}, v_{1}\right)$ by definition. So $H \subset$ $L\left(\Gamma_{1}, v_{1}\right)$. The converse is obviously true - if $g \in L\left(\Gamma_{1}, v_{1}\right)$ then it can be realized as a reduced label of some cycle at $v_{1}$ in $\Gamma_{1}$, so it can be obtained as a finite product of basic cycles labeled by $\pi\left(h_{i}\right)$. Thus we have $H=\left\langle h_{1}, \ldots, h_{k}\right\rangle \subset L\left(\Gamma_{1}, v_{1}\right)$.

By Proposition 4.13, from $\Gamma_{1}$ by finitely many free and $u$-foldings, where $u \in$ $\bigcup_{i=1}^{k} U\left(h_{i}\right)$ one can obtain a finite $U$-folded $(\mathbb{Z}[t], X)$-graph $\Gamma$ which is connected and there exists some vertex $v$ in $\Gamma$ which corresponds to $v_{1}$ in $\Gamma_{1}$. By Lemma 4.3 we have $L\left(\Gamma_{1}, v_{1}\right)=L(\Gamma, v)$.

Observe that $\Gamma$ constructed in the proposition above is not unique in general. But all graphs associated with $H$ define the same language which coincides with $H$. 
Proposition 4.13 only states the existence of $(\mathbb{Z}[t], X)$-graph $\Gamma$ for $H$. But, in fact, the following result follows directly from the procedures described in the proofs of Lemmas 3.15, 3.17 and 3.18 and Proposition 4.13.

Proposition 4.29. There is an algorithm which, given finitely many standard decompositions of elements $h_{1}, \ldots, h_{k}$ from $F^{\mathbb{Z}[t]}$, constructs a $U$-folded $(\mathbb{Z}[t], X)$ graph $\Gamma$, such that $L(\Gamma, v)=\left\langle h_{1}, \ldots, h_{k}\right\rangle$.

The next result is a solution of membership problem for finitely generated subgroups of $F^{\mathbb{Z}[t]}$.

Proposition 4.30. Every finitely generated subgroup of $F^{\mathbb{Z}[t]}$ has a solvable membership problem. That is, there exists an algorithm which, given finitely many standard decompositions of elements $g, h_{1}, \ldots, h_{k}$ from $F^{\mathbb{Z}[t]}$, decides whether or not $g$ belongs to the subgroup $H=\left\langle h_{1}, \ldots, h_{n}\right\rangle$ of $F^{\mathbb{Z}[t]}$.

Proof. We construct a $U$-folded $(\mathbb{Z}[t], X)$-graph $\Gamma$, such that $L(\Gamma, v)=\left\langle h_{1}, \ldots, h_{k}\right\rangle$ which is a finite algorithmic procedure by Proposition 4.29.

Let

$$
F<H_{1}<H_{2}<\cdots<H_{n}
$$

be the extension series for $g$, where $g \in H_{n}$ and $H_{i+1}$ is obtained from $H_{i}$ by a centralizer extension of a single element $u_{i}$ and let $U(g)=\left\{u_{1}, \ldots, u_{n}\right\}$. Then we check if $g \in L(\Gamma, v)$ using inductive argument based on $|U(g)|$.

$g \in H=L(\Gamma, v)$ if and only if there exists a reduced cycle $p$ at $v$ in $\Gamma$ such that $g=\overline{\mu(p)}$. By Proposition 4.9 of $\Gamma$ we can assume that $\mu(p)=\pi(g)$.

Let

$$
\pi(g)=\pi\left(h_{1}\right) u_{n}^{\alpha_{1}} \pi\left(h_{2}\right) \cdots u_{n}^{\alpha_{m}} \pi\left(h_{m+1}\right) .
$$

If $|U(g)|=0$, that is, $\pi(g)$ is a reduced word in $\left\{X \cup X^{-1}\right\}$ then we just try to "read" $\pi(g)$ in $\Gamma(0)$ starting at the vertex $v$ — this can be done as shown in [4, Proposition 7.2].

Suppose there is an algorithm which "reads" a standard decomposition of an element $h \in H_{n-1}$, that is $|U(g)|<n$, starting from any point $v^{\prime} \in V(\Gamma)$ and returns answer "yes" if there exists a path in $\Gamma$ corresponding to $\pi(h)$ or "no" if such path does not exist. Then we apply this algorithm to $v$ and $\pi\left(h_{1}\right)$. If we get "no" as a result then it means by Proposition 4.9 that there exists no path for $h_{1}$ starting at $v$ in $\Gamma$ and we stop - $g$ does not belong to $H$. If we get "yes" as a result, that is, we have a path $p_{1}$ for $\pi\left(h_{1}\right)$ and if $t\left(p_{1}\right)$ belongs to some $u_{n}$-component $C$ of $\Gamma$ then we try to "read" $u_{n}^{\alpha_{1}}$ as follows. A pair $\left(P_{C}, H_{u_{n}}\left(t\left(p_{1}\right)\right)\right)$ is associated with $C$, where $P_{C}$ is a finite positively oriented path and $H_{u_{n}}\left(t\left(p_{1}\right)\right)$ is a finitely generated free abelian group. By Lemma 3.18 it is enough to check if $u_{n}^{\alpha_{1}} \in \overline{\mu(q)} * H_{u_{n}}\left(t\left(p_{1}\right)\right)$, where $q$ is a reduced subpath of $P_{C}$. Hence, if we can find such $q$ then it is unique and we proceed with $h_{2}$. If there exists no such subpath of $P_{C}$, we stop $-g$ does 
not belong to $H$. In finitely many steps we either find out that $g \notin H$ on some intermediate step or construct a path $p$ from $v$ to some $v_{1} \in V(\Gamma)$, which is labeled by $\pi(g)$. If we manage to find $p$ then we check if $v=v_{1}$ which holds if and only if $g \in H$.

\section{References}

[1] H. Bass, Covering theory for graphs of groups, J. Pure Appl. Algebra 89(1-2) (1993) $3-47$.

[2] M. Bestvina and M. Feighn, Bounding the complexity of simplicial group actions on trees, Invent. Math. 103(3) (1991) 449-469.

[3] M. J. Dunwoody, Folding sequences, The Epstein Birthday Schrift, pp. 139-158; Geom. Topol. Monogr. 1, Geom. Topol. (Coventry, 1998).

[4] I. Kapovich and A. Myasnikov, Stallings foldings and subgroups of free groups, J. Algebra 248 (2002) 608-668.

[5] I. Kapovich, A. Myasnikov and R. Weidmann, Foldings, graphs of groups and the membership problem, Int. J. Algebra Comput. 15(1) (2005) 95-128.

[6] O. Kharlampovich and A. Myasnikov, Irreducible affine varieties over a free group II: Systems in triangular quasi-quadratic form and description of residually free groups, J. Algebra 200(2) (1998) 517-570.

[7] R. Lyndon, Groups with parametric exponents, Trans. Amer. Math. Soc. 9(6) (1960) $518-533$.

[8] K. A. Mihailova, The occurrence problem for direct products of groups, Dokl. Akad. Nauk SSSR 119 (1958) 1103-1105.

[9] A. Myasnikov and V. Remeslennikov, Exponential groups II: extensions of centralizers and tensor completion of CSA-groups, Int. J. Algebra Comput. 6(6) (1996) $687-711$.

[10] A. Myasnikov, V. Remeslennikov and D. Serbin, Regular free length functions on Lyndon's free $\mathbb{Z}[t]$-group $F^{\mathbb{Z}[t]}$, in Computational and Experimental Group Theory, ed. A. Borovik, Contemp. Math. 378 (Amer. Math. Soc., 2005), pp. 37-77.

[11] J.-P. Serre, Trees (Translated from the French by John Stillwell) (Springer-Verlag, Berlin-New York, 1980).

[12] J. R. Stallings, Topology of finite graphs, Invent. Math. 71(3) (1983) 551-565.

[13] J.-R. Stallings, Foldings of $G$-trees, Arboreal Group Theory (Berkeley, CA, 1988) pp. 355-368, Math. Sci. Res. Inst. Publ. 19 (Springer, New York, 1991). 